\title{
Approximate Capacity of a Class of Gaussian Interference-Relay Networks
}

\author{
Soheil Mohajer, Member, IEEE, Suhas N. Diggavi, Member, IEEE, Christina Fragouli, Member, IEEE, and
} David N. C. Tse, Fellow, IEEE

\begin{abstract}
In this paper, we study a Gaussian relay-interference network, in which relay (helper) nodes are to facilitate competing information flows between different source-destination pairs. We focus on two-stage relay-interference networks where there are weak cross links, causing the networks to behave like a chain of $Z$ Gaussian channels. Our main result is an approximate characterization of the capacity region for such $Z Z$ and $Z S$ networks. We propose a new interference management scheme, termed interference neutralization, which is implemented using structured lattice codes. This scheme allows for over-the-air interference removal, without the transmitters having complete access the interfering signals. This scheme in conjunction a new network decomposition technique provides the approximate characterization. Our analysis of these Gaussian networks is based on insights gained from an exact characterization of the corresponding linear deterministic model.
\end{abstract}

Index Terms-Deterministic model, Gaussian wireless network, interference neutralization, lattice codes, relay-interference network, structured codes.

\section{INTRODUCTION}

$\mathbf{T}$ HE multicommodity flow problem, where multiple independent unicast sessions need to share network resources, can be solved efficiently over graphs using linear programming techniques [1]. This is not the case for wireless networks, where the broadcast and superposition nature of the wireless medium introduces complex signal interactions between the competing flows. The simplest example is the one-hop interference channel [2], where two transmitters with independent messages are attempting to communicate with their respective receivers over the wireless transmission medium. Even

Manuscript received May 01, 2010; revised October 15, 2010; accepted January 12, 2011. Date of current version April 20, 2011. S. Mohajer and C. Fragouli were supported in part by the ERC Starting Investigator Grant 240317. D. N. C. Tse was supported in part by the U.S. NSF under grant CCF-0830796. The material in this paper was presented in part at the IEEE International Symposium on Information Theory (ISIT), Seoul, South Korea, July 2009.

S. Mohajer was with the Ecole Polytechnique Fédérale de Lausanne (EPFL), Lausanne CH 1015, Switzerland. He is now with the Department of Electrical Engineering, Princeton University, Princeton, NJ 08544 USA (e-mail: smohajer@princeton.edu).

S. N. Diggavi was with the Ecole Polytechnique Fédérale de Lausanne (EPFL), Lausanne CH 1015, Switzerland. He is now with the Department of Electrical Engineering, University of California, Los Angeles, CA 90095 USA (e-mail: suhas@ee.ucla.edu).

C. Fragouli is with the School of Computer and Communication Sciences, Ecole Polytechnique Fédérale de Lausanne (EPFL), Lausanne CH 1015, Switzerland (e-mail: christina.fragouli@epfl.ch).

D. N. C. Tse is with the Department of Electrical Engineering and Computer Sciences, University of California, 257 Cory Hall, Berkeley, CA 94720 USA (e-mail: dtse@eecs.berkeley.edu).

Communicated by H. El Gamal, Associate Editor for the special issue on "Interference Networks".

Color versions of one or more of the figures in this paper are available online at http://ieeexplore.ieee.org.

Digital Object Identifier 10.1109/TIT.2011.2120210 for this simple one-hop network, the information-theoretic characterization has been open for several decades. To study more general networks, there is a clear need to understand and develop sophisticated interference management techniques.

Recently, a new approach on the capacity characterization of wireless networks has been initiated. This approach asks for $a p$ proximate instead of exact characterization. The first such result is the capacity of the two user Gaussian interference channel to within one bit by Etkin, Tse, and Wang [3]. This characterization involves new upper bounds for the capacity of the network and showing that the Han-Kobayashi scheme [2] performs close to these upper bounds. More recently, Avestimehr, Diggavi, and Tse considered relay networks in [4], wherein a message has to be transmitted from one transmitter to a (set of) receiver(s) over a network with an arbitrary network topology and number of nodes. Characterization of the capacity of such network has been an open problem, even for very simple cases since 1979 [5]. The authors introduced a deterministic model for the approximation of wireless network. This model allows the analysis to focus on signal interactions rather than the randomness of the noise. They first derived a complete characterization for the capacity of deterministic relay networks, analogous to the max-flow min-cut theorem in wired networks. Then the insight obtained by this characterization is used to devise a new relaying strategy called quantize-map-and-forward for Gaussian relay networks. This scheme, unlike all the schemes previously proposed in the literature, was demonstrated to be within a gap from the cut-set upper bound which does not depend on the channel parameters.

Building on this progress, a natural next step is to study the approximate capacity region of small-scale interference-relay networks, where there are potentially multiple hops from the sources to destinations through cooperating relays. Studying even simple two-hop topologies could help develop techniques and build insight that would enable a (perhaps approximate) characterization of capacity for more general networks. We are interested in our work in a universal type of approximation, in that it should characterize the capacity to within a constant number of bits, independently of the signal-to-noise ratio and the channel parameter values.

The focus of this paper is to study the two-stage relay-interference network illustrated in Fig. 1. In particular, we give an approximate characterization of the capacity region for special cases of these networks when some of the cross-links are weak. These are illustrated in Figs. 3(a) and 4(a), which we refer to as the ZS and ZZ Gaussian models. These networks are formed by concatenation of two layers of Z (S) networks. A Z network is able to model a common situation in a wireless network, that is when two base stations communicate to two user nodes, one 


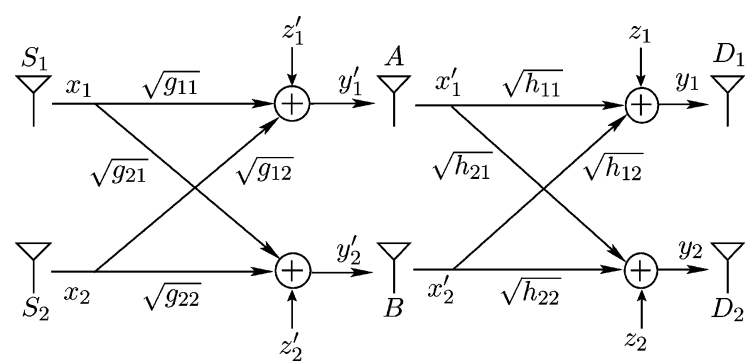

Fig. 1. Two-stage relay-interference network.

located close to one of the base-station, and the other one is on the boundary of the coverage areas of two base-stations. The $\mathrm{ZZ}$ network occurs for example when these user nodes act as relays; it could also occur when the user nodes are replaced by dedicated relay stations which have a similar connectivity. We first study a deterministic version of these problems by using the linear deterministic model introduced in [6]. An exact capacity region characterization in the deterministic case is then translated into a universally approximate characterization for the (noisy) Gaussian network. In particular, for ZS and ZZ networks we have a capacity region characterization within 2 bits (or less), independent of the operating signal-to-noise ratio and the channel parameters. The outer bound as well as the encoding schemes proposed for the Gaussian networks are inspired by the analysis of the deterministic networks. We will discuss it later in detail in Sections IV and V, where we illustrate the relaying strategies used for the deterministic networks through some examples, and then give an outline for the encoding scheme proposed for the Gaussian network.

The main tools used in literature for achievability are the Han-Kobayashi coding scheme, which includes "interference suppression" (i.e., decoding part of the interference and peeling it away) and message splitting with appropriate power allocations. Another known technique for interference management is interference alignment. In this work, we propose a new interference management technique, ${ }^{1}$ called "interference neutralization", which in the Gaussian interference-relay network requires the use of lattices to properly allow "over-the-air" cancellation of parts of the interference, without the relays decoding the interference. We do use the deterministic framework to get insight into the problem, but the deterministic network solution is new in this paper and leads to both new coding schemes as well as outer bounds. The deterministic approach also suggests a network "decomposition" approach to the achievability, which we appropriately translate to the Gaussian case through message/rate splitting. We also develop a new form of genie aided outer bounds for the interference-relay network. These outer bounds are in fact tighter than the information theoretic cut-set bounds. These new ideas enable us to obtain an approximate characterization to the networks studied in this paper, within a universal constant.

\footnotetext{
${ }^{1}$ A noise nulling technique is proposed in [7] to mitigate correlated noise in an amplify-forward relaying strategy for a single unicast "diamond" parallel relay network. However, the difference in our technique is that we use the structure of the codebooks (without necessarily decoding information) to neutralize interference, and not noise statistics. Moreover the multiple-unicast nature of the problem necessitates strategic partitioning and rate-splitting of different components of the messages.
}

A way to interpret the achievability results for the ZS networks is that the relays perform a partial-decoding of strategically split messages from the sources, and then cooperate to deliver the required messages to the destination, again through strategically splitting the messages. The power allocated to each of the sub-messages is determined using the insight derived from the deterministic model, that messages that are not intended be decoded arrive at the noise-level. The achievability for the ZZ network is slightly more sophisticated, in the sense that one of the relays is required to only decode a function of the sub-messages. The function is chosen such that its signal in combination with the transmission of the other relay causes the unwanted interference to be canceled (neutralized) at the destination. This interference neutralization is enabled in the Gaussian channel using the group property of a structured lattice code.

Work in the literature over the past decade has examined scaling laws for multiple independent flows over wireless networks, see for example [8]-[10]. The goal there is to characterize the order of the wireless network capacity as the network size grows. In contrast, in our work, instead of seeking order arguments and scaling laws, we try to characterize the capacity (perhaps within a universal constant of a few bits) for specific topologies. The interference channel is a special case of such networks, where there is only one-hop communication between the sources and destinations. There has been a surge of recent work on this topic including cooperating destinations [11] and use of feedback in inducing cooperation at the transmitters [12]. The deterministic approach developed in [6] has been successfully applied to the interference channel in [13]. The fundamental role of interference alignment in $K$-user interference channel (still a one-hop network) has been demonstrated in [14], [15].

The paper is organized as follows. Section II introduces our notation and the basic network models we study. The main results are given in Section III. Section IV illustrates the transmission techniques used in this paper through simple deterministic examples. These examples are followed by the proof outline for the Gaussian networks in Section V. The achievability and converse for the deterministic ZS network is given in Section VI, and many of these ideas are translated into the precise proof for the corresponding Gaussian ZS network in Appendix A. Section VII follows a similar program for the ZZ network, by first identifying the capacity region for the deterministic version. This allows illustration of ideas such as interference neutralization, as well as genie-aided outer bounding techniques. The precise translation of these results into Gaussian $\mathrm{ZZ}$ networks is given in Appendix B. Section VIII concludes the paper with a short discussion.

\section{Problem Statement}

A well accepted model for wireless communication is a linear Gaussian model. In this, the received signal $y_{i}[t]$ at time $t$ and node $i$, is related to the transmitted signals $\left\{x_{j}[t]\right\}$ as

$$
y_{i}[t]=\sum_{j} h_{i j} x_{j}[t]+z_{i}[t]
$$


where $z_{i}(t)$ is i.i.d. (unit-variance) Gaussian noise, and $h_{i j}$ represents the fading channel from transmitter $j$ to receiver $i$.

\section{A. Deterministic Model}

In [6], a deterministic model was proposed, to capture the essence of wireless interaction described in (1). The advantage of the deterministic model is its simplicity, which allows exact characterizations; its purpose is to build insights for the noisy wireless network in (1). The deterministic model of [6] simplifies the wireless interaction model by eliminating the noise and discretizing the channel gains through a binary expansion of $q$ bits. Therefore, the received signal $Y_{i}$, which is a binary vector of size $q$, is modeled as

$$
Y_{i}[t]=\sum_{j} N_{i j} X_{j}[t]
$$

where $N_{i j}$ is a $q \times q$ binary matrix representing the (discretized) channel transformation between nodes $j$ and $i$ and $X_{j}$ is a $q \times 1$ vector that contains the (discretized) transmitted signal. We will drop the time index $t$ when it does not play a role for simplicity. All operations in (2) are done over the binary field, $\mathbb{F}_{2}$. We use the terminology deterministic wireless network when the signal interaction model is governed by (2). The model in (2) is an approximate representation of a Gaussian fading channel, which attempts to capture the attenuation effect of the signal caused by the channel gain. This can be interpreted as the number of significant bits of a binary representation of the input, $x_{j}$, that is above the noise level. More precisely, typically the model in (2) assigns $N_{i j}=\mathbf{J}^{q-n_{i j}}$, where $\mathbf{J}$ is a shift matrix, i.e.,

$$
\mathbf{J}=\left(\begin{array}{ccccc}
0 & 0 & 0 & \cdots & 0 \\
1 & 0 & 0 & \cdots & 0 \\
0 & 1 & 0 & \cdots & 0 \\
\vdots & \ddots & \ddots & \ddots & \ddots \\
0 & \cdots & 0 & 1 & 0
\end{array}\right)_{q \times q}
$$

For real channel gain $h_{i j}$ in the Gaussian model (1), we calculate $n_{i j}$ as $n_{i j}=\left\lceil\frac{1}{2} \log \left|h_{i j}\right|^{2}\right\rceil$. The parameter $q$ is chosen such that $q \geq \max _{i, j}\left\lceil\frac{1}{2} \log \left|h_{i j}\right|^{2}\right\rceil$.

An example of a deterministic network is illustrated in Fig. 2. Each node contains several channel inputs and outputs, which are called sub-node or level through out this paper. Source $S_{1}$ can only send one bit to node $A$ and no bit to node $B$; source $S_{2}$ can send its two MSB to both $A$ and $B$, and its LSB to node $B$. The transmitted bits from nodes $S_{1}$ and $S_{2}$ interfere on the LSB that node $A$ receives.

In a deterministic network, given a cut that separates nodes $\mathcal{U}$ from node $\mathcal{V}$, the cut-value equals the rank of the transfer matrix between the nodes in $\mathcal{U}$ and $\mathcal{V}$. For example, in Fig. 2, the cut that separates nodes $\mathcal{U}=\left\{S_{1}, S_{2}, A, B\right\}$ and $\mathcal{V}=\left\{D_{1}, D_{2}\right\}$ equals

$$
\operatorname{rank}\left(\begin{array}{cccccc}
0 & 0 & 0 & 0 & 0 & 0 \\
0 & 0 & 0 & 0 & 0 & 0 \\
1 & 0 & 0 & 0 & 0 & 0 \\
1 & 0 & 0 & 0 & 0 & 0 \\
0 & 1 & 0 & 0 & 0 & 0 \\
0 & 0 & 1 & 1 & 0 & 0
\end{array}\right)=3
$$

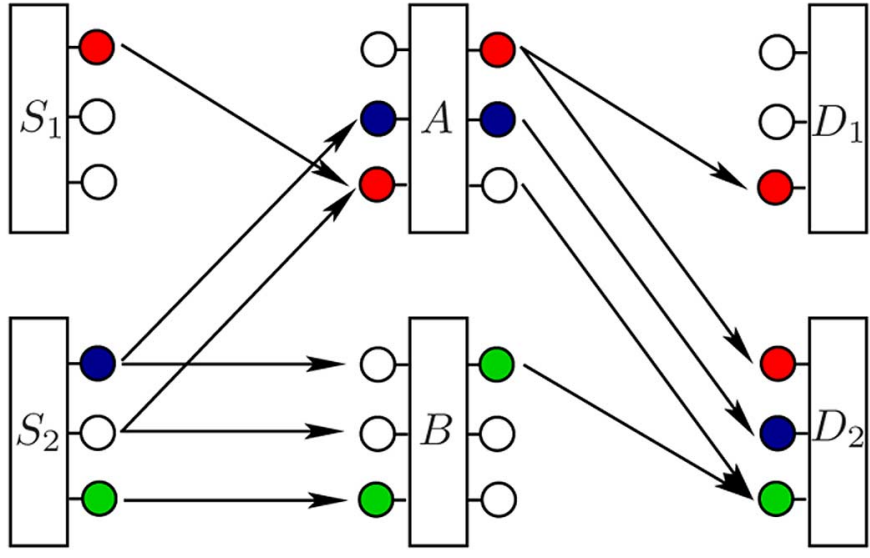

Fig. 2. Deterministic network.

The rows of this transfer matrix correspond to the six transmitted inputs by nodes $A$ and $B$, while the columns to the six receives outputs at nodes $D_{1}$ and $D_{2}$.

\section{B. Interference-Relay Network Model}

Our goal in this paper is to derive approximate capacity characterizations for a class of 2-user relay-interference networks shown in Fig. 1, which we call the XX network. We start by describing our notation for Gaussian channels.

Two transmitters, $S_{1}$ and $S_{2}$, encode their messages $W_{1}$ and $W_{2}$ of rates $R_{1}$ and $R_{2}$, respectively, and broadcast the obtained signals to the relay nodes, $A$ and $B$. Denote the transmitted signals by $x_{1}$ and $x_{2}$, and the received signals at the relays by $y_{1}^{\prime}$ and $y_{2}^{\prime}$. Then

$$
\begin{aligned}
& y_{1}^{\prime}[t]=\sqrt{g_{11}} x_{1}[t]+\sqrt{g_{12}} x_{2}[t]+z_{1}^{\prime}[t] \\
& y_{2}^{\prime}[t]=\sqrt{g_{21}} x_{1}[t]+\sqrt{g_{22}} x_{2}[t]+z_{2}^{\prime}[t]
\end{aligned}
$$

where $z_{1}^{\prime}, z_{2}^{\prime}$ are unit-variance Gaussian noises, independent of each other and of $x_{1}, x_{2}$.

The relay nodes perform any (causal) processing on their received signal sequences $\left\{y_{1}^{\prime}[t]\right\}$ and $\left\{y_{2}^{\prime}[t]\right\}$ respectively, to obtain their transmitting signal sequences, $\left\{x_{1}^{\prime}[t]\right\}$ and $\left\{x_{2}^{\prime}[t]\right\}$. The received signals at the destination nodes can be written as

$$
\begin{aligned}
& y_{1}[t]=\sqrt{h_{11}} x_{1}^{\prime}[t]+\sqrt{h_{12}} x_{2}^{\prime}[t]+z_{1}[t] \\
& y_{2}[t]=\sqrt{h_{21}} x_{1}^{\prime}[t]+\sqrt{h_{22}} x_{2}^{\prime}[t]+z_{2}[t]
\end{aligned}
$$

where $z_{1}$ and $z_{2}$ are independent zero-mean unit-variance noises, which are also independent of $x_{1}$ and $x_{2}$. There is a power constraint for each transmitted signal, that is, $\mathbb{E}\left[x_{1}^{2}\right] \leq 1$, $\mathbb{E}\left[x_{2}^{2}\right] \leq 1, \mathbb{E}\left[x_{1}^{\prime 2}\right] \leq 1$ and $\mathbb{E}\left[x_{2}^{\prime 2}\right] \leq 1$.

Each destination node $D_{i}, i=1,2$, is interested in decoding its message $W_{i}$, using its received signals $\left\{y_{i}[t]\right\}$. We define a rate pair $\left(R_{1}, R_{2}\right)$ to be admissible if there exist a transmission scheme under which $D_{1}$ and $D_{2}$ can decode $W_{1}$ and $W_{2}$, respectively, with arbitrary small (average) error probability in the standard manner [16]. This would allow two end-to-end reliable unicast sessions at rates $\left(R_{1}, R_{2}\right)$ for the source/destination pairs $\left(S_{1}, D_{1}\right)$ and $\left(S_{2}, D_{2}\right)$. 
A useful tool to examine the network problem defined above is to study its deterministic version, based on the model developed in (2). Using the deterministic approach, we can rewrite (5)-(6) as

$$
\begin{aligned}
& Y_{1}^{\prime}[t]=M_{11} X_{1}[t]+M_{12} X_{2}[t] \\
& Y_{2}^{\prime}[t]=M_{21} X_{1}[t]+M_{22} X_{2}[t]
\end{aligned}
$$

and

$$
\begin{aligned}
& Y_{1}[t]=N_{11} X_{1}^{\prime}[t]+N_{12} X_{2}^{\prime}[t] \\
& Y_{2}[t]=N_{21} X_{1}^{\prime}[t]+N_{22} X_{2}^{\prime}[t]
\end{aligned}
$$

where the matrices $\left\{M_{i j}\right\}$ and $\left\{N_{i j}\right\}$ approximately model the channels in (5)-(6), i.e., $M_{i j}=\mathbf{J}^{q-m_{i j}}, N_{i j}=\mathbf{J}^{q-n_{i j}}$. The matrix $\mathbf{J}$ is defined as in (3), while $m_{i j}=\left\lceil\frac{1}{2} \log \left|g_{i j}\right|^{2}\right\rceil$ and $n_{i j}=\left\lceil\frac{1}{2} \log \left|h_{i j}\right|^{2}\right\rceil$.

It is worth mentioning that though this network looks like cascaded interference channels, there is an important difference. Unlike the interference channel, the messages sent by the relays at the second layer of transmission need not independent, i.e., we can try to induce cooperation at the relays to transmit information to the final destinations. This distinction makes this network more interesting than a simple cascade of interference channels. In this paper, we focus on two specific realizations of the network, namely, the ZS and the ZZ networks, which further simplify the connectivity models of (5)-(6). We describe these two networks in the following, and give an approximate characterization of their admissible rate region in Section III.

Notation Alert: Throughout this paper, we use the lowercase letters $x$ and $y$ for the signals transmitted by the sources and received signals at the destinations in the Gaussian networks. The received and transmitting signals by the relays are denoted by $y^{\prime}$ and $x^{\prime}$, respectively. Similarly, uppercase letters will be used for the deterministic networks.

\section{ZS Network}

The ZS network is a special case of the interference-relay network defined in (5)-(6). In the ZS network one cross link in each layer has a negligible gain and, therefore, does not cause interference, as illustrated in Fig. 3(a). In particular, we assume $g_{21}=h_{12}=0$ in the Gaussian network, and $m_{21}=n_{12}=0$ in the deterministic network. The resulting Gaussian ZS network is shown in Fig. 3(a), and the deterministic model for this network is given in Fig. 3(b).

\section{ZZ Network}

The ZZ network is another special configuration interferencerelay network, wherein one cross link in each layer has zero gain. However, the difference is that, here the missing links are in parallel. In particular, we assume $g_{21}=h_{21}=0$ and $m_{21}=n_{21}=0$ in the Gaussian and deterministic networks, respectively. The Gaussian and corresponding deterministic ZZ networks are shown in Fig. 4.

\section{MAIN RESUlts}

In this section we present the main results of this paper, which is the approximate capacity characterization of the Gaussian ZS

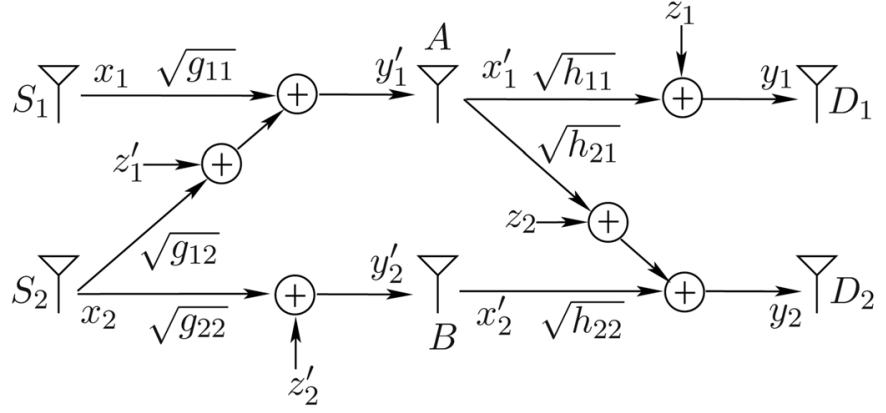

(a)

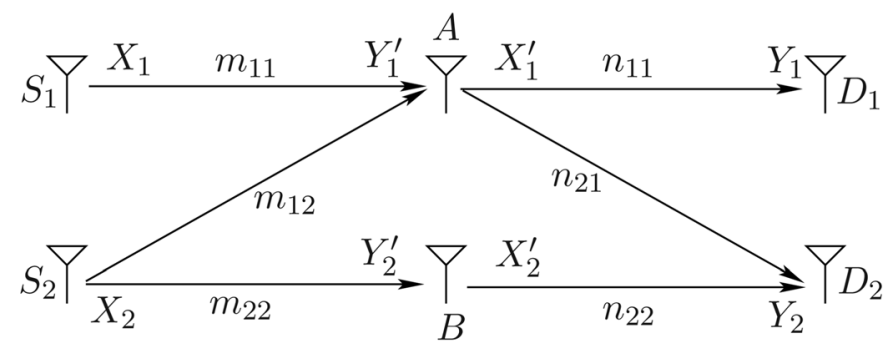

(b)

Fig. 3. ZS network. (a) The Gaussian ZS network. (b) The deterministic ZS network.

and ZZ interference-relay networks. In order to obtain such an approximate characterization, we have a complete characterization of the deterministic versions of the ZS and ZZ networks. The coding strategies for the Gaussian problems are outlined in Section V. The detailed analysis of these strategies and the corresponding outer bounds which lead to Theorems 2 and 4 are given in Appendices A and B, respectively. Most of the insights are obtained by analyzing the deterministic versions of these problems, and the exact characterizations are summarized in Theorems 1 and 3, respectively. We prove these results in Sections VI and VII, respectively. The achievability and outer bound results for the Gaussian cases are directly inspired by these results.

\section{A. ZS Network}

The ZS deterministic network illustrated in Fig. 3(b) and the corresponding Gaussian ZS network is given in Fig. 3(a). Theorems 1 and 2 give the exact and approximate (within 2 bits) characterizations of their capacity regions.

Theorem 1 (Capacity Region of Deterministic ZS Network): The capacity region of the deterministic ZS network is specified by $\mathcal{R}^{\mathrm{DZS}}$, where $\mathcal{R}^{\mathrm{DZS}}$ is the set of all rate pairs $\left(R_{1}, R_{2}\right)$ that satisfy

$$
\begin{aligned}
R_{1} & \leq m_{11} \\
R_{2} & \leq \max \left(m_{12}, m_{22}\right), \\
R_{1}+R_{2} & \leq \max \left(m_{11}, m_{12}\right)+\left(m_{22}-m_{12}\right)^{+}
\end{aligned}
$$

$R_{2} \leq m_{12}+n_{22}$,

(DZS-4) 


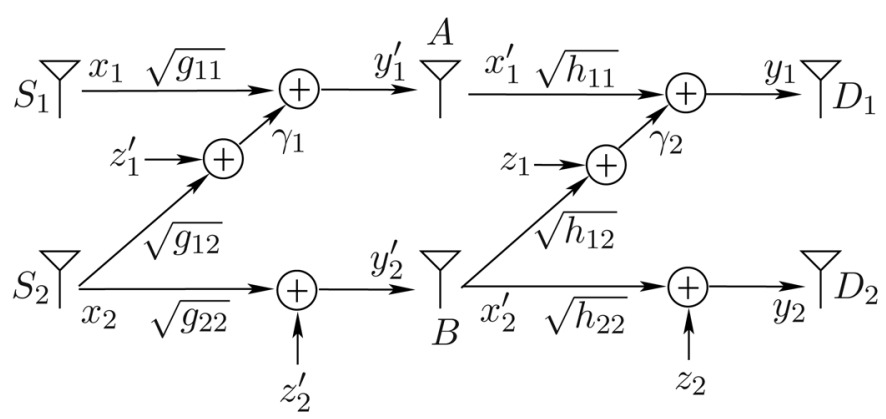

(a)

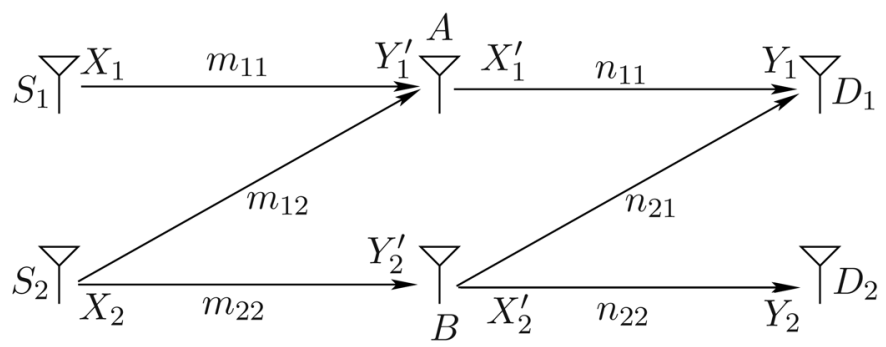

(b)

Fig. 4. $Z Z$ network. (a) The Gaussian $Z Z$ network. (b) The deterministic $Z Z$ network.

$$
\begin{aligned}
R_{1}+R_{2} & \leq m_{22}+\max \left(n_{11}, n_{21}\right), \\
R_{1}+R_{2} & \leq \max \left(m_{11}, m_{12}\right)+n_{22}, \\
R_{1} & \leq n_{11} \\
R_{2} & \leq \max \left(n_{21}, n_{22}\right), \\
R_{2} & \leq m_{22}+n_{21}, \\
R_{1}+R_{2} & \leq \max \left(n_{21}, n_{22}\right)+\left(n_{11}-n_{21}\right)^{+} .
\end{aligned}
$$

Here and elsewhere $(x)^{+}$denotes the positive part of $x$, which is formally defined by

$$
(x)^{+}= \begin{cases}x, & \text { if } x \geq 0 \\ 0, & \text { otherwise }\end{cases}
$$

Theorem 2 (Approximate Capacity Region of Gaussian ZS Network): Let $\mathcal{R}^{\mathrm{GZS}}$ be the set of all rate pairs $\left(R_{1}, R_{2}\right)$ which satisfy (GZS-1)-(GZS-10) given below. Then $\mathcal{R}^{\mathrm{GZS}}$ is an outer bound for the capacity region of the Gaussian ZS network. Moreover, for any $\left(R_{1}, R_{2}\right) \in \mathcal{R}^{\mathrm{GZS}}$, there exists a transmission scheme with rates $\left(R_{1}^{\prime}, R_{2}^{\prime}\right)=\left(\left(R_{1}-\delta_{1}\right)^{+},\left(R_{2}-\delta_{2}\right)^{+}\right)$, where $\delta_{1}=1$ and $\delta_{2}=1.5$ are universal constants, independent of the channel gain, and required rates.

$$
\begin{aligned}
R_{1} & \leq \frac{1}{2} \log \left(1+g_{11}\right) \\
R_{2} & \leq \frac{1}{2} \log \left(1+g_{12}+g_{22}\right) \\
R_{1}+R_{2} & \leq \frac{1}{2} \log \left(1+g_{11}+g_{12}\right)+\frac{1}{2} \log \left(1+\frac{g_{22}}{g_{12}}\right)
\end{aligned}
$$

$$
\begin{aligned}
R_{2} & \leq \frac{1}{2} \log \left(1+g_{12}\right)+\frac{1}{2} \log \left(1+h_{22}\right) \\
R_{1}+R_{2} & \leq \frac{1}{2} \log \left(1+g_{22}\right)+\frac{1}{2} \log \left(1+h_{11}+h_{21}\right) \\
R_{1}+R_{2} & \leq \frac{1}{2} \log \left(1+g_{11}+g_{12}\right)+\frac{1}{2} \log \left(1+h_{22}\right) \\
R_{1} & \leq \frac{1}{2} \log \left(1+h_{11}\right) \\
R_{2} & \leq \frac{1}{2} \log \left(1+h_{21}+h_{22}+2 \sqrt{h_{21} h_{22}}\right) \\
R_{2} & \leq \frac{1}{2} \log \left(1+g_{22}\right)+\frac{1}{2} \log \left(1+h_{21}\right) \\
R_{1}+R_{2} & \leq \frac{1}{2} \log \left(1+h_{21}+h_{22}+2 \sqrt{h_{21} h_{22}}\right) \\
& +\frac{1}{2} \log \left(1+\frac{h_{11}}{h_{21}}\right) .
\end{aligned}
$$

The outer bound for the results above are fairly standard arguments based on reducing a multiletter mutual information into single-letter forms by appropriately using decodability requirements at the different destinations. The details of these are given in Section VI-A and Appendix B.1, respectively.

The coding strategy achieving these regions is based on two ideas. One is that of a network decomposition illustrated in Example 1 in Section IV, for the deterministic network. The insight from the network decomposition leads to the idea of strategic rate-splitting and power allocation in the Gaussian channel. For the Gaussian coding scheme, we need to strategically partition the messages and allocate powers in order for the relays to partially decode appropriate messages and setup cooperation. The details of this strategy are outlined in Section V.

\section{B. ZZ Network}

The deterministic ZZ network illustrated in Fig. 4(b) and the corresponding Gaussian ZZ network is given in Fig. 4(a). Although superficially the ZS and ZZ networks may look similar, the subtle difference in the network connectivity, makes the two problems completely different, both in terms of capacity characterization, as well as transmission schemes. It will be shown that a new interference management scheme, which we term as interference neutralization, is needed to (approximately) achieve the capacity of this network. The most intuitive description for interference neutralization is to cancel interference over air without processing at the destinations. This scheme can be used whenever there are more than one path for interference to get received at a destination. We will explain it in more detail in Sections $\mathrm{V}$ and VII.

Theorems 3 and 4 give the exact and approximate (within 2 bits) characterizations for the capacity region of the deterministic and the Gaussian ZZ networks, respectively. Another new ingredient used here is a genie-aided outer bound that gives the output of the first (noisy) cross link of the first (or correspondingly second) layer to the first destination (or correspondingly to the relay). This genie-aided bound allows us to develop outer bounds that are apparantly tighter than the information-theoretic cut-set bounds by utilizing the decoding structure needed.

Theorem 3 (Capacity Region of Deterministic ZZ Network): The capacity region of the deterministic ZZ network is given by 
$\mathcal{R}^{\mathrm{DZZ}}$, where $\mathcal{R}^{\mathrm{DZZ}}$ is the set of all rate pairs $\left(R_{1}, R_{2}\right)$ which satisfy

$$
\begin{aligned}
R_{1} & \leq m_{11}, \\
R_{2} & \leq m_{22}, \\
R_{1} & \leq n_{11}, \\
R_{2} & \leq n_{22}, \\
R_{1}+r_{2} & \leq \max \left(m_{11}, m_{12}\right)+\left(m_{22}-m_{12}\right)^{+}+n_{12}
\end{aligned}
$$

(DZZ-5)

$$
R_{1}+R_{2} \leq \max \left(n_{11}, n_{12}\right)+\left(n_{22}-n_{12}\right)^{+}+m_{12}
$$

Theorem 4 (Approximate Capacity Region of Gaussian $\mathrm{ZZ}$ Network): Let $\mathcal{R}^{\mathrm{GZZ}}$ be the set of all rate pairs $\left(R_{1}, R_{2}\right)$ which satisfy (GZZ-1)-(GZZ-6) given below

$$
\begin{aligned}
R_{1} \leq & \frac{1}{2} \log \left(1+g_{11}\right) \\
R_{2} \leq & \frac{1}{2} \log \left(1+g_{22}\right) \\
R_{1} \leq & \frac{1}{2} \log \left(1+h_{11}\right) \\
R_{2} \leq & \frac{1}{2} \log \left(1+h_{22}\right) \\
R_{1}+R_{2} \leq & \frac{1}{2} \log \left(1+g_{11}+g_{12}\right)+\frac{1}{2} \log \left(1+\frac{g_{22}}{g_{12}}\right) \\
& +\frac{1}{2} \log \left(1+h_{12}\right), \\
R_{1}+R_{2} \leq & \frac{1}{2} \log \left(1+h_{11}+h_{12}\right)+\frac{1}{2} \log \left(1+\frac{h_{22}}{h_{12}}\right) \\
& +\frac{1}{2} \log \left(1+g_{12}\right) .
\end{aligned}
$$

Then, any admissible rate pair $\left(R_{1}, R_{2}\right)$ for the Gaussian $\mathrm{ZZ}$ network belongs to $\mathcal{R}^{\mathrm{GZZ}}$. Moreover, for any rate pair $\left(R_{1}, R_{2}\right) \in \mathcal{R}^{\mathrm{GZZ}}$, there exists an encoding scheme with rates $\left(R_{1}^{\prime}, R_{2}^{\prime}\right)=\left(\left(R_{1}-\frac{7}{4}\right)^{+},\left(R_{2}-\frac{7}{4}\right)^{+}\right)$.

\section{EXAMPLES ILLUSTRATING TRANSMISSION TECHNIQUES}

In this section, we illustrate through examples some of the main interference management techniques we will use to (approximately) achieve the capacity of our relay-interference networks. For simplicity in demonstrating the ideas, we focus on deterministic networks throughout the examples. However, similar techniques will be used later for Gaussian networks as well.

We also present a simple Gaussian example at the end of this section, to illustrate the message splitting idea used in many places throughout this work.

Example 1 (Network Decomposition for the ZS Network): A deterministic ZS network can be always decomposed into two sub-node-disjoint networks, where the first partition consists of a set of sub-nodes of $S_{1}, A$ and $D_{1}$, and looks like a line network. The second partition is, however, a diamond network, with a broadcast channel from $S_{2}$ to $A$ and $B$ in the first layer,

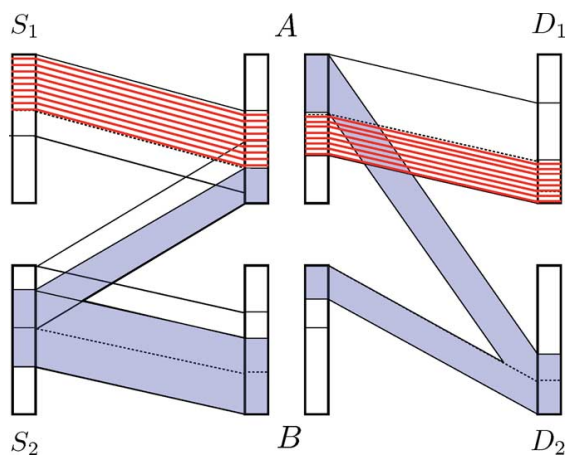

Fig. 5. Network partitioning for a deterministic ZS network.

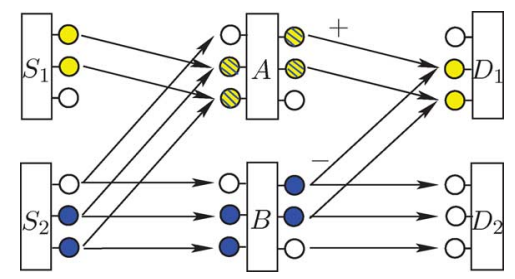

Fig. 6. Interference Neutralization; $\left(R_{1}, R_{2}\right)=(2,2)$ is achievable.

and a multiple access channel from $A$ and $B$ to $D_{2}$ in the second layer. This diamond network can be used to send information from $S_{2}$ to $D_{2}$. Since these two networks are sub-node disjoint, there would be no interfering signal, and each of them can be analyzed separately. This is more illustrated in Fig. 5.

In a Gaussian ZS network the network decomposition can be done using message splitting, superposition coding and proper power allocation. We will use this technique to achieve an approximate capacity for the Gaussian ZS network.

Example 2 (Interference Neutralization): This technique can be used in networks which contain more than one disjoint path from $S_{i}$ to $D_{j}$ for $i \neq j$, where $D_{j}$ is not interested in decoding the message sent by the source node $S_{i}$, and therefore, it receives the interference through more than one link. The proposed technique is to tune these interfering signals such that they neutralize each other at the destination node. In words, the interfering signal should be received at the same power level and with different signs such that the effective interference, obtained by adding them, occupies a smaller sub-space in the signal space of the receiver. To the best of our knowledge, this technique is new and has not been appeared in the literature before [17].

Fig. 6 shows a network in which interference neutralization is essential to achieve the desired rate pair $\left(R_{1}, R_{2}\right)=(2,3)$. Here $D_{1}$ has only two sub-nodes who can receive information from $A$. These two sub-nodes are also connected to $B$, and receive interference from $B$. However, notice that there are two disjoint paths $\left(S_{2}, A, D_{1}\right)$ and $\left(S_{2}, B, D_{1}\right)$, which connect $S_{2}$ to $D_{1}$. As it is shown in Fig. 6, using a proper mapping (permutation) at the relay nodes, one can make the interference neutralized at the destination node $D_{1}$, and provide two non-interfered links from $S_{1}$ to $D_{2}$. Note that this permutation does not effect the admissible rate of the other unicast from $S_{2}$ to $D_{2}$, and the cost we pay, is to permute the received bits at $D_{2}$. A more general illustration of this phenomenon is given in Fig. 7. 


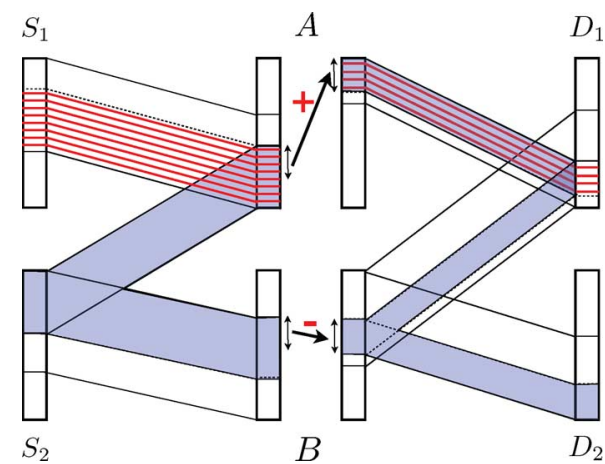

Fig. 7. Interference neutralization.

Example 3 (Use of Lattice Codes to Implement Interference Neutralization Over Gaussian ZZ Network): The idea of interference neutralization illustrated in Example 2 can be also used in Gaussian networks. In this case a group structured code, such as lattice code, is required to play the role of composition and decomposition of the signal and interference in two layers of the network. Consider the Gaussian ZZ network in Fig. 4(a). We can use message splitting and interference neutralization to improve the achievable rate pairs of this network.

Let the second source split its message into two parts as $W_{2}=$ $\left(W_{2}^{(N)}, W_{2}^{(P)}\right)$, namely, the functional (neutralization) and private parts, of rates $R_{2, N}=R_{1}$ and $R_{2, P}=R_{2}-R_{1}$. Both transmitters use a common lattice code to encode $W_{1}$ and $W_{2}^{(N)}$, and map them into $\mathbf{x}_{1}^{(N)}$ and $\mathbf{x}_{2}^{(N)}$, respectively. The other message $W_{2}^{(P)}$ can be encoded to $\mathbf{x}_{2}^{(P)}$ using a random Gaussian code. ${ }^{2}$ We assume that both the lattice code and the random Gaussian code have average power equal to 1 . Then, the transmitting signals would be a linear combination of the codewords with a proper power allocation, i.e.,

$$
\mathbf{x}_{1}=\sqrt{\alpha_{N}} \mathbf{x}_{1}^{(N)}, \quad \mathbf{x}_{2}=\sqrt{\beta_{N}} \mathbf{x}_{2}^{(N)}+\sqrt{\beta_{P}} \mathbf{x}_{2}^{(P)}
$$

where the power allocation coefficients satisfy $\alpha_{N} \leq 1$ and $\beta_{N}+\beta_{P} \leq 1$. The transmitters choose the power allocated to $\mathbf{x}_{1}^{(N)}$ to $\mathbf{x}_{2}^{(N)}$ in a way that they get received at $A$ with the same power. In this way, their summation would be again a lattice code and can be decoded at $A$ by treating $\mathbf{x}_{2}^{(P)}$ as noise. A similar strategy will be used for signaling at the relay for transmission in the second layer of the network. The only difference is that instead of sending $\mathbf{x}_{2}^{(N)}$, the relay node $B$ sends $-\mathbf{x}_{2}^{(N)}$. Then, the lattice point observed at $D_{1}$ would be exactly $\mathbf{x}_{1}^{(N)}$ and it can find $W_{1}$. The other decoder can simply first reverse $-\mathbf{x}_{2}^{(N)}$ to $\mathbf{x}_{2}^{(N)}$, and then decode it. This idea is illustrated in Fig. 8.

Example 4 (Gaussian Z Network): Consider the Gaussian Z network shown in Fig. 9, with channel gains $g_{11} \geq 1, g_{12} \geq 1$, and $g_{22} \geq 1$.

\footnotetext{
${ }^{2} \mathrm{~A}$ random code of rate $R$ and block length $\ell$ from distribution $\mathcal{P}(x)$ is a collection of $2^{\ell R}$ random sequences of length $\ell$, say $\left\{\mathbf{c}^{\ell}(i): i=1, \ldots, 2^{\ell R}\right\}$, each picked independently and randomly according to the distribution $\mathcal{P}\left(\mathbf{c}^{\ell}(i)\right)=\prod_{j=1}^{\ell} \mathcal{P}\left(\mathbf{c}_{j}(i)\right)$. In particular, a random Gaussian code is a random code whose codewords are element-wise picked independently from the Gaussian distribution with zero mean and certain variance.
}

The source nodes $F_{i}$ wishes to encode and send message $W_{i}$ to the destination node $G_{i}$, for $i=1,2$. Denoting the rate of message $W_{i}$ by $R_{i}$, an approximate capacity characterization for this network is given by

$$
\begin{aligned}
\mathcal{R}^{\mathrm{Z}}=\left\{\left(R_{1}, R_{2}\right)\right. & : R_{1} \leq \frac{1}{2} \log \left(1+g_{11}\right) \\
R_{2} & \leq \frac{1}{2} \log \left(1+g_{22}\right) \\
R_{1}+R_{2} & \left.\leq \frac{1}{2} \log \left(1+g_{11}+g_{12}\right)+\frac{1}{2} \log \left(1+\frac{g_{22}}{g_{12}}\right)\right\} .
\end{aligned}
$$

It is easy to show that any achievable rate pair belongs to $\mathcal{R}^{\mathrm{Z}}$, and hence, $\mathcal{R}^{\mathrm{Z}}$ establishes an outer bound for the capacity region. Moreover, one can show that the rate pair $\left(\left(R_{1}-\frac{1}{2}\right)^{+},\left(R_{2}-\frac{1}{2}\right)^{+}\right)$is achievable provided that $\left(R_{1}, R_{2}\right) \in \mathcal{R}^{\mathrm{Z}}$. The encoding strategy to achieve such rate pair involves message splitting and proper power allocation. We will discuss this in more details in Appendix C.

\section{GAUSSIAN Coding Strategies}

This section is devoted to providing the basic ideas of the coding schemes used in the Gaussian ZS and ZZ networks. We also develop an outline of how to analyze these coding strategies.

\section{A. Gaussian ZS Network: Achievability}

The coding strategy for the Gaussian ZS network is essentially a partial-decode-and-forward strategy, along with a strategic rate-splitting of the messages. Let the messages to be sent from $S_{1}, S_{2}$ be denoted by $W_{1}, W_{2}$, respectively [see Fig. 3(a)]. We will break the ZS network into two cascaded interference channels, where we require particular messages to be decoded at the relays and forwarded to the destinations. The first stage is a $Z$ interference channel, with messages $U_{1}^{(1)} \triangleq W_{1}$ and message $W_{2}$ which is split into three parts: $\left(U_{2}^{(1)}, U_{2}^{(2)}, U_{2}^{(3)}\right)$. The intention of this strategic split is to allow the node $G_{1}$ (which is relay $A$ in the original ZS network) to decode $\left(U_{1}^{(1)}, U_{2}^{(1)}, U_{2}^{(2)}\right)$ and node $G_{2}$ (which is relay $B$ in the original ZS network), to decode $\left(U_{2}^{(1)}, U_{2}^{(3)}\right)$. This is illustrated in Fig. 10. Here, $U_{2}^{(1)}$ plays the role of a common message which can be decoded at both receivers, whereas $U_{2}^{(2)}$ and $U_{2}^{(3)}$ are the private messages for $G_{1}$ and $G_{2}$, respectively.

The next stage of the ZS network is a $\mathrm{S}$ interference channel depicted in Fig. 11. Here we take the messages delivered and decoded by the $Z$ interference channel of the first stage and further process them to ensure delivery of the desired messages to the destination. In particular, we further split the decoded messages from the first stage into several parts and require delivery of messages as shown in Fig. 11. This splitting and delivery of appropriate pieces, finally ensures that $W_{1}$ and $W_{2}$ are decodable at the destinations. This is the encoding strategy in the ZS network. In the following lemmas, we give the rates at which messages at each stage can be delivered. Putting together Lemmas 1 and 2 , we get the desired result given in Theorem 2 . The proofs of 


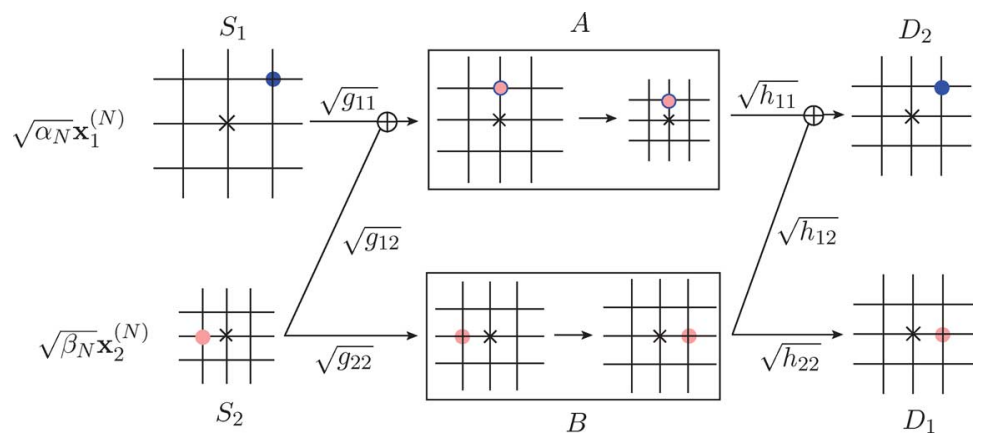

Fig. 8. Using lattice codes for interference neutralization over a Gaussian ZZ network. The origin is specified by a cross " $\times$ ". Power allocated to the messages at the transmitters are chosen such that the two lattice points corresponding to $\mathbf{x}_{1}^{(N)}$ and $\mathbf{x}_{2}^{(N)}$ get received at $B$ at the same power level, and their summation becomes a point on the scaled lattice. The same strategy is used by the relays. The relay node $B$ also reverses its transmitting lattice point in order to neutralize the interference caused in the first layer of the network.

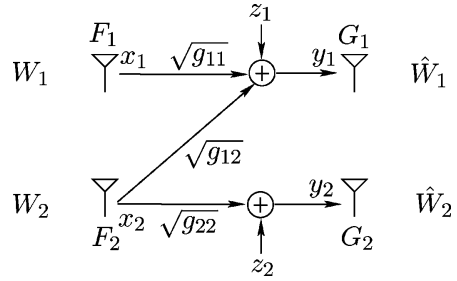

Fig. 9. Gaussian Z network.

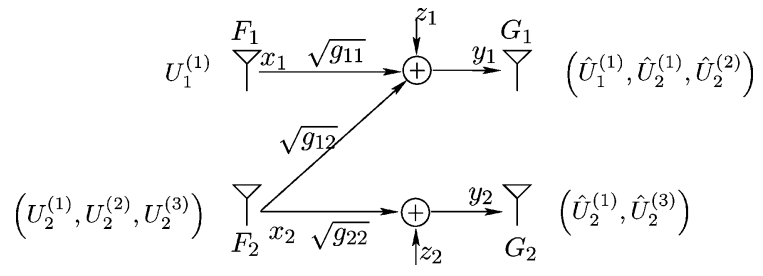

Fig. 10. Z interference channel with particular message requirements, captures the proposed coding scheme for the first layer of the Gaussian ZS network.

these lemmas follow fairly standard arguments, and are given in Appendix C.

A formal statement of the argument above is given below.

Lemma 1: Consider the Gaussian Z interference network with channel gains $\left(g_{11}, g_{12}, g_{22}\right)$, and decoding requirements as shown in Fig. 10. Denoting the rate of the sub-message $U_{i}^{(j)}$ by $\Upsilon_{i, j}$, any rate tuple $\left(\Upsilon_{1,1}, \Upsilon_{2,1}, \Upsilon_{2,2}, \Upsilon_{2,3}\right)$ which satisfies

$$
\begin{aligned}
\Upsilon_{1,1} & \leq\left(\frac{1}{2} \log \left(1+g_{11}\right)-\frac{1}{2}\right)^{+} \\
\Upsilon_{2,2} & \leq\left(\frac{1}{2} \log \left(1+\frac{g_{12}}{g_{22}}\right)-\frac{1}{2}\right)^{+} \\
\Upsilon_{2,1}+\Upsilon_{2,2} & \leq\left(\frac{1}{2} \log \left(1+g_{12}\right)-\frac{1}{2}\right)^{+} \\
\Upsilon_{1,1}+\Upsilon_{2,1}+\Upsilon_{2,2} & \leq\left(\frac{1}{2} \log \left(1+g_{11}+g_{12}\right)-\frac{1}{2}\right)^{+} \\
\Upsilon_{2,3} & \leq\left(\frac{1}{2} \log \left(1+\frac{g_{22}}{g_{12}}\right)-\frac{1}{2}\right)^{+} \\
\Upsilon_{2,1}+\Upsilon_{2,3} & \leq\left(\frac{1}{2} \log \left(1+g_{22}\right)-\frac{1}{2}\right)^{+}
\end{aligned}
$$

is achievable.
The next lemma gives an achievable rate region for the second layer of the ZS network, which is a S interference network depicted in Fig. 11.

Lemma 2: Consider the Gaussian S interference network with channel gains $\left(h_{11}, h_{21}, h_{22}\right)$, and decoding requirements as shown in Fig. 11, where $\Theta_{i, j}$ denotes the rate of message $V_{i}^{(j)}$. Any rate tuple $\left(\Theta_{1,1}, \Theta_{1,2}, \Theta_{2,1}, \Theta_{2,2}, \Theta_{2,3}, \Theta_{2,4}, \Theta_{2,5}\right)$ which satisfies

$$
\begin{aligned}
\Theta_{1,1}+\Theta_{1,2}+\Theta_{2,1} & +\Theta_{2,3} \\
\leq & \left(\frac{1}{2} \log \left(1+h_{11}\right)-\frac{1}{2}\right)^{+} \\
\Theta_{1,2} & \leq\left(\frac{1}{2} \log \left(1+\frac{h_{11}}{h_{12}}\right)-\frac{1}{2}\right)^{+} \\
\Theta_{2,4} & \leq\left(\frac{1}{2} \log \left(1+\frac{h_{21}}{h_{11}}\right)-\frac{1}{2}\right)^{+} \\
\Theta_{1,1}+\Theta_{2,3}+\Theta_{2,4} & \leq\left(\frac{1}{2} \log \left(1+h_{21}\right)-\frac{1}{2}\right)^{+} \\
\Theta_{2,5} & \leq\left(\frac{1}{2} \log \left(1+h_{22}\right)-\frac{1}{2}\right)^{+} \\
\Theta_{1,1}+\Theta_{2,1}+\Theta_{2,2} & +\Theta_{2,3}+\Theta_{2,4}+\Theta_{2,5} \\
\leq & \left(\frac{1}{2} \log \left(1+h_{21}+h_{22}\right)-\frac{1}{2}\right)^{+}
\end{aligned}
$$

is achievable.

\section{B. Gaussian ZZ Network: Achievability}

The encoding scheme needed for the ZZ network is slightly more sophisticated than the ZS network. An additional component to strategic message splitting is that of interference neutralization. This was illustrated in Examples 2 and 3 in Section IV. This along with message splitting inspired by the network decomposition illustrated in example 1 of Section IV, form the basis of the encoding scheme for the $\mathrm{ZZ}$ network.

More formally, the interference that has to be neutralized, will be combined with the main message in the first layer according to some partial-invertible function. In the second layer the inverse of the function is applied on this combination and the other interference received through the cross link. The remaining parts of the interference has to be either decoded or treated as noise. The neutralization is implemented using lattice 


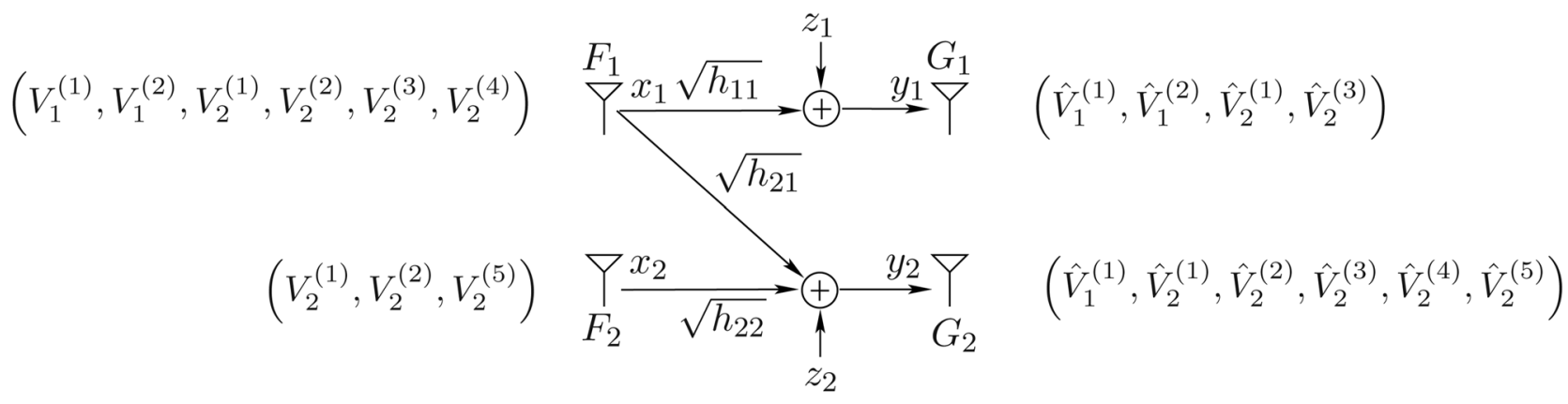

Fig. 11. S interference channel with particular message requirements, depicting the proposed coding strategy for the second layer of the Gaussian ZS network.

codes and the rate-splitting along with appropriate power allocation is also used.

We formally define a partial-invertible function and a Z-neutralization network in the following. The Gaussian $\mathrm{ZZ}$ network is essentially a cascade of two Z-neutralization networks. An achievable rate region for the Z-neutralization network is given in Lemma 3 . This rate region will be later used to obtain an achievable rate region for the Gaussian ZZ network. We will analyze the performance of the Gaussian encoding/decoding schemes in Appendix B.2.

Definition 1: Let $\mathcal{U}$ and $\mathcal{V}$ be two finite sets. A function $\phi(\cdot, \cdot)$ defined on $\mathcal{U} \times \mathcal{V}$ is called partial-invertible, if and only if having $\phi(u, v)$ and $u$, one can always reconstruct $v$ for any $u \in \mathcal{U}$ and $v \in \mathcal{V}$. Similarly, $u$ can be obtained from $\phi(u, v)$ and $v$.

An intuitive way of thinking about a partial-invertible $\phi(u, v)$ is the following. An arbitrary function defined on a finite sets $\mathcal{U}$ and $\mathcal{V}$ creates a table with rows corresponding to the elements of $\mathcal{U}$ and columns corresponding to the elements of $\mathcal{V}$, and each cell of the table consists the value assigned to its row and column by the function. A function will be partial-invertible, if and only if no two cells in the same column or row of its table be identical.

Note that summation over real numbers, and multiplication over nonzero numbers are two examples of partial-invertible functions. However, it is clear multiplication over real numbers is not partial-invertible, since $w=\phi(1,0)=\phi(2,0)$, and, therefore, having $w$ and $v=0, u$ can be anything.

Definition 2: Consider the Z network shown in Fig. 12, which consists of a Gaussian broadcast channel from $F_{2}$ to the receivers and a Gaussian multiple access channel from $F_{1}$ and $F_{2}$ to $G_{1}$. A Z-neutralization network is a Z network, wherein the first source node has two messages $\left(U_{1}^{(0)}, U_{1}^{(1)}\right)$ of rates $\Upsilon_{0}$ and $\Upsilon_{1}$, respectively. Similarly the second source observes two independent messages $\left(U_{2}^{(0)}, U_{2}^{(1)}\right)$ of rates $\Upsilon_{0}$ and $\Upsilon_{2}$.

The second receiver is interested in decoding $U_{2}^{(0)}$ and $U_{2}^{(1)}$, while the first destination wishes to decode $\phi\left(U_{1}^{(0)}, U_{2}^{(0)}\right)$ and $U_{1}^{(1)}$, where $\phi(\cdot, \cdot)$ can be any arbitrary partial-invertible function. A rate tuple $\left(\Upsilon_{0}, \Upsilon_{1}, \Upsilon_{2}\right)$ is called achievable if the receivers can decode their messages with arbitrary small error probability.

Lemma 3: Consider the Z-neutralization network defined in Definition 2 with channel gains $\left(g_{11}, g_{12}, g_{22}\right)$ (see Fig. 12). Let

$$
\lambda \triangleq \min \left\{g_{11}, g_{12}, g_{22}\right\}
$$

and

$$
\mu \triangleq \max \left\{g_{11}, g_{12}, g_{22}, \frac{g_{11} g_{22}}{g_{12}}\right\} \text {. }
$$

Any rate tuple $\left(\Upsilon_{0}, \Upsilon_{1}, \Upsilon_{2}\right)$ satisfying

$$
\begin{aligned}
\Upsilon_{0} & \leq\left(\frac{1}{2} \log (\lambda)-\frac{1}{2}\right)^{+} \\
\Upsilon_{0}+\Upsilon_{1} & \leq\left(\frac{1}{2} \log \left(g_{11}\right)-1\right)^{+} \\
\Upsilon_{0}+\Upsilon_{2} & \leq\left(\frac{1}{2} \log \left(g_{22}\right)-1\right)^{+} \\
\Upsilon_{0}+\Upsilon_{1}+\Upsilon_{2} & \leq\left(\frac{1}{2} \log (\mu)-\frac{3}{2}\right)^{+}
\end{aligned}
$$

is achievable.

As mentioned before, we strategically split the messages and require functional reconstructions for some of them at the relay nodes to facilitate neutralization at the destinations. More precisely, in the first layer of the network, each source node splits its message into two parts, namely, "functional" and "private" parts, $W_{1}=\left(U_{1}^{(0)}, U_{1}^{(1)}\right)$ and $W_{2}=\left(U_{2}^{(0)}, U_{2}^{(1)}\right)$. The functional parts $U_{1}^{(0)}, U_{2}^{(0)}$ both have the same rates $\Upsilon_{0}$. Both transmitters use a common lattice code to encode their functional sub-messages. Now the first layer encodes the message such that the first receiver (which is relay $A$ in the original $\mathrm{ZZ}$ network) can decode $U_{1}^{(1)}$ and $\phi\left(U_{1}^{(0)}, U_{2}^{(0)}\right)$, and the second one (relay $B$ in the original ZZ network) can decode $U_{2}^{(0)}$ and $U_{2}^{(1)}$. Lemma 3 gives the rates at which these can be sent reliably. The second stage operates in a manner similar to the first stage, by splitting the messages into functional and private parts. The first sender (relay $A$ in the original network) uses $U_{1}^{(1)}$ and $\phi\left(U_{1}^{(0)}, U_{2}^{(0)}\right)$ as the private and functional parts and the other one (relay $B$ ) uses $U_{2}^{(1)}$ and $U_{2}^{(0)}$ as the private and functional parts.

The functional parts are sent appropriately, using a common lattice code in both stages. Let $\mathbf{x}_{1}^{(N)}$ and $\mathbf{x}_{2}^{(N)}$ be the lattice codewords, corresponding to $U_{1}^{(0)}$ and $U_{2}^{(0)}$, respectively. The power allocation in the first layer is done so that two lattice points get received at $A$ at the same power (see Fig. 8). The group structure of the lattice code implies that the summation of two received lattice point, $\tilde{\mathbf{x}}^{(N)}=\mathbf{x}_{1}^{(N)}+\mathbf{x}_{2}^{(N)}$ is still a valid codeword, and can be decoded by $A$. The function $\phi(\cdot, \cdot)$ is in fact the decoded message from $\tilde{\mathbf{x}}^{(N)}$. In the second stage, relay node $B$, sends the inverse of the the received lattice point, that is $\mathbf{x}_{2}^{\prime(N)}=-\mathbf{x}_{2}^{(N)}$, while $A$ forwards the sum lattice point, $\mathbf{x}_{1}^{\prime(N)}=\tilde{\mathbf{x}}^{(N)}$. Again 


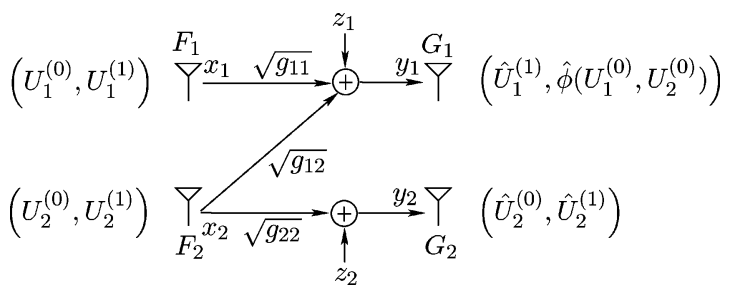

Fig. 12. Gaussian $Z$ channel.

these lattice points are scaled properly so that they get received at $D_{1}$ at the same power. Thus, their summation would be a lattice point and equals $\mathbf{x}_{1}^{\prime(N)}+\mathbf{x}_{2}^{\prime(N)}=\left(\mathbf{x}_{1}^{(N)}+\mathbf{x}_{2}^{(N)}\right)-\mathbf{x}_{2}^{(N)}=$ $\mathbf{x}_{1}^{(N)}$, which will be decoded to $U_{1}^{(0)}$. The other destination $D_{2}$, receives $-\mathbf{x}_{2}^{(N)}$, finds its inverse $\mathbf{x}_{2}^{(N)}$, and finally decodes it to $U_{2}^{(0)}$. This idea is illustrated in Example 3, and the precise details of this argument are given in Appendix B.2.

\section{Deterministic ZS NeTWORK}

In this section, we prove Theorem 1 . We present this proof in two parts. First we present the converse proof, which shows any achievable rate pair belongs to $\mathcal{R}^{\mathrm{DZS}}$. Then for any rate pair in this region, we propose an encoding scheme which is able to transmit messages up to the desired rates.

\section{A. Outer Bound}

In this section we show that any achievable rate pair $\left(R_{1}, R_{2}\right)$ for the deterministic ZS network belongs to $\mathcal{R}^{\mathrm{DZS}}$. Assume there exists a coding scheme with block length $\ell$ which can be used to communicate at rates $R_{1}$ and $R_{2}$ over the network. We use bold face matrices to denote $\ell$ copy of them, as the transfer matrix applied over a codeword of length $\ell$. Such matrices can be written as the Kronecker product of identity matrix of size $\ell$ and the single letter transfer matrix of the channel, e.g., $\mathbf{M}_{11}=I_{\ell} \otimes$ $M_{11}$. Here, the Kronecker product of matrices $A_{m \times n}=\left(a_{i, j}\right)$ and $B_{p \times q}=\left(b_{i, j}\right)$ is defined as $C_{m p \times n q}=A \otimes B$, where

$$
C_{\left[\left(m^{\prime}-1\right) p+p^{\prime}\right],\left[\left(n^{\prime}-1\right) q+q^{\prime}\right]}=a_{m^{\prime}, n^{\prime}} \cdot b_{p^{\prime}, q^{\prime}}
$$

for $m^{\prime}=1, \ldots, m, n^{\prime}=1, \ldots, n, p^{\prime}=1, \ldots, p$, and $q^{\prime}=$ $1, \ldots, q$.

The following simple lemma which relates the entropy of a random projected vector to the rank of the projection matrix will be frequently used in the following proof. We will prove this lemma in Appendix C.

Lemma 4: For any matrix $G_{1}$, the entropy of $G_{1} X$ can be upper bounded by

$$
H\left(G_{1} X\right) \leq \operatorname{rank}\left(G_{1}\right) .
$$

Moreover, for matrices $G_{1}$ and $G_{2}$, we have

$$
H\left(G_{1} X \mid G_{2} X\right) \leq \operatorname{rank}\left[\begin{array}{l}
G_{1} \\
G_{2}
\end{array}\right]-\operatorname{rank}\left(G_{2}\right) .
$$

All of the bounds in Theorem 1 except (DZS-3) and (DZS-10) can be obtained straight-forwardly using the generalized cut-set bound in [18], which shows that in a linear finite-field network, the maximum reliable rate can be transmitted through a cut is upper bounded by the rank of the transition matrix of the cut. Here, we only present the proof of (DZS-5) to illustrate this idea.
Then we prove the two remaining bounds, which are tighter than the cut-set bound.

(DZS-5) $R_{1}+R_{2} \leq m_{22}+\max \left(n_{11}, n_{21}\right)$ : Recall the notation used in Fig. 3(b) for transmitting and receiving signals. This bound corresponds to the cut $\Omega_{s}=\left\{S_{1}, S_{2}, A\right\}$ and $\Omega_{d}=\left\{B, D_{1}, D_{2}\right\}$. The transition matrix from the input of the cut $X_{\Omega_{s}}=\left(X_{2}, X_{1}^{\prime}\right)$ to its output $Y_{\Omega_{d}}=\left(Y_{2}^{\prime}, Y_{1}, Y_{2}\right)$ can be written as

$$
\left[\begin{array}{c}
Y_{2}^{\prime \ell} \\
Y_{1}^{\ell} \\
Y_{2}^{\ell}
\end{array}\right]=\underbrace{\left[\begin{array}{cc}
\mathbf{M}_{22} & \mathbf{0} \\
\mathbf{0} & \mathbf{N}_{11} \\
\mathbf{0} & \mathbf{N}_{21}
\end{array}\right]}_{\mathbf{G}_{\Omega_{s}, \Omega_{d}}}\left[\begin{array}{c}
X_{2}^{\ell} \\
X_{1}^{\prime \ell}
\end{array}\right]+\left[\begin{array}{c}
\mathbf{0} \\
\mathbf{0} \\
\mathbf{N}_{22}
\end{array}\right] X_{2}^{\prime \ell} .
$$

Therefore, from [18], we have

$$
\begin{aligned}
\ell\left(R_{1}+R_{2}\right) & \leq \operatorname{rank}\left(\mathbf{G}_{\Omega_{s}, \Omega_{d}}\right)=\operatorname{rank}\left(\mathbf{M}_{22}\right)+\operatorname{rank}\left[\begin{array}{c}
\mathbf{N}_{11} \\
\mathbf{N}_{21}
\end{array}\right] \\
& =\ell m_{22}+\ell \max \left(n_{11}, n_{21}\right) .
\end{aligned}
$$

As mentioned before, we skip the proof of those bounds which follow from the generalized cut-set bound. In the following we present the proof of the two remaining inequalities which are tighter that the cut-set bound.

(DZS-3) $R_{1}+R_{2} \leq \max \left(m_{11}, m_{12}\right)+\left(m_{22}-m_{12}\right)^{+}:$In order to prove this bound, we can start with

$$
\begin{aligned}
\ell\left(R_{1}+R_{2}\right) \leq & I\left(X_{1}^{\ell}, X_{2}^{\ell} ; Y_{1}^{\ell}, Y_{2}^{\ell}\right) \\
\leq & I\left(X_{1}^{\ell}, X_{2}^{\ell} ; Y_{1}^{\prime \ell}, Y_{2}^{\prime \ell}\right) \\
= & I\left(X_{1}^{\ell}, X_{2}^{\ell} ; Y_{1}^{\prime \ell}\right)+I\left(X_{1}^{\ell}, X_{2}^{\ell} ; Y_{2}^{\prime \ell} \mid Y_{1}^{\prime \ell}\right) \\
= & I\left(X_{1}^{\ell}, X_{2}^{\ell} ; Y_{1}^{\prime \ell}\right)+H\left(Y_{2}^{\prime \ell} \mid Y_{1}^{\prime \ell}\right) \\
& -H\left(Y_{2}^{\prime \ell} \mid X_{1}^{\ell}, X_{2}^{\ell}, Y_{1}^{\prime \ell}\right) \\
= & I\left(X_{1}^{\ell}, X_{2}^{\ell} ; Y_{1}^{\prime \ell}\right)+H\left(Y_{2}^{\prime \ell} \mid Y_{1}^{\prime \ell}\right)
\end{aligned}
$$

where in (31) we used the data

$$
\left(X_{1}^{\ell}, X_{2}^{\ell}\right) \leftrightarrow\left(Y_{1}^{\prime \ell}, Y_{2}^{\prime \ell}\right) \leftrightarrow\left(X_{1}^{\prime \ell}, X_{2}^{\prime \ell}\right) \leftrightarrow\left(Y_{1}^{\ell}, Y_{2}^{\ell}\right)
$$

and (32) holds since $Y_{2}^{\prime \ell}$ is a deterministic function of $X_{2}^{\ell}$ in the $Z$ part of the network. Now, using Lemma 4 it is clear that

$I\left(X_{1}^{\ell}, X_{2}^{\ell} ; Y_{1}^{\prime \ell}\right) \leq \operatorname{rank}\left[\mathbf{M}_{11} \quad \mathbf{M}_{21}\right]=\ell \max \left(m_{11}, m_{12}\right)$.

In order to bound the second term, we can write

$$
\begin{aligned}
H\left(Y_{2}^{\prime \ell} \mid Y_{1}^{\prime \ell}\right) & =H\left(Y_{2}^{\prime \ell} \mid Y_{1}^{\prime \ell}, X_{1}^{\prime \ell}, Y_{1}^{\ell}\right) \\
& \leq H\left(Y_{2}^{\prime \ell}, W_{1} \mid Y_{1}^{\prime \ell}, X_{1}^{\prime \ell}, Y_{1}^{\ell}\right) \\
& =H\left(Y_{2}^{\prime \ell} \mid Y_{1}^{\prime \ell}, X_{1}^{\prime \ell}, Y_{1}^{\ell}, W_{1}\right)+H\left(W_{1} \mid Y_{1}^{\prime \ell}, X_{1}^{\prime \ell}, Y_{1}^{\ell}\right) \\
& \leq H\left(Y_{2}^{\prime \ell} \mid Y_{1}^{\prime \ell}, X_{1}^{\prime \ell}, Y_{1}^{\ell}, W_{1}\right)+\ell \varepsilon_{\ell} \\
& =H\left(Y_{2}^{\prime \ell} \mid Y_{1}^{\prime \ell}, X_{1}^{\prime \ell}, Y_{1}^{\ell}, W_{1}, X_{1}^{\ell}\right)+\ell \varepsilon_{\ell} \\
& \leq H\left(Y_{2}^{\prime \ell} \mid Y_{1}^{\prime \ell}-\mathbf{M}_{11} X_{1}^{\ell}\right)+\ell \varepsilon_{\ell} \\
& =H\left(\mathbf{M}_{22} X_{2}^{\ell} \mid \mathbf{M}_{12} X_{2}^{\ell}\right)+\ell \varepsilon_{\ell} \\
& \leq \ell \operatorname{rank}\left[\begin{array}{l}
M_{12} \\
M_{22}
\end{array}\right]-\ell \operatorname{rank}\left(M_{12}\right)+\ell \varepsilon_{\ell} \\
& =\ell \max \left(m_{12}, m_{22}\right)-\ell m_{12}+\ell \varepsilon_{\ell} \\
& =\ell\left(m_{22}-m_{12}\right)^{+}+\ell \varepsilon_{\ell}
\end{aligned}
$$

where (35) holds since $X_{1}^{\prime \ell}$ is completely determined by $Y_{1}^{\prime \ell}$, and $Y_{1}^{\ell}$ is also a deterministic function of $X_{1}^{\prime \ell}$ in the $S$ part of the network. We used Fano's inequality in (36), where $W_{1}$ should 
be decodable based on $Y_{1}^{\ell}$. Here, $\varepsilon_{\ell} \rightarrow 0$ as $\ell$ grows. Equality in (37) holds, since $X_{1}^{\ell}$ is determined by $W_{1}$, and finally, we used Lemma 4 in (38). Summing up (34) and (39), we get the desired bound.

Note that the cut-set bound for the cut $\Omega_{s}=\left\{S_{1}, S_{2}\right\}$ and $\Omega_{d}=\left\{A, B, D_{1}, D_{2}\right\}$ gives us

$$
\ell\left(R_{1}+R_{2}\right) \leq \operatorname{rank}\left[\begin{array}{cc}
\mathbf{M}_{11} & \mathbf{M}_{12} \\
\mathbf{0} & \mathbf{M}_{22}
\end{array}\right]=\ell \max \left(m_{11}+m_{22}, m_{12}\right)
$$

in which the RHS can be arbitrarily larger than the RHS of the presented bound. The reason for this difference is the following. It is inherently assumed in deriving the cut-set bound that the receivers can cooperate to decode the messages of rates $R_{1}$ and $R_{2}$, and no decodability requirement is posed for individual receivers. However, the setup of this problem imposes an extra constraint, that is $B$ alone should be able to decode $W_{2}$. Incorporating this decodability requirement shrinks the set of admissible rates, and gives us a tighter bound.

(DZS-10) $R_{1}+R_{2} \leq \max \left(n_{21}, n_{11}\right)+\left(n_{11}-n_{21}\right)^{+}$: The last inequality captures the maximum flow of information from the relays to the destinations, such that $D_{1}$ and $D_{2}$ be able to decode $W_{1}$ and $W_{2}$, respectively. We again start with

$$
\begin{aligned}
\ell\left(R_{1}+R_{2}\right) & \leq I\left(X_{1}^{\ell}, X_{2}^{\ell} ; Y_{1}^{\ell}, Y_{2}^{\ell}\right)=H\left(Y_{1}^{\ell}, Y_{2}^{\ell}\right) \\
& =H\left(Y_{2}^{\ell}\right)+H\left(Y_{1}^{\ell} \mid Y_{2}^{\ell}\right) .
\end{aligned}
$$

The first term can be easily bounded by

$$
H\left(Y_{2}^{\ell}\right) \leq \operatorname{rank}\left[\begin{array}{ll}
\mathbf{N}_{21} & \mathbf{N}_{22}
\end{array}\right]=\ell \max \left(n_{21}, n_{22}\right) .
$$

In order to bound the second term, we use the fact that $W_{2}$ can be decoded from $Y_{2}^{\ell}$. Therefore

$$
\begin{aligned}
H\left(Y_{1}^{\ell} \mid Y_{2}^{\ell}\right) & \leq H\left(Y_{1}^{\ell}, W_{2} \mid Y_{2}^{\ell}\right) \\
& =H\left(Y_{1}^{\ell} \mid Y_{2}^{\ell}, W_{2}\right)+H\left(W_{2} \mid Y_{2}^{\ell}\right) \\
& \leq H\left(Y_{1}^{\ell} \mid Y_{2}^{\ell}, W_{2}\right)+\ell \varepsilon_{\ell} \\
& =H\left(Y_{1}^{\ell} \mid Y_{2}^{\ell}, W_{2}, X_{2}^{\ell}, Y_{2}^{\prime \ell}, X_{2}^{\prime \ell}\right)+\ell \varepsilon_{\ell} \\
& \leq H\left(Y_{1}^{\ell} \mid Y_{2}^{\ell}-\mathbf{N}_{22} X_{2}^{\prime \ell}\right)+\ell \varepsilon_{\ell} \\
& =H\left(\mathbf{N}_{11} X_{1}^{\prime \ell} \mid \mathbf{N}_{21} X_{1}^{\prime \ell}\right)+\ell \varepsilon_{\ell} \\
& \leq \ell \operatorname{rank}\left[\begin{array}{l}
N_{11} \\
N_{21}
\end{array}\right]-\ell \operatorname{rank}\left(N_{21}\right)+\ell \varepsilon_{\ell} \\
& =\ell\left(n_{11}-n_{21}\right)^{+}+\ell \varepsilon_{\ell} .
\end{aligned}
$$

In (43) we used the Fano's inequality, as well as the fact that $X_{2}^{\ell}$, $Y_{2}^{\prime \ell}$, and $X_{2}^{\prime \ell}$ are known having $W_{2}$. We again used Lemma 4 in (44). Finally, the desired bound is obtained by replacing (42) and (45) in (41).

It is worth mentioning that this bound is tighter than the cut-set bound for the cut $\Omega_{s}=\left\{S_{1}, S_{2}, A, B\right\}$ and $\Omega_{d}=\left\{D_{1}, D_{2}\right\}$, which is

$$
R_{1}+R_{2} \leq \max \left(n_{11}+n_{22}, n_{12}\right)
$$

\section{B. Achievability Proof}

Network Decomposition: The achievability scheme presented here is based on decomposition of the deterministic ZS network into two sub-node-disjoint networks. In fact, such partitioning depends on the demanded rate pair $\left(R_{1}, R_{2}\right) \in \mathcal{R}^{\mathrm{DZS}}$. The resulting family of separations immediately suggests a simple coding scheme. We will show that this separation is optimal, and does not cause any loss in the admissible rate region of the network.

Before introducing the network decomposition, we define an equivalence class for the sub-nodes (levels) in a network.

Definition 3: In a Z (or S) deterministic network, two subnodes $a$ and $b$ are called related sub-nodes, and denoted by $a \sim b$ if any of the following conditions hold:

- $a=b$;

- $a$ is connected to $b$;

- $b$ is connected to $a$;

- there exists a sub-node $c$ such that $c$ broadcasts to both $a$ and $b$;

- there exists a sub-node $d$ where both $a$ and $b$ are connected to.

Note that this relation is reflective, symmetric, and transitive. Therefore, it forms equivalence classes for the sub-nodes.

We denote by $\mathcal{N}_{1}$ and $\mathcal{N}_{2}$ the partitions of the network. Assume we wish transmitting at rate $R_{1} \triangleq r \leq \min \left(m_{11}, n_{11}\right)$ from $S_{1}$ to $D_{1}$. The first part of the network $\mathcal{N}_{1}$, includes the top $\left(m_{11}-m_{12}\right)^{+}$levels as well as the lowest $\left(r-\left(m_{11}-m_{12}\right)^{+}\right)^{+}$ levels of $S_{1}$ who are connected to $A$. It also includes all the related sub-nodes of $S_{2}$, and the receiver levels of $A$ and $B$. Similarly, in the second layer of the network, $\mathcal{N}_{1}$ includes the lowest $\left(n_{11}-n_{21}\right)^{+}$levels as well as the top $\left(r-\left(n_{11}-n_{21}\right)^{+}\right)^{+}$nodes of the transmitter part of $A$. All related sub-nodes of the transmitter part of $B$, as well as $D_{1}$ and $D_{2}$ also belong to $\mathcal{N}_{1}$. The second part of the network $\mathcal{N}_{2}$, is formed by all the remaining nodes.

We will use $\mathcal{N}_{1}$ for transmitting data from $S_{1}$ to $D_{1}$. Similarly $\mathcal{N}_{2}$ is only used to communicate from $S_{2}$ to $D_{2}$. Therefore, we have two unicast networks, and each pair of transmitter-receiver can communicate up to the capacity of their own partition, which is the min-cut of the partition [6].

It is worth mentioning that any two "related" sub-nodes belong to the same partition. Therefore, these two networks are sub-node-disjoint, and do not cause interference for each other. This allows us to derive the capacity of each network separately, and argue that $\left(R_{1}, R_{2}\right)$ can be achieved simultaneously for the original network, if $R_{1}$ and $R_{2}$ are achievable for partitions $\mathcal{N}_{1}$ and $\mathcal{N}_{2}$.

Encoding Scheme: A transmission from $S_{1}$ and $S_{2}$ to $D_{1}$ and $D_{2}$ is performed as follows. $S_{1}$ transmits only on its subnodes which belong to $\mathcal{N}_{1}$, and keeps its other sub-nodes silent. Similarly, $S_{2}$ encodes its message on the sub-nodes included in $\mathcal{N}_{2}$, and sends zero on the other levels. Therefore, the effective communication over each partition is a simple unicast.

Fig. 13 shows the effective parts of the network. It is easy to see that the diamond network in Fig. 13(b) is also a linear shift deterministic networks. However, note that the lowest $(r-$ 


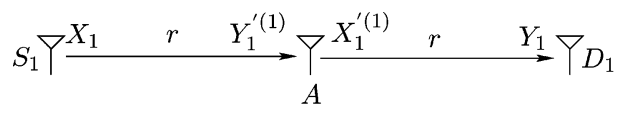

(a)

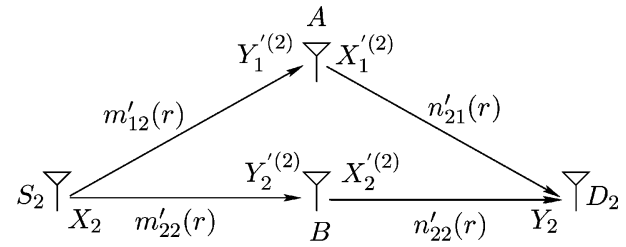

(b)

Fig. 13. Effective separated ZS network. (a) Effective channel for $\left(S_{1}, D_{1}\right)$. (b) Effective channel for $\left(S_{2}, D_{2}\right)$.

$\left.\left(m_{11}-m_{12}\right)^{+}\right)^{+}$levels of $A$ belong to $\mathcal{N}_{1}$. Therefore, the gain of the channel from $S_{2}$ to $A$ in $\mathcal{N}_{2}$ can be computed as

$$
\begin{aligned}
m_{12}^{\prime}(r) & =m_{12}-\left(r-\left(m_{11}-m_{12}\right)^{+}\right)^{+} \\
& =m_{12}-\max \left(r-\left(m_{11}-m_{12}\right)^{+}, 0\right) \\
& =\min \left(m_{12}+\left(m_{11}-m_{12}\right)^{+}-r, m_{12}\right) \\
& =\min \left(\max \left(m_{11}, m_{12}\right)-r, m_{12}\right) .
\end{aligned}
$$

Similarly, the other channel gains can be written as

$$
\begin{aligned}
m_{22}^{\prime}(r) & =\min \left(\max \left(m_{11}, m_{12}\right)+\left(m_{22}-m_{12}\right)^{+}-r, m_{22}\right) \\
n_{21}^{\prime}(r) & =\min \left(\max \left(n_{11}, n_{21}\right)-r, n_{21}\right) \\
n_{22}^{\prime}(r) & =\min \left(\max \left(n_{11}, n_{21}\right)+\left(n_{22}-n_{21}\right)^{+}-r, n_{22}\right) .
\end{aligned}
$$

Achievable Rate Region: The cut values of $\mathcal{N}_{1}$ can be easily computed as

$$
\begin{aligned}
\Omega=\left\{S_{1}\right\}: & \left(m_{11}-m_{12}\right)^{+}+\left(r-\left(m_{11}-m_{12}\right)^{+}\right)^{+} \\
& =\max \left\{\left(m_{11}-m_{12}\right)^{+}, r\right\} \geq r \\
\Omega=\left\{S_{1}, A\right\}: & \left(r-\left(n_{11}-n_{12}\right)^{+}\right)^{+}+\left(n_{11}-n_{12}\right)^{+} \\
= & \max \left\{\left(n_{11}-n_{12}\right)^{+}, r\right\} \geq r .
\end{aligned}
$$

Therefore, any rate in $\mathcal{R}_{1}^{\mathrm{DZS}}(r)=\left\{R_{1}: R_{1} \leq r\right\}$ can be conveyed from $S_{1}$ to $D_{1}$ through $\mathcal{N}_{1}$.

The capacity of $\mathcal{N}_{2}$ can be found using the generalized max-flow min-cut theorem [6]. Hence, the rate region of the second partition $\mathcal{N}_{2}$ would be

$$
\begin{aligned}
\mathcal{R}_{2}^{\mathrm{DZS}}(r)=\left\{R_{2}:\right. & R_{2} \leq \max \left(m_{12}^{\prime}(r), m_{22}^{\prime}(r)\right) \\
& R_{2} \leq m_{22}^{\prime}(r)+n_{21}^{\prime}(r) \\
& R_{2} \leq m_{12}^{\prime}(r)+n_{22}^{\prime}(r) \\
& \left.R_{2} \leq \max \left(n_{21}^{\prime}(r), n_{22}^{\prime}(r)\right)\right\} .
\end{aligned}
$$

Therefore, by using this decomposition, any rate pair in the set $\mathcal{R}_{1}^{\mathrm{DZS}}(r) \times \mathcal{R}_{2}^{\mathrm{DZS}}(r)=\left\{\left(R_{1}, R_{2}\right): R_{1} \in \mathcal{R}_{1}^{\mathrm{DZS}}(r), R_{2} \in\right.$ $\left.\mathcal{R}_{2}^{\mathrm{DZS}}(r)\right\}$ can be achieved. It remains to prove the following lemma.

Lemma 5: For any deterministic ZS network

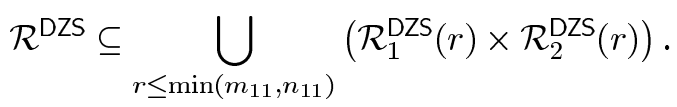

We will prove this lemma in Appendix C.

\section{DETERMINISTIC ZZ NETWORK}

In this section, we prove Theorem 3 . This is done in two parts, that provides the converse and achievability proofs.

\section{A. Outer Bound}

In the following we will show that any achievable rate pair $\left(R_{1}, R_{2}\right)$ satisfies constraints (DZZ-1)-(DZZ-6). The individual rate bounds can be directly obtained by the generalized cut-set bound introduced in [18], where the maximum flow of information through a cut in a linear deterministic network is upper bounded by the rank of the transition matrix from the sender part of the cut to its receiver part. Hence, we skip the proofs of (DZZ-1)-(DZZ-4).

The sum-rate bounds in (DZZ-5)-(DZZ-6) are, however, genie-aided bounds which are tighter that the cut-set bounds. In the following, we focus on these two bounds, and present their proofs in detail. Again we assume that there exists a coding scheme with block length $\ell$ which can be used to communicate at rates $R_{1}$ and $R_{2}$ over the network.

(DZZ-5) $R_{1}+R_{2} \leq \max \left(m_{11}, m_{12}\right)+\left(m_{22}-m_{12}\right)^{+}+$ $n_{12}$ : In order to prove this inequality we focus on the flow of information from the sources to the relays. The key idea here is to provide $A$ with the information sent by $B$ to $D_{1}$, as side information. In such condition, the information $A$ has received about $W_{1}$ is stronger than the information available at $D_{1}$, and, therefore, $A$ can decode $W_{1}$ since $D_{1}$ can do as well. Once $W_{1}$ is decoded at $A$, it can determine the transmitted codeword from $S_{1}$. By removing the interference from $S_{1}, A$ can also partially decode $W_{2}$.

More precisely, we can write

$$
\begin{aligned}
\ell\left(R_{1}+R_{2}\right) & \leq I\left(X_{1}^{\ell}, X_{2}^{\ell} ; Y_{1}^{\prime \ell}, Y_{2}^{\prime \ell}\right)=H\left(Y_{1}^{\prime \ell}, Y_{2}^{\prime \ell}\right) \\
& \leq H\left(Y_{1}^{\prime \ell}, Y_{2}^{\prime \ell}, \Gamma_{2}^{\ell}\right) \\
& =H\left(Y_{1}^{\prime \ell}, \Gamma_{2}^{\ell}\right)+H\left(Y_{2}^{\prime \ell} \mid Y_{1}^{\prime \ell}, \Gamma_{2}^{\ell}\right) \\
& \leq H\left(Y_{1}^{\prime \ell}\right)+H\left(\Gamma_{2}^{\ell}\right)+H\left(Y_{2}^{\prime \ell} \mid Y_{1}^{\prime \ell}, \Gamma_{2}^{\ell}\right)
\end{aligned}
$$

where $\Gamma_{2}^{\ell}=\mathbf{N}_{12} X_{2}^{\prime \ell}$ is the part of the signal received at $D_{2}$ from $B$ as in Fig. 4(b). The first two terms are easily bounded by $\ell \max \left(m_{11}, m_{12}\right)$ and $\ell n_{12}$, respectively. Deriving an upper bound for the last term is more involved.

Similar to $\Gamma_{2}^{\ell}$, we define $\Gamma_{1}^{\ell}=\mathbf{M}_{12} X_{2}^{\ell}=Y_{1}^{\prime \ell}-\mathbf{M}_{11} X_{1}^{\ell}$, where we have

$$
\begin{aligned}
H\left(\Gamma_{1}^{\ell} \mid Y_{1}^{\prime \ell}, \Gamma_{2}^{\ell}\right) & =H\left(Y_{1}^{\prime \ell}-\mathbf{M}_{11} X_{1}^{\ell} \mid Y_{1}^{\prime \ell}, \Gamma_{2}^{\ell}\right) \\
& =H\left(\mathbf{M}_{11} X_{1}^{\ell} \mid Y_{1}^{\prime \ell}, \Gamma_{2}^{\ell}\right) \\
& \leq H\left(X_{1}^{\ell} \mid Y_{1}^{\prime \ell}, \Gamma_{2}^{\ell}\right) \\
& \leq H\left(W_{1} \mid Y_{1}^{\prime \ell}, \Gamma_{2}^{\ell}\right) \\
& =H\left(W_{1} \mid Y_{1}^{\prime \ell}, X_{1}^{\prime \ell}, \Gamma_{2}^{\ell}\right) \\
& \leq H\left(W_{1} \mid \mathbf{N}_{11} X_{1}^{\prime \ell}+\Gamma_{2}^{\ell}\right) \\
& =H\left(W_{1} \mid Y_{1}^{\ell}\right) \leq \ell \varepsilon_{\ell}
\end{aligned}
$$

where $\varepsilon_{\ell} \rightarrow 0$ as $\ell$ grows. We have used the invertibility property of the deterministic multiple access channel in (57), and (58) follows from the Fano's inequality, and the fact that 
$D_{1}$ can decode the message sent by $S_{1}$. Therefore, we have $H\left(\Gamma_{1}^{\ell} \mid Y_{1}^{\prime \ell}, \Gamma_{2}^{\ell}\right) \leq \ell \varepsilon_{\ell}$. Hence

$$
\begin{aligned}
H\left(Y_{2}^{\prime \ell} \mid Y_{1}^{\prime \ell}, \Gamma_{2}^{\ell}\right) & \leq H\left(Y_{2}^{\prime \ell}, \Gamma_{1}^{\ell} \mid Y_{1}^{\prime \ell}, \Gamma_{2}^{\ell}\right) \\
& =H\left(Y_{2}^{\prime \ell} \mid \Gamma_{1}^{\ell}, Y_{1}^{\prime \ell}, \Gamma_{2}\right)+H\left(\Gamma_{1}^{\ell} \mid Y_{1}^{\prime \ell}, \Gamma_{2}^{\ell}\right) \\
& \leq H\left(Y_{2}^{\prime \ell} \mid \Gamma_{1}^{\ell}\right)+\ell \varepsilon_{\ell} \\
& =H\left(\mathbf{M}_{22} X_{2}^{\ell} \mid \mathbf{M}_{12} X_{2}^{\ell}\right)+\ell \varepsilon_{\ell} \\
& \leq \ell\left(m_{22}-m_{12}\right)^{+}+\ell \varepsilon_{\ell}
\end{aligned}
$$

where the derivation of the last inequality above is based on Lemma 4 and very similar to that of (39), and hence, we skip it here. Replacing the upper bound for each term in (56), we get

$$
R_{1}+R_{2} \leq \max \left(m_{11}, m_{12}\right)+n_{12}+\left(m_{22}-m_{12}\right)^{+} .
$$

It is worth mentioning that the cut-set bound for $\Omega_{s}=\left\{S_{1}, S_{2}\right\}$ and $\Omega_{d}=\left\{A, B, D_{1}, D_{2}\right\}$ gives us

$$
R_{1}+R_{2} \leq \max \left(m_{11}+m_{22}, m_{12}\right)
$$

which is looser than the genie-aided bound.

(DZZ-6) $R_{1}+R_{2} \leq \max \left(n_{11}, n_{12}\right)+\left(n_{22}-n_{12}\right)^{+}+m_{12}$ : The last inequality captures the maximum flow of information from the relays to the destinations. Intuitively, this inequality says that the number of interfering bits can get neutralized at $D_{1}$ cannot exceed the minimum of $m_{12}$ and $n_{12}$. In order to make this intuition formal, we provide $\Gamma_{1}^{\ell}$, the partial information about $W_{2}$ which is available at $A$, as side information for $D_{1}$. We then have

$$
\begin{aligned}
\ell\left(R_{1}+R_{2}\right) & \leq I\left(Y_{1}^{\ell}, Y_{2}^{\ell} ; X_{1}^{\ell}, X_{2}^{\ell}\right)=H\left(Y_{1}^{\ell}, Y_{2}^{\ell}\right) \\
& \leq H\left(Y_{1}^{\ell}, Y_{2}^{\ell}, \Gamma_{1}^{\ell}\right) \\
& \leq H\left(Y_{1}^{\ell}\right)+H\left(\Gamma_{1}^{\ell}\right)+H\left(Y_{2}^{\ell} \mid Y_{1}^{\ell}, \Gamma_{1}^{\ell}\right) .
\end{aligned}
$$

Again, we can simply upper bound the first two terms by the rank of the corresponding matrices. In order to bound the last term, similar to the proof of (DZZ-5), we use the following bounding technique:

$$
\begin{aligned}
H\left(\Gamma_{2}^{\ell} \mid Y_{1}^{\ell}, \Gamma_{1}^{\ell}\right) & =H\left(Y_{1}^{\ell}-\mathbf{N}_{11} X_{1}^{\prime \ell} \mid Y_{1}^{\ell}, \Gamma_{1}^{\ell}\right) \\
& \leq H\left(X_{1}^{\prime \ell} \mid Y_{1}^{\ell}, \Gamma_{1}^{\ell}\right) \\
& \leq H\left(Y_{1}^{\prime \ell} \mid Y_{1}^{\ell}, \Gamma_{1}^{\ell}\right) \\
& =H\left(\mathbf{M}_{11} X_{1}^{\ell}+\Gamma_{1}^{\ell} \mid Y_{1}^{\ell}, \Gamma_{1}^{\ell}\right) \\
& \leq H\left(X_{1}^{\ell} \mid Y_{1}^{\ell}, \Gamma_{1}^{\ell}\right) \\
& \leq H\left(X_{1}^{\ell} \mid Y_{1}^{\ell}\right) \\
& \leq H\left(W_{1} \mid Y_{1}^{\ell}\right) \leq \ell \varepsilon_{\ell},
\end{aligned}
$$

where (62) follows from the Fano's inequality. This inequality can be used as

$$
\begin{aligned}
H\left(Y_{2}^{\ell} \mid Y_{1}^{\ell}, \Gamma_{1}^{\ell}\right) & \leq H\left(Y_{2}^{\ell}, \Gamma_{2}^{\ell} \mid Y_{1}^{\ell}, \Gamma_{1}^{\ell}\right) \\
& =H\left(Y_{2}^{\ell} \mid \Gamma_{2}^{\ell}, Y_{1}^{\ell}, \Gamma_{1}^{\ell}\right)+H\left(\Gamma_{2}^{\ell} \mid Y_{1}^{\ell}, \Gamma_{1}^{\ell}\right) \\
& \leq H\left(Y_{2}^{\ell} \mid \Gamma_{2}^{\ell}\right)+\ell \varepsilon_{\ell} \\
& =H\left(\mathbf{N}_{22} X_{2}^{\prime \ell} \mid \mathbf{N}_{12} X_{2}^{\prime \ell}\right)+\ell \varepsilon_{\ell} \\
& \leq \ell\left(n_{22}-n_{12}\right)^{+}+\ell \varepsilon_{\ell} .
\end{aligned}
$$

We again used Lemma 4 in the last inequality. Now, we have

$$
R_{1}+R_{2} \leq \max \left(n_{11}, n_{12}\right)+m_{12}+\left(n_{22}-n_{12}\right)^{+} .
$$

Again, it is easy to show that this bound is tighter than the cut-set bound for $\Omega_{s}=\left\{S_{1}, S_{2}, A, B\right\}$ and $\Omega_{d}=\left\{D_{1}, D_{2}\right\}$

$$
R_{1}+R_{2} \leq \max \left(n_{11}+n_{22}, n_{12}\right) .
$$

This completes the proof of the converse part of Theorem 3.

\section{B. Achievability Proof}

In this part we will show that all rate pairs satisfying inequalities (DZZ-1)-(DZZ-6) are achievable. In particular, we introduce a coding scheme which achieves such rates. Our coding strategy provides the interference neutralization at the destination. This is performed by splitting the messages into two parts, namely private and functional parts. The private sub-messages can be decoded at the relays, and forwarded to the destinations. The functional sub-message of the second source can be also decoded at $B$. However, $A$ only receives a combination (xor) of the functional sub-messages, and cannot decode them. It only forwards such combination on proper (power) levels such that the interference caused by the functional sub-message of $S_{2}$ get neutralized over the second layer of the network, and $D_{1}$ can decode the sub-message of its interest.

Our analysis is based on characterizing the number of pure and combined bits can be sent through each layer of the network. In the following we focus on one layer of the network, and obtain an achievable rate region for these numbers. Next, we use this region to build the encoding scheme for the $\mathrm{ZZ}$ network, and obtain an achievable rate region, which matches with the outer bound.

Definition 4: Consider a deterministic Z network, with gains $\left(n_{11}, n_{12}, n_{22}\right)$. as shown in Fig. 14. Each of the transmitters has a set of information bits to transmit to the receivers. This set for $F_{i}$ includes $\Upsilon_{i}$ private bits and $\Upsilon_{0}$ functional bits, namely, $\mathcal{W}_{i, P}=\left\{W_{i, P}(1), \ldots, W_{i, P}\left(\Upsilon_{i}\right)\right\}$ and $\mathcal{W}_{i, N}=\left\{W_{i, N}(1), \ldots, X_{i, N}\left(\Upsilon_{0}\right)\right\}$. The second receiver wishes to receive all the private and functional bits of $F_{2}$, while the first receiver is interested in receiving the private bits of $F_{1}$, and the xor of the functional bits of $F_{1}$ and $F_{2}$. More precisely, denoting by $\hat{\mathcal{W}}_{i}$ the set of bits $G_{i}$ is interested in, we have

$\hat{\mathcal{W}}_{1}=\mathcal{W}_{1, P} \cup\left\{\tilde{W}_{1, N}(j) \triangleq W_{1, N}(j) \oplus W_{2, N}(j): j=1, \ldots, \Upsilon_{0}\right\}$ $\hat{\mathcal{W}}_{2}=\mathcal{W}_{2, P} \cup \mathcal{W}_{2, N}$.

We term this network with the described decoding demands as deterministic Z-neutralization network. The goal is to characterize the set achievable tuples $\left(\Upsilon_{0}, \Upsilon_{1}, \Upsilon_{2}\right)$ for the deterministic Z-neutralization network.

The following lemma gives an achievable rate region for the deterministic Z-neutralization network. The proof of this lemma can be found in Appendix C. 


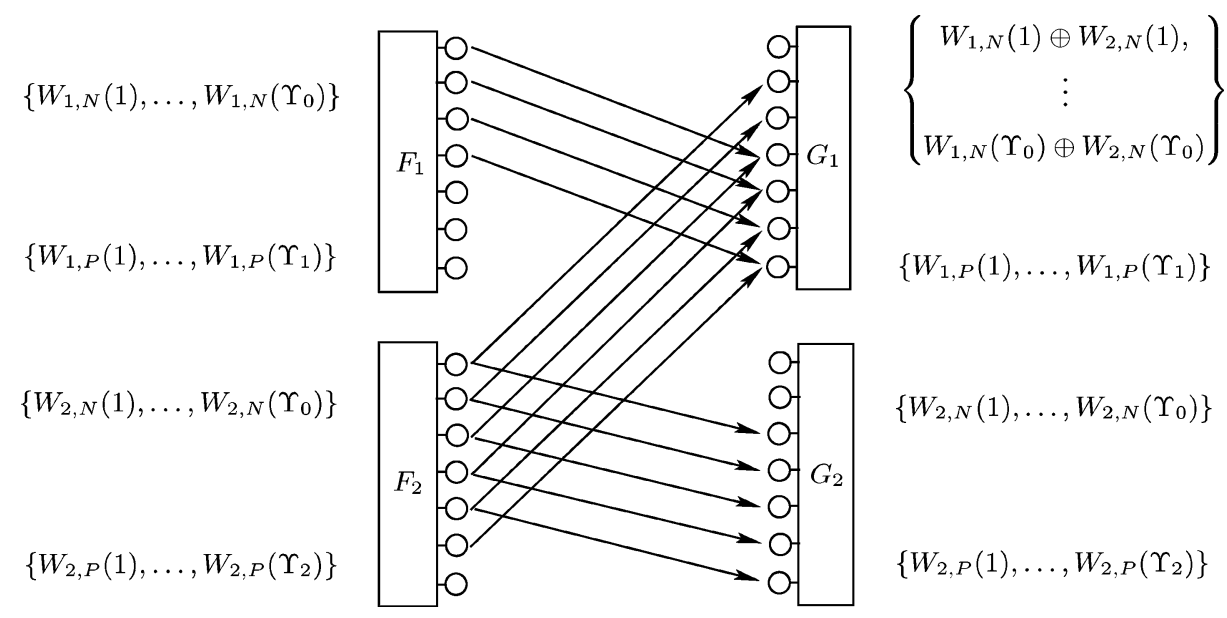

Fig. 14. Deterministic Z-neutralization network with the message demands.

Lemma 6: Consider the deterministic Z-neutralization network defined in Definition 4 with channel gains $\left(n_{11}, n_{12}, n_{22}\right)$ (see Fig. 14). Any rate tuple $\left(\Upsilon_{0}, \Upsilon_{1}, \Upsilon_{2}\right)$ satisfying

$$
\begin{aligned}
\Upsilon_{0} & \leq \lambda \triangleq \min \left\{n_{11}, n_{12}, n_{22}\right\} \\
\Upsilon_{0}+\Upsilon_{1} & \leq n_{11} \\
\Upsilon_{0}+\Upsilon_{2} & \leq n_{22} \\
\Upsilon_{0}+\Upsilon_{1}+\Upsilon_{2} & \leq \mu \triangleq \max \left\{n_{11}, n_{12}, n_{22}, n_{11}+n_{22}-n_{12}\right\}
\end{aligned}
$$

is achievable for this network.

Now, having an achievable rate region for the deterministic Z-neutralization network, we are ready to present the coding scheme and analyze its rate region for the $\mathrm{ZZ}$ network.

Recall that the ZZ network consists of two cascaded Z network. In first layer, the source nodes split their message into private and functional parts. They can send these parts to the relays as long as their rates belong to the achievable rate region of the first layer given in Lemma 6 . Once the relays receive these sub-messages, forward them to the destination nodes using the same scheme for the private and functional sub-messages. This can be done if the rate tuple for the sub-messages satisfy the corresponding inequalities for the second layer as well. Note that functional bits received at the destination are

$$
\begin{aligned}
\tilde{W}_{1, N}(j) \oplus W_{2, N}(j) & \\
& =\left[W_{1, N}(j) \oplus W_{2, N}(j)\right] \oplus W_{2, N}(j)=W_{1, N}(j) .
\end{aligned}
$$

Therefore, the interference of these bits get neutralized, and pure information bits will be received at the destination.

The achievable rate region of this scheme is given by

$$
\begin{aligned}
& \mathcal{R}_{\mathrm{ach}}^{\mathrm{DZZ}}=\left\{\left(R_{1}, R_{2}\right)\right.: \exists \Upsilon_{0}, \Upsilon_{1}, \Upsilon_{2} \geq 0 \\
& R_{1}=\Upsilon_{0}+\Upsilon_{1} \\
& R_{2}=\Upsilon_{0}+\Upsilon_{2} \\
& \Upsilon_{0} \leq \min \left\{\lambda_{m}, \lambda_{n}\right\} \\
& \Upsilon_{0}+\Upsilon_{1} \leq \min \left\{m_{11}, n_{11}\right\} \\
& \Upsilon_{0}+\Upsilon_{2} \leq \min \left\{m_{22}, n_{22}\right\} \\
&\left.\Upsilon_{0}+\Upsilon_{1}+\Upsilon_{2} \leq \min \left\{\mu_{m}, \mu_{n}\right\}\right\} .
\end{aligned}
$$

Here we used subscripts $m$ and $n$ to denote $\lambda$ and $\mu$ parameters for the first and the second layers of the network, respectively. Applying Fourier-Motzkin elimination [1] on this set to project it on the $\left(R_{1}, R_{2}\right)$ plane gives us the rate region claimed in the theorem.

\section{DISCUSSION}

Interference management is perhaps the most fundamental open problem in wireless networks. The recent progress in (approximate) characterization of the interference channel capacity and the utility of the deterministic approach inspired the questions studied in this paper. Even though the interference-relay networks studied in this work were special, they revealed several new features needed for information transmission. In particular, the interference neutralization and network flow decomposition techniques were uncovered through the study of ZZ and ZS networks. The main idea behind this technique is to reduce the power of an interfering signal received at a destination through different paths over the network. This is done by processing the received signal at the relays in a way such that the total effective interference power becomes negligible. Note that even if a complete neutralization is impossible, this technique can be still used to reduce the interference power, and hence improve the transmission rates. Moreover, we believe that the neutralization technique is robust to channel uncertainties and one could get partial neutralization in such situations. Although it is only illustrated for the ZZ network in this paper, we believe that the technique can be generalized to larger networks. This is a topic of ongoing work.

We also saw the importance of using structured lattice codes for interference neutralization. This result suggests that, unlike the single unicast or multicast networks for which using random codes is almost optimal, more structured codes are required when there are more than one flow of information in the network, which have to be delivered to different receivers.

We also believe that the outer bounding techniques developed in this work could have more general applicability in the wireless multiple-unicast problem. The two-unicast problem in arbitrary layered wireless networks would be a natural next step arising out of our work. The deterministic approach for this problem has already provided some interesting new techniques 


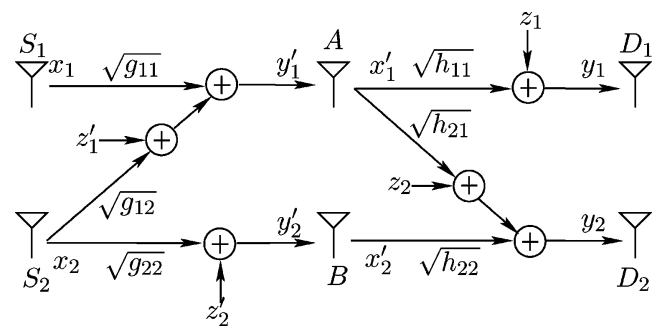

Fig. 15. Gaussian ZS network.

[19]. In summary we believe that the deterministic approach is a promising methodology to make progress on the wireless multiple-unicast problem.

\section{APPENDIX A}

GAUSSIAN ZS NETWORK

A) Outer Bound: In the following we will prove each of the inequalities in (GZS-1)-(GZS-10), separately. We will use the notation as shown in Fig. 15, and assume that the rate pair $\left(R_{1}, R_{2}\right)$ can be achieved with small enough decoding error probability using a code of length $\ell$.

Lemma 7: Any achievable rate pair $\left(R_{1}, R_{2}\right)$ satisfies

$$
\begin{aligned}
\ell R_{1} & \leq I\left(x_{1}^{\ell} ; y_{1}^{\ell}\right)+\ell \varepsilon_{\ell} \\
\ell R_{2} & \leq I\left(x_{2}^{\ell} ; y_{2}^{\ell}\right)+\ell \varepsilon_{\ell} \\
\ell\left(R_{1}+R_{2}\right) & \leq I\left(x_{1}^{\ell}, x_{2}^{\ell} ; y_{1}^{\ell}, y_{2}^{\ell}\right)+\ell \varepsilon_{\ell} .
\end{aligned}
$$

Note that $\varepsilon_{\ell} \rightarrow 0$ as $\ell$ grows.

This lemma is a consequence of the Fano's lemma combined with the decodability requirements imposed by the problem, and its proof is given in Appendix C.

Most of the inequalities in (GZS-1)-(GZS-10) are cut-set type bounds, although the proof presented here is slightly different than the standard argument. However, the sum-rate bounds in (GZS-3) and (GZS-10) are different from the well known cut-set bounds. These two bounds are in general tighter than the cut values for the corresponding cuts. This is because in deriving a cut-set bound, the decoders are inherently allowed to cooperate, while individual decoding abilities are imposed in this problem. In the following we first present the proofs of (GZS-3) and (GZS-10), which are more involved, and then prove the cut-set type bounds.

\section{1) Proofs of Non-Cut-Set Type Bounds}

- (GZS-3) $R_{1}+R_{2}<\frac{1}{2} \log \left(1+g_{11}+g_{12}\right)+$ $\frac{1}{2} \log \left(1+\frac{g_{22}}{g_{12}}\right):$ We start with Lemma 7 for the sum-rate which implies

$$
\begin{aligned}
\ell\left(R_{1}+R_{2}\right) \leq & I\left(x_{1}^{\ell}, x_{2}^{\ell} ; y_{1}^{\ell}, y_{2}^{\ell}\right)+\ell \varepsilon_{\ell} \\
\leq & I\left(x_{1}^{\ell}, x_{2}^{\ell} ; y_{1}^{\prime \ell}, y_{2}^{\prime \ell}\right)+\ell \varepsilon_{\ell} \\
= & I\left(x_{1}^{\ell}, x_{2}^{\ell} ; y_{1}^{\prime \ell}\right)+I\left(x_{1}^{\ell}, x_{2}^{\ell} ; y_{2}^{\prime \ell} \mid y_{1}^{\prime \ell}\right)+\ell \varepsilon_{\ell} \\
\leq & \frac{\ell}{2} \log \left(1+g_{11}+g_{12}\right)+h\left(y_{2}^{\prime \ell} \mid y_{1}^{\prime \ell}\right) \\
& -h\left(y_{2}^{\prime \ell} \mid y_{1}^{\prime \ell}, x_{1}^{\ell}, x_{2}^{\ell}\right)+\ell \varepsilon_{\ell}
\end{aligned}
$$

where (A.4) follows from the data processing inequality. Now, note that

$$
h\left(y_{2}^{\prime \ell} \mid y_{1}^{\prime \ell}, x_{1}^{\ell}, x_{2}^{\ell}\right)=h\left(z_{2}^{\prime \ell}\right)=\frac{\ell}{2} \log (2 \pi e)
$$

Moreover

$$
\begin{aligned}
h\left(y_{2}^{\prime \ell}, W_{1} \mid y_{1}^{\prime \ell}\right) & =h\left(y_{2}^{\prime \ell} \mid y_{1}^{\prime \ell}\right)+H\left(W_{1} \mid y_{1}^{\prime \ell}, y_{2}^{\prime \ell}\right) \\
& =H\left(W_{1} \mid y_{1}^{\prime \ell}\right)+h\left(y_{2}^{\prime \ell} \mid W_{1}, y_{1}^{\prime \ell}\right) \\
& \leq H\left(W_{1} \mid y_{1}^{\ell}\right)+h\left(y_{2}^{\prime \ell} \mid W_{1}, y_{1}^{\prime \ell}\right) .
\end{aligned}
$$

Therefore

$$
\begin{aligned}
h\left(y_{2}^{\prime \ell} \mid y_{1}^{\prime \ell}\right) \leq & h\left(y_{2}^{\prime \ell} \mid W_{1}, y_{1}^{\prime \ell}\right)+\ell \varepsilon_{\ell} \\
\leq & h\left(y_{2}^{\prime \ell} \mid x_{1}^{\ell}, y_{1}^{\prime \ell}\right)+\ell \varepsilon_{\ell} \\
= & h\left(y_{2}^{\prime \ell} \mid x_{1}^{\ell}, \sqrt{g_{12}} x_{2}^{\ell}+z_{1}^{\prime \ell}\right)+\ell \varepsilon_{\ell} \\
\leq & h\left(\sqrt{g_{22}} x_{2}^{\ell}+z_{2}^{\prime \ell} \mid \sqrt{g_{12}} x_{2}^{\ell}+z_{1}^{\prime \ell}\right)+\ell \varepsilon_{\ell} \\
= & h\left(\sqrt{g_{22}} x_{2}^{\ell}+z_{2}^{\prime \ell}\right. \\
& \left.-\frac{\sqrt{g_{22}}}{\sqrt{g_{12}}}\left(\sqrt{g_{12}} x_{2}^{\ell}+z_{1}^{\prime \ell}\right) \mid \sqrt{g_{12}} x_{2}^{\ell}+z_{1}^{\prime \ell}\right)+\ell \varepsilon_{\ell} \\
\leq & h\left(z_{2}^{\prime \ell}-\frac{\sqrt{g_{22}}}{\sqrt{g_{12}}} z_{1}^{\prime \ell}\right)+\ell \varepsilon_{\ell} \\
= & \frac{\ell}{2} \log (2 \pi e)\left(1+\frac{g_{22}}{g_{12}}\right)+\ell \varepsilon_{\ell}
\end{aligned}
$$

where (A.6) holds since $x_{1}^{\ell}$ is a function of $W_{1}$, and in (A.7) we used the invertibility property of the function $y_{1}^{\ell}=$ $\sqrt{g_{11}} x_{1}^{\ell}+\sqrt{g_{12}} x_{2}^{\ell}+z_{1}^{\prime \ell}$. Replacing $h\left(y_{2}^{\prime \ell} \mid y_{1}^{\prime \ell}\right)$ from (A.8) in (A.5), we get the desired bound.

- (GZS-10) $R_{1}+R_{2}<\frac{1}{2} \log \left(1+\frac{h_{11}}{h_{21}}\right)+\frac{\ell}{2} \log \left(1+h_{21}+\right.$ $\left.h_{22}+2 \sqrt{h_{21} h_{22}}\right)$ : The sum-rate can be upper bounded as in Lemma 7. Next, we have

$$
\begin{aligned}
\ell\left(R_{1}+R_{2}\right) \leq & I\left(x_{1}^{\ell}, x_{2}^{\ell} ; y_{1}^{\ell}, y_{2}^{\ell}\right)+\ell \varepsilon_{\ell} \\
\leq & I\left(x_{1}^{\prime \ell}, x_{2}^{\prime \ell} ; y_{1}^{\ell}, y_{2}^{\ell}\right)+\ell \varepsilon_{\ell} \\
= & I\left(x_{1}^{\prime \ell}, x_{2}^{\prime \ell} ; y_{2}^{\ell}\right)+I\left(x_{2}^{\prime \ell} ; y_{1}^{\ell} \mid y_{2}^{\ell}\right) \\
& +I\left(x_{1}^{\prime \ell} ; y_{1}^{\ell} \mid x_{2}^{\prime \ell}, y_{2}^{\ell}\right)+\ell \varepsilon_{\ell} .
\end{aligned}
$$

The first term in (A.9) can be simply upper bounded as

$I\left(x_{1}^{\prime \ell}, x_{2}^{\prime \ell} ; y_{2}^{\ell}\right) \leq \frac{\ell}{2} \log \left(1+h_{21}+h_{22}+2 \sqrt{h_{21} h_{22}}\right)$.

In order to bound the second term, we can use the fact that $W_{2}$ can be decoded from $y_{2}^{\ell}$, and write

$$
\begin{aligned}
I\left(x_{2}^{\prime \ell} ; y_{1}^{\ell}, W_{2} \mid y_{2}^{\ell}\right) & =I\left(x_{2}^{\prime \ell} ; y_{1}^{\ell} \mid y_{2}^{\ell}\right)+I\left(x_{2}^{\prime \ell} ; W_{2} \mid y_{1}^{\ell}, y_{2}^{\ell}\right) \\
& =I\left(x_{2}^{\prime \ell} ; y_{1}^{\ell} \mid W_{2}, y_{2}^{\ell}\right)+I\left(x_{2}^{\prime \ell} ; W_{2} \mid y_{2}^{\ell}\right) \\
& \leq I\left(x_{2}^{\prime \ell} ; y_{1}^{\ell} \mid W_{2}, y_{2}^{\ell}\right)+H\left(W_{2} \mid y_{2}^{\ell}\right) \\
& \leq I\left(x_{2}^{\prime \ell} ; y_{1}^{\ell} \mid y_{2}^{\ell}, W_{2}\right)+\ell \varepsilon_{\ell} .
\end{aligned}
$$

Therefore

$$
\begin{aligned}
I\left(x_{2}^{\prime \ell} ; y_{1}^{\ell} \mid y_{2}^{\ell}\right) & \leq I\left(x_{2}^{\prime \ell} ; y_{1}^{\ell} \mid y_{2}^{\ell}, W_{2}\right)+\ell \varepsilon_{\ell} \\
& \leq I\left(y_{2}^{\prime \ell} ; y_{1}^{\ell} \mid y_{2}^{\ell}, W_{2}\right)+\ell \varepsilon_{\ell}=\ell \varepsilon_{\ell}
\end{aligned}
$$


where the second inequality follows from the fact that $x_{2}^{\prime \ell}$ is a function of $y_{2}^{\prime \ell}$, and (A.11) holds since $y_{2}^{\prime \ell}$ and $y_{1}^{\ell}$ are independent if $W_{2}$ is given.

Finally, we bound the last term as follows:

$$
\begin{aligned}
& I\left(x_{1}^{\prime \ell} ; y_{1}^{\ell} \mid x_{2}^{\prime \ell}, y_{2}^{\ell}\right) \\
&= I\left(x_{1}^{\prime \ell} ; y_{1}^{\ell} \mid x_{2}^{\prime \ell}, \sqrt{h_{21}} x_{1}^{\prime \ell}+z_{2}^{\ell}\right) \\
&= h\left(\sqrt{h_{11}} x_{1}^{\prime \ell}+z_{1}^{\ell} \mid x_{2}^{\prime \ell}, \sqrt{h_{21}} x_{1}^{\prime \ell}+z_{2}^{\ell}\right) \\
&-h\left(y_{1}^{\ell} \mid x_{2}^{\prime \ell}, \sqrt{h_{21}} x_{1}^{\prime \ell}+z_{2}^{\ell}, x_{1}^{\prime \ell}\right) \\
& \leq h\left(\sqrt{h_{11}} x_{1}^{\prime \ell}+z_{1}^{\ell}-\frac{\sqrt{h_{11}}}{\sqrt{h_{21}}}\left(\sqrt{h_{21}} x_{1}^{\prime \ell}+z_{2}^{\ell}\right)\right)-h\left(z_{1}^{\ell}\right) \\
& \leq \frac{\ell}{2} \log \left(1+\frac{h_{11}}{h_{12}}\right) .
\end{aligned}
$$

Replacing the bound derived for the three terms, (A.10), (A.11), and (A.12) in (A.9), we get the desired bound.

\section{2) Proofs of Cut-Set Type Bounds}

- (GZS-1) $R_{1}<\frac{1}{2} \log \left(1+g_{11}\right)$ : We start by Lemma 7, and write

$$
\begin{aligned}
\ell R_{1} & =I\left(x_{1}^{\ell} ; y_{1}^{\ell}\right)+\ell \varepsilon_{\ell} \\
& \leq I\left(x_{1}^{\ell} ; y_{1}^{\prime \ell}\right)+\ell \varepsilon_{\ell} \\
& \leq I\left(x_{1}^{\ell} ; x_{2}^{\ell}, y_{1}^{\prime \ell}\right)+\ell \varepsilon_{\ell} \\
& =I\left(x_{1}^{\ell} ; x_{2}^{\ell}\right)+I\left(x_{1}^{\ell} ; y_{1}^{\prime \ell} \mid x_{2}^{\ell}\right)+\ell \varepsilon_{\ell} \\
& \leq \frac{\ell}{2} \log \left(1+g_{11}\right)+\ell \varepsilon_{\ell}
\end{aligned}
$$

where (A.13) follows from the data-processing inequality for the Markov chain $x_{1}^{\ell} \leftrightarrow y_{1}^{\ell \ell} \leftrightarrow x_{1}^{\prime \ell} \leftrightarrow y_{1}^{\ell}$, and in (A.14) we used the fact that $x_{1}^{\ell}$ and $x_{2}^{\ell}$ are independent. It is worth mentioning that this inequality essentially bounds the maximum flow that can be transmitted through the cut $\Omega_{s}=\left\{S_{1}\right\}$ and $\Omega_{d}=\left\{S_{2}, A, B, D_{1}, D_{2}\right\}$.

- (GZS-2) $R_{2}<\frac{1}{2} \log \left(1+g_{12}+g_{22}\right)$ : Again starting from Lemma 7, we have

$$
\begin{aligned}
\ell R_{2} & \leq I\left(x_{2}^{\ell} ; y_{2}^{\ell}\right)+\ell \varepsilon_{\ell} \\
& \leq I\left(x_{2}^{\ell} ; y_{1}^{\prime \ell}, y_{2}^{\prime \ell}\right)+\ell \varepsilon_{\ell} \\
& \leq I\left(x_{2}^{\ell} ; x_{1}^{\ell}, y_{1}^{\prime \ell}, y_{2}^{\prime \ell}\right)+\ell \varepsilon_{\ell} \\
& =I\left(x_{2}^{\ell} ; x_{1}^{\ell}\right)+I\left(x_{2}^{\ell} ; y_{1}^{\prime \ell}, y_{2}^{\prime \ell} \mid x_{1}^{\ell}\right)+\ell \varepsilon_{\ell} \\
& =h\left(y_{1}^{\prime \ell}, y_{2}^{\prime \ell} \mid x_{1}^{\ell}\right)-h\left(y_{1}^{\prime \ell}, y_{2}^{\prime \ell} \mid x_{1}^{\ell}, x_{2}^{\ell}\right)+\ell \varepsilon_{\ell} \\
& \leq h\left(\sqrt{g_{12}} x_{2}^{\ell}+z_{1}^{\prime \ell}, \sqrt{g_{22}} x_{2}^{\ell}+z_{2}^{\prime \ell}\right)-h\left(z_{1}^{\prime \ell}, z_{2}^{\prime \ell}\right)+\ell \varepsilon_{\ell} \\
& \leq \frac{\ell}{2} \log \left(1+g_{12}+g_{22}\right)+\ell \varepsilon_{\ell}
\end{aligned}
$$

where the data processing inequality implies (A.16) for the Markov chain $x_{2}^{\ell} \leftrightarrow\left(y_{1}^{\prime \ell}, y_{2}^{\prime \ell}\right) \leftrightarrow\left(x_{1}^{\prime \ell}, x_{2}^{\prime \ell}\right) \leftrightarrow y_{1}^{\ell}$. Note that this bound is essentially the cut-set bound for the cut $\Omega_{s}=\left\{S_{2}\right\}$ and $\Omega_{d}=\left\{S_{1}, A, B, D_{1}, D_{2}\right\}$.
- (GZS-4) $R_{2}<\frac{1}{2} \log \left(1+g_{12}\right)+\frac{1}{2} \log \left(1+h_{22}\right)$ : Again we use Lemma 7 to upper bound $R_{2}$ as

$$
\begin{aligned}
\ell R_{2} \leq & I\left(x_{2}^{\ell} ; y_{2}^{\ell}\right)+\ell \varepsilon_{\ell} \\
\leq & I\left(x_{2}^{\prime \ell}, x_{2}^{\ell} ; x_{1}^{\ell}, y_{1}^{\prime \ell}, y_{2}^{\ell}\right)+\ell \varepsilon_{\ell} \\
= & I\left(x_{2}^{\prime \ell}, x_{2}^{\ell} ; x_{1}^{\ell}\right)+I\left(x_{2}^{\prime \ell}, x_{2}^{\ell} ; y_{1}^{\prime \ell}, y_{2}^{\ell} \mid x_{1}^{\ell}\right)+\ell \varepsilon_{\ell} \\
= & I\left(x_{2}^{\prime \ell}, x_{2}^{\ell} ; y_{1}^{\prime \ell} \mid x_{1}^{\ell}\right)+I\left(x_{2}^{\prime \ell}, x_{2}^{\ell} ; y_{2}^{\ell} \mid x_{1}^{\ell}, y_{1}^{\prime \ell}\right)+\ell \varepsilon_{\ell} \\
= & I\left(x_{2}^{\ell} ; y_{1}^{\prime \ell} \mid x_{1}^{\ell}\right)+I\left(x_{2}^{\prime \ell} ; y_{1}^{\prime \ell} \mid x_{1}^{\ell}, x_{2}^{\ell}\right)+I\left(x_{2}^{\prime \ell} ; y_{2}^{\ell} \mid x_{1}^{\ell}, y_{1}^{\prime \ell}\right) \\
& +I\left(x_{2}^{\ell} ; y_{2}^{\ell} \mid x_{1}^{\ell}, y_{1}^{\prime \ell}, x_{2}^{\prime \ell}\right)+\ell \varepsilon_{\ell} \\
= & I\left(x_{2}^{\ell} ; y_{1}^{\prime \ell} \mid x_{1}^{\ell}\right)+I\left(x_{2}^{\prime \ell} ; y_{2}^{\ell} \mid x_{1}^{\ell}, y_{1}^{\prime \ell}\right)+\ell \varepsilon_{\ell} \\
\leq & \frac{\ell}{2} \log \left(1+g_{12}\right)+\frac{\ell}{2} \log \left(1+h_{22}\right)+\ell \varepsilon_{\ell} .
\end{aligned}
$$

Note that we used the fact that the second and fourth terms in (A.18) are zero. This follows from:

$$
\begin{aligned}
I\left(x_{2}^{\prime \ell} ; y_{1}^{\prime \ell} \mid x_{1}^{\ell}, x_{2}^{\ell}\right) & \leq I\left(x_{2}^{\prime \ell} ; y_{1}^{\prime \ell}-\sqrt{g_{11}} x_{1}^{\ell}-\sqrt{g_{12}} x_{2}^{\ell} \mid x_{1}^{\ell}, x_{2}^{\ell}\right) \\
& =I\left(x_{2}^{\prime \ell} ; z_{1}^{\prime \ell} \mid x_{1}^{\ell}, x_{2}^{\ell}\right)=0
\end{aligned}
$$

and

$$
\begin{aligned}
& I\left(x_{2}^{\ell} ; y_{2}^{\ell} \mid x_{1}^{\ell}, y_{1}^{\prime \ell}, x_{2}^{\prime \ell}\right) \\
& \quad \leq I\left(x_{2}^{\ell} ; y_{2}^{\ell} \mid x_{1}^{\ell}, x_{1}^{\prime \ell}, x_{2}^{\prime \ell}\right) \\
& \quad \leq I\left(x_{2}^{\ell} ; y_{2}^{\ell}-\sqrt{h_{21}} x_{1}^{\prime \ell}-\sqrt{h_{22}} x_{2}^{\prime \ell} \mid x_{1}^{\ell}, x_{1}^{\prime \ell}, x_{2}^{\prime \ell}\right) \\
& \quad \leq I\left(x_{2}^{\ell} ; z_{2}^{\ell} \mid x_{1}^{\ell}, x_{1}^{\prime \ell}, x_{2}^{\prime \ell}\right)=0 .
\end{aligned}
$$

- (GZS-5) $R_{1}+R_{2}<\frac{1}{2} \log \left(1+g_{22}\right)+\frac{1}{2} \log \left(1+h_{11}+h_{21}\right)$ : We start from Lemma 7 and write

$$
\begin{aligned}
\ell( & \left.R_{1}+R_{2}\right) \\
\leq & I\left(y_{1}^{\ell}, y_{2}^{\ell} ; x_{1}^{\ell}, x_{2}^{\ell}\right)+\ell \varepsilon_{\ell} \\
\leq & I\left(y_{1}^{\ell}, y_{2}^{\ell} ; x_{1}^{\prime \ell}, x_{2}^{\ell}\right)+\ell \varepsilon_{\ell} \\
\leq & I\left(y_{1}^{\ell}, y_{2}^{\ell}, y_{2}^{\prime \ell} ; x_{1}^{\prime \ell}, x_{2}^{\ell}\right)+\ell \varepsilon_{\ell} \\
= & I\left(y_{2}^{\prime \ell} ; x_{2}^{\ell}\right)+I\left(y_{2}^{\prime \ell} ; x_{1}^{\prime \ell} \mid x_{2}^{\ell}\right)+I\left(y_{1}^{\ell}, y_{2}^{\ell} ; x_{1}^{\prime \ell}, x_{2}^{\ell} \mid y_{2}^{\prime \ell}\right)+\ell \varepsilon_{\ell} \\
= & I\left(y_{2}^{\prime \ell} ; x_{2}^{\ell}\right)+I\left(y_{1}^{\ell}, y_{2}^{\ell} ; x_{1}^{\prime \ell}, x_{2}^{\ell} \mid x_{2}^{\prime \ell}\right)+\ell \varepsilon_{\ell} \\
= & I\left(y_{2}^{\prime \ell} ; x_{2}^{\ell}\right)+h\left(y_{1}^{\ell}, y_{2}^{\ell} \mid x_{2}^{\prime \ell}\right)-h\left(y_{1}^{\ell}, y_{2}^{\ell} \mid x_{2}^{\ell}, x_{1}^{\prime \ell}, x_{2}^{\prime \ell}\right)+\ell \varepsilon_{\ell} \\
\leq & I\left(y_{2}^{\prime \ell} ; x_{2}^{\ell}\right)+h\left(\sqrt{h_{11}} x_{1}^{\prime \ell}+z_{1}^{\ell}, \sqrt{h_{21}} x_{1}^{\prime \ell}+z_{2}^{\ell}\right) \\
& -h\left(z_{1}^{\ell}, z_{2}^{\ell}\right)+\ell \varepsilon_{\ell} \\
\leq & \frac{\ell}{2} \log \left(1+g_{22}\right)+\frac{\ell}{2} \log \left(1+h_{11}+h_{21}\right)+\ell \varepsilon_{\ell}
\end{aligned}
$$

where (A.20) follows from the data processing inequality for the Markov chain $\left(x_{1}^{\ell}, x_{2}^{\ell}\right) \leftrightarrow\left(y_{1}^{\ell \ell}, y_{2}^{\prime \ell}\right) \leftrightarrow$ $\left(x_{1}^{\prime \ell}, x_{2}^{\prime \ell}\right) \leftrightarrow\left(y_{1}^{\ell}, y_{2}^{\ell}\right)$. Note that this bound essentially captures the maximum flow of information through the cut $\Omega_{s}=\left\{S_{1}, S_{2}, A\right\}$ and $\Omega_{d}=\left\{B, D_{1}, D_{2}\right\}$. 
- (GZS-6) $R_{1}+R_{2}<\frac{1}{2} \log \left(1+g_{11}+g_{12}\right)+\frac{1}{2} \log \left(1+h_{22}\right)$ : Similar to the previous bounds, we start from Lemma 7 and write

$$
\begin{aligned}
R_{1}+R_{2} \leq & I\left(y_{1}^{\ell}, y_{2}^{\ell} ; x_{1}^{\ell}, x_{2}^{\ell}\right)+\ell \varepsilon_{\ell} \\
\leq & I\left(y_{1}^{\prime \ell}, y_{2}^{\ell} ; x_{1}^{\ell}, x_{2}^{\ell}\right)+\ell \varepsilon_{\ell} \\
\leq & I\left(y_{1}^{\ell \ell}, y_{2}^{\ell} ; x_{1}^{\ell}, x_{2}^{\ell}, x_{2}^{\prime \ell}\right)+\ell \varepsilon_{\ell} \\
= & I\left(y_{1}^{\prime \ell} ; x_{1}^{\ell}, x_{2}^{\ell}\right)+I\left(y_{1}^{\prime \ell} ; x_{2}^{\prime \ell} \mid x_{1}^{\ell}, x_{2}^{\ell}\right)+I\left(y_{2}^{\ell} ; x_{2}^{\prime \ell} \mid y_{1}^{\prime \ell}\right) \\
& +I\left(y_{2}^{\ell} ; x_{1}^{\ell}, x_{2}^{\ell} \mid y_{1}^{\prime \ell}, x_{2}^{\prime \ell}\right)+\ell \varepsilon_{\ell} \\
= & I\left(y_{1}^{\ell \ell} ; x_{1}^{\ell}, x_{2}^{\ell}\right)+I\left(y_{2}^{\ell} ; x_{2}^{\prime \ell} \mid y_{1}^{\prime \ell}\right)+\ell \varepsilon_{\ell} \\
\leq & \frac{\ell}{2} \log \left(1+g_{11}+g_{12}\right)+I\left(y_{2}^{\ell} ; x_{2}^{\prime \ell} \mid y_{1}^{\prime \ell}\right)+\ell \varepsilon_{\ell} . \text { (A.24) }
\end{aligned}
$$

Note that in (A.22) we used the data processing inequality. An argument similar to that is used in the proof of (GZS-4) shows that the second and fourth terms in (A.23) are zero. Now, we have

$$
\begin{aligned}
I\left(y_{2}^{\ell} ; x_{2}^{\prime \ell} \mid y_{1}^{\prime \ell}\right) & =h\left(y_{2}^{\ell} \mid y_{1}^{\prime \ell}\right)-h\left(y_{2}^{\ell} \mid x_{2}^{\prime \ell}, y_{1}^{\prime \ell}\right) \\
& \leq h\left(y_{2}^{\ell} \mid x_{1}^{\prime \ell}\right)-h\left(y_{2}^{\ell} \mid x_{1}^{\prime \ell}, x_{2}^{\prime \ell}, y_{1}^{\prime \ell}\right) \\
& =h\left(\sqrt{h_{22}} x_{2}^{\prime \ell}+z_{2}^{\ell} \mid x_{1}^{\prime \ell}\right)-h\left(z_{2}^{\ell} \mid x_{1}^{\prime \ell}, x_{2}^{\prime \ell}, y_{1}^{\prime \ell}\right) \\
& \leq h\left(\sqrt{h_{22}} x_{2}^{\prime \ell}+z_{2}^{\ell}\right)-h\left(z_{2}^{\ell}\right) \\
& \leq \frac{\ell}{2} \log \left(1+h_{22}\right) .
\end{aligned}
$$

Finally, we obtain the desired bound by replacing (A.25) in (A.24). It is worth mentioning that this bound is the same as the cut-set bound for the cut $\Omega_{s}=\left\{S_{1}, S_{2}, B\right\}$ and $\Omega_{d}=\left\{A, D_{1}, D_{2}\right\}$.

- (GZS-7) $R_{1}<\frac{1}{2} \log \left(1+h_{11}\right)$ : Using Lemma 7 and the data processing inequality, we can write

$\ell R_{1} \leq I\left(x_{1}^{\prime \ell} ; y_{1}^{\ell}\right)+\ell \varepsilon_{\ell} \leq I\left(x_{1}^{\prime \ell} ; y_{1}^{\ell}\right)+\ell \varepsilon_{\ell} \leq \frac{\ell}{2} \log \left(1+h_{11}\right)+\ell \varepsilon_{\ell}$.

- (GZS-8) $R_{2}<\frac{1}{2} \log \left(1+h_{21}+h_{22}+2 \sqrt{h_{21} h_{22}}\right)$ : Starting from Lemma 7 and applying the data processing inequality for the Markov chain $x_{2}^{\ell} \leftrightarrow\left(y_{1}^{\prime \ell}, y_{2}^{\prime \ell}\right) \leftrightarrow\left(x_{1}^{\prime \ell}, x_{2}^{\prime \ell}\right) \leftrightarrow y_{2}^{\ell}$, we have

$$
\begin{aligned}
\ell R_{2} & \leq I\left(x_{2}^{\ell} ; y_{2}^{\ell}\right)+\ell \varepsilon_{\ell} \leq I\left(x_{1}^{\prime \ell}, x_{2}^{\prime \ell} ; y_{2}^{\ell}\right)+\ell \varepsilon_{\ell} \\
& \leq \frac{\ell}{2} \log \left(1+h_{21}+h_{22}+2 \sqrt{h_{21} h_{22}}\right)+\ell \varepsilon_{\ell} .
\end{aligned}
$$

Note that $x_{1}^{\prime \ell}$ and $x_{2}^{\prime \ell}$ are not independent. However, their variance is upper bounded by $\left(\sqrt{h_{21}}+\sqrt{h_{22}}\right)^{2}$.

- (GZS-9) $R_{2}<\frac{1}{2} \log \left(1+g_{22}\right)+\frac{1}{2} \log \left(1+h_{21}\right)$ : Consider the cut which partitions the network into $\Omega_{s}=\left\{S_{1}, S_{2}, A, D_{1}\right\}$ and $\Omega_{d}=\left\{B, D_{2}\right\}$. We have

$$
\begin{aligned}
\ell R_{2} \leq & I\left(x_{2}^{\ell} ; y_{2}^{\ell}\right)+\ell \varepsilon_{\ell} \\
\leq & I\left(x_{1}^{\prime \ell}, x_{2}^{\ell} ; y_{2}^{\prime \ell}, y_{2}^{\ell}\right)+\ell \varepsilon_{\ell} \\
= & I\left(x_{2}^{\ell} ; y_{2}^{\prime \ell}\right)+I\left(x_{1}^{\prime \ell} ; y_{2}^{\prime \ell} \mid x_{2}^{\ell}\right)+I\left(x_{1}^{\prime \ell} ; y_{2}^{\ell} \mid y_{2}^{\prime \ell}\right) \\
& +I\left(x_{2}^{\ell} ; y_{2}^{\ell} \mid y_{2}^{\prime \ell}, x_{1}^{\prime \ell}\right)+\ell \varepsilon_{\ell} \\
= & I\left(x_{2}^{\ell} ; y_{2}^{\prime \ell}\right)+I\left(x_{1}^{\prime \ell} ; y_{2}^{\ell} \mid y_{2}^{\prime \ell}\right)+\ell \varepsilon_{\ell} \\
= & I\left(x_{2}^{\ell} ; y_{2}^{\prime \ell}\right)+I\left(x_{1}^{\prime \ell} ; y_{2}^{\ell} \mid y_{2}^{\prime \ell}, x_{2}^{\prime \ell}\right)+\ell \varepsilon_{\ell} \\
\leq & \frac{\ell}{2} \log \left(1+g_{22}\right)+\frac{\ell}{2} \log \left(1+h_{21}\right)+\ell \varepsilon_{\ell} .
\end{aligned}
$$

We again used an argument similar to that is used in proof of (GZS-4) to show that the second and fourth terms in (A.28) are zero.

This completes the proof of the outer bound in Theorem 2 .

B) Achievability Proof: In this section, we provide an encoding scheme for the Gaussian ZS network, and show that the rate region that can be achieved using this scheme is only a constant bit gap away from the outer bound.

Large Channel Gains: In this part, we assume that all channel gains are at least 1 , i.e., $g_{i j} \geq 1$, and $h_{i j} \geq 1$. Note that if any of the gains is small, then either one of the rates is small (of the order of our constant bit gap), or the cross links are negligible. We will discuss these cases later.

The encoding scheme proposed for the Gaussian ZS network consists of two separate parts. We first split the message of the second source nodes as $W_{1}=U_{1}^{(1)}$ and $W_{2}=\left(U_{2}^{(1)}, U_{2}^{(2)}, U_{2}^{(3)}\right)$, where $U_{2}^{(1)}$ can be decoded at both relay nodes $A$ and $B$, and $U_{2}^{(2)}$ and $U_{2}^{(3)}$ can be decoded only at $A$ and $B$, respectively (see Fig. 10). Denoting the rate of message $W_{i}^{(j)}$ by $\Upsilon_{i, j}$, the following rate constraints are imposed by this message splitting

$$
\begin{aligned}
& R_{1}=\Upsilon_{1,1} \\
& R_{2}=\Upsilon_{2,1}+\Upsilon_{2,2}+\Upsilon_{2,3} .
\end{aligned}
$$

An achievable rate region for this message splitting is given in Lemma 1.

In the second layer of the network (see Fig. 11), relay node $A$ further splits its messages as follows: $W_{1}=U_{1}^{(1)}=\left(V_{1}^{(1)}, V_{1}^{(2)}\right), U_{2}^{(1)}=\left(V_{2}^{(1)}, V_{2}^{(2)}\right)$, and $U_{2}^{(2)}=\left(V_{2}^{(3)}, V_{2}^{(4)}\right)$. A similar message splitting is also performed at node $B$ to obtain $U_{2}^{(1)}=\left(V_{2}^{(1)}, V_{2}^{(2)}\right)$ and $U_{2}^{(3)}=V_{2}^{(5)}$. This message splitting imposes the following rate equations:

$$
\begin{aligned}
& \Upsilon_{1,1}=\Theta_{1,1}+\Theta_{1,2} \\
& \Upsilon_{2,1}=\Theta_{2,1}+\Theta_{2,2} \\
& \Upsilon_{2,2}=\Theta_{2,3}+\Theta_{2,4} \\
& \Upsilon_{2,3}=\Theta_{2,5}
\end{aligned}
$$

where $\Theta_{i, j}$ denotes the rate of the message $V_{i}^{(j)}$. Next, the relay nodes have to convey the messages to the destination nodes such that $D_{1}$ can decode $V_{1}^{(1)}, V_{1}^{(2)}, V_{2}^{(1)}$ and $V_{2}^{(3)}$, and $D_{2}$ be able to decode $V_{1}^{(1)}, V_{2}^{(1)}, V_{2}^{(2)}, V_{2}^{(3)}, V_{2}^{(4)}$ and $V_{2}^{(5)}$. An achievable rate region for this transmission scenario is given in Lemma 2.

Putting the rate constraints in Lemma 1 and Lemma 2 together with the equations in (A.30)-(A.31) and (A.32)-(A.35), we obtain the following achievable rate region for the Gaussian ZS network:

$$
\begin{aligned}
& \mathcal{R}_{\text {ach }}^{\text {GZS }}=\left\{\left(R_{1}, R_{2}\right): \exists \Upsilon_{1,1}, \Upsilon_{2,1}, \Upsilon_{2,2}, \Upsilon_{2,3}\right. \\
& \Theta_{1,1}, \Theta_{1,2}, \Theta_{2,1}, \Theta_{2,2}, \Theta_{2,3}, \Theta_{2,4}, \Theta_{2,5} \geq 0 \\
& R_{1}=\Upsilon_{1,1} \\
& R_{2}=\Upsilon_{2,1}+\Upsilon_{2,2}+\Upsilon_{2,3} \\
& \Upsilon_{1,1}=\Theta_{1,1}+\Theta_{1,2} \\
& \Upsilon_{2,1}=\Theta_{2,1}+\Theta_{2,2} \\
& \Upsilon_{2,2}=\Theta_{2,3}+\Theta_{2,4}
\end{aligned}
$$




$$
\begin{aligned}
\Upsilon_{2,3} & =\Theta_{2,5} \\
\Upsilon_{1,1} & \leq\left(\frac{1}{2} \log \left(1+g_{11}\right)-\frac{1}{2}\right)^{+} \\
\Upsilon_{2,2} & \leq\left(\frac{1}{2} \log \left(1+\frac{g_{12}}{g_{22}}\right)-\frac{1}{2}\right)^{+} \\
\Upsilon_{2,1}+\Upsilon_{2,2} & \leq\left(\frac{1}{2} \log \left(1+g_{12}\right)-\frac{1}{2}\right)^{+} \\
\Upsilon_{1,1}+\Upsilon_{2,1}+\Upsilon_{2,2} & \leq\left(\frac{1}{2} \log \left(1+g_{11}+g_{12}\right)-\frac{1}{2}\right)^{+} \\
\Upsilon_{2,3} & \leq\left(\frac{1}{2} \log \left(1+\frac{g_{22}}{g_{12}}\right)-\frac{1}{2}\right)^{+} \\
\Upsilon_{2,1}+\Upsilon_{2,3} & \leq\left(\frac{1}{2} \log \left(1+g_{22}\right)-\frac{1}{2}\right)^{+} \\
\Theta_{1,1}+\Theta_{1,2}+\Theta_{2,1}+\Theta_{2,3} & \leq\left(\frac{1}{2} \log \left(1+h_{11}\right)-\frac{1}{2}\right)^{+} \\
\Theta_{1,2} & \leq\left(\frac{1}{2} \log \left(1+\frac{h_{11}}{h_{12}}\right)-\frac{1}{2}\right)^{+} \\
\Theta_{2,4} & \leq\left(\frac{1}{2} \log \left(1+\frac{h_{21}}{h_{11}}\right)-\frac{1}{2}\right)^{+} \\
\Theta_{1,1}+\Theta_{2,3}+\Theta_{2,4} & \leq\left(\frac{1}{2} \log \left(1+h_{21}\right)-\frac{1}{2}\right)^{+} \\
\Theta_{2,5} & \leq\left(\frac{1}{2} \log \left(1+h_{22}\right)-\frac{1}{2}\right)^{+} \\
\Theta_{1,1}+\Theta_{2,1}+\Theta_{2,2} & +\Theta_{2,3}+\Theta_{2,4}+\Theta_{2,5} \\
& \left.\leq\left(\frac{1}{2} \log \left(1+h_{21}+h_{22}\right)-\frac{1}{2}\right)^{+}\right\} .
\end{aligned}
$$

We apply the Fourier-Motzkin elimination on this region, to project it on the coordinated $R_{1}$ and $R_{2}$, and obtain the following rate region. After some simplifications, we get

$$
\begin{aligned}
\mathcal{R}_{\mathrm{ach}}^{\mathrm{GZS}} & =\left\{\left(R_{1}, R_{2}\right): R_{1} \leq\left(\frac{1}{2} \log \left(g_{11}\right)-\frac{1}{2}\right)^{+}\right. \\
R_{2} & \leq\left(\frac{1}{2} \log \left(g_{12}+g_{22}\right)-\frac{1}{2}\right)^{+} \\
R_{1}+R_{2} & \leq\left(\frac{1}{2} \log \left(g_{11}+g_{12}\right)+\frac{1}{2} \log \left(\frac{g_{22}}{g_{12}}\right)-\frac{1}{2}\right)^{+} \\
R_{2} & \leq\left(\frac{1}{2} \log \left(g_{12}\right)+\frac{1}{2} \log \left(h_{22}\right)-\frac{1}{2}\right)^{+} \\
R_{1}+R_{2} & \leq\left(\frac{1}{2} \log \left(g_{22}\right)+\frac{1}{2} \log \left(h_{11}+h_{21}\right)-\frac{1}{2}\right)^{+} \\
R_{1}+R_{2} & \leq\left(\frac{1}{2} \log \left(g_{11}+g_{12}\right)+\frac{1}{2} \log \left(h_{22}\right)-\frac{1}{2}\right)^{+} \\
R_{1} & \leq\left(\frac{1}{2} \log \left(h_{11}\right)-\frac{1}{2}\right)^{+} \\
R_{2} & \leq\left(\frac{1}{2} \log \left(h_{21}+h_{22}\right)-\frac{1}{2}\right)^{+} \\
R_{2} & \leq\left(\frac{1}{2} \log \left(g_{22}\right)+\frac{1}{2} \log \left(h_{21}\right)-\frac{1}{2}\right)^{+} \\
R_{1}+R_{2} & \left.\leq\left(\frac{1}{2} \log \left(h_{21}+h_{22}\right)+\frac{1}{2} \log \left(\frac{h_{11}}{h_{21}}\right)-\frac{1}{2}\right)^{+}\right\} .
\end{aligned}
$$

Note that this rate region is characterized by a set of constraints which are similar to the inequalities in the definition of $\mathcal{R}^{\mathrm{GZS}}$, except for the additive constants, and the fact that $\log (1+x)$ is replaced by $\log (x)$. Note that since $x \geq 1$, we have

$$
\frac{1}{2} \log (1+x)-\frac{1}{2} \log (x) \leq \frac{1}{2} .
$$

Hence, the difference between the RHS's of two sets of inequalities do not exceed 1 for $R_{1}$, and $3 / 2$ for $R_{2}$ and $R_{1}+R_{2}$. Therefore, for any rate pair $\left(R_{1}, R_{2}\right) \in \mathcal{R}^{\mathrm{GZS}}$, we have $\left(R_{1}-1, R_{2}-\right.$ 1.5) $\in \mathcal{R}_{\text {ach }}^{\mathrm{GZS}}$. This completes the proof.

Small Channel Gains: We will show in this part that if any of the channel gains is small, then the outer bound in Theorem 2 is still within a constant bit gap of an achievable rate region. This argument is based on the analysis of a modified version of the network, in which all the links with gain smaller than 1 are removed. One can show that the capacity region of this modified network is within a constant gap from that of the original one. On the other hand, we can argue that the gap between the achievable rate pairs of the modified network and the outer bound in Theorem 2 is bounded by a constant. Therefore, we can conclude that if $\left(R_{1}, R_{2}\right) \in \mathcal{R}^{\mathrm{GZS}}$ then $\left(R_{1}-\delta_{1}, R_{2}-\delta_{2}\right)$ is achievable for the original network, where $\delta_{1}=1$ and $\delta_{2}=1.5$.

The main intuition behind this argument is the fact that since all the nodes are assumed to have power constraint equal to 1 , the flow of information through a link with gain not exceeding 1 is upper bounded by $\frac{1}{2} \log (1+$ SNR $) \leq \frac{1}{2} \log (1+1)=\frac{1}{2}$ bit. Therefore, by removing such links from the network, the achievable rates change by at most $\frac{1}{2}$ bit. On the other hand, the incoming signals over small channel gains may act as an interference on the original network, which cause a total noise power not exceeding 1 . Therefore, by doubling the noise variances of the original network, we guarantee that capacity region of the modified network is always smaller than that of the original one.

The advantage of analyzing the modified network instead of the original one is that some of the links are removed in the modified network, which convert it to simpler network to analyze.

A precise analysis of the modified networks requires considering several cases separately. However, similar techniques and ideas will be used for all cases. In the following we present one illustrating example, and skip the details for the other cases.

Example 5: Consider the Gaussian ZS network in Fig. 3(a), and assume that $g_{12}=0$. Therefore, the first layer of the network would be two parallel links as shown in Fig. 16, where $\mathbb{E}\left[\tilde{z}_{1}^{\prime 2}\right]=2$. Moreover, the rate region in (GZS-1)-(GZS-10) will be reduced to

$$
\begin{aligned}
R_{1} & \leq \frac{1}{2} \log \left(1+g_{11}\right) \\
R_{2} & \leq \frac{1}{2} \log \left(1+g_{12}\right) \\
R_{1} & \leq \frac{1}{2} \log \left(1+h_{11}\right) \\
R_{2} & \leq \frac{1}{2} \log \left(1+h_{22}\right) \\
R_{1}+R_{2} & \leq \frac{1}{2} \log \left(1+h_{21}+h_{22}+2 \sqrt{h_{21} h_{22}}\right) \\
& +\frac{1}{2} \log \left(1+\frac{h_{11}}{h_{21}}\right) .
\end{aligned}
$$




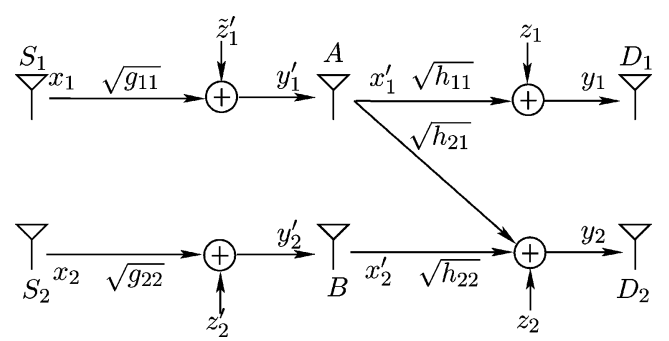

Fig. 16. Modified ZS network obtained assuming $g_{12}=0$.

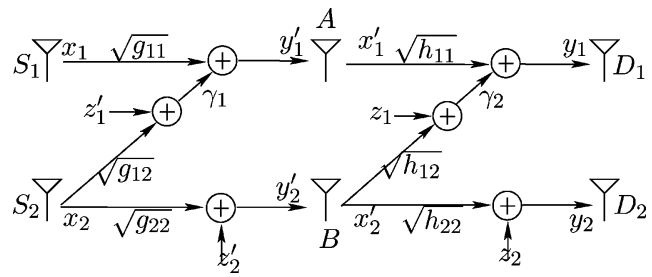

Fig. 17. Gaussian ZZ network.

The encoding strategy for this network is fairly simple. Let $\left(R_{1}, R_{2}\right)$ be a rate pair satisfying (A.38)-(A.42). The goal is to show that $\left(R_{1}-1, R_{2}-1\right)$ is achievable. Since $\left(R_{1}-1, R_{2}-1\right)$ satisfies (A.38) and (A.39), transmission over the first layer of the network from the source nodes to the relays is simply done using random Gaussian codes.

The second layer of the network is a Gaussian S network. Once the relays decode the messages received from the first layer of the network, they encode them using an encoding strategy similar to that of the $Z$ network in Example 4 in Section IV. Note that the sum-rate bounds in (A.42) and the outer bound of the $\mathrm{S}$ network are slightly different. However, their difference is upper bounded by

$$
\begin{aligned}
\frac{1}{2} \log & \left(1+h_{21}+h_{22}+2 \sqrt{h_{21} h_{22}}\right)-\frac{1}{2} \log \left(1+h_{21}+h_{22}\right) \\
= & \frac{1}{2} \log \left(1+\frac{2 \sqrt{h_{21} h_{22}}}{1+h_{21}+h_{22}}\right) \\
< & \frac{1}{2} \log (1+1)=\frac{1}{2} .
\end{aligned}
$$

Therefore, the loss caused by this difference is at most $\frac{1}{2}$ bit, and $\left(R_{1}-1, R_{2}-1\right)$ would be achievable. On the other hand, as we argued before, the capacity of the modified network is an inner bound for the original one, and hence, $\left(R_{1}-1, R_{2}-1\right)$ is achievable for the ZS network as well.

\section{APPENDIX B \\ GAUSSIAN ZZ NETWORK}

A) Outer Bound: In the following we present the proof for each of the inequalities in (GZZ-1)-(GZZ-6), separately. We again present the Gaussian ZZ network in Fig. 17, to clarify the notation used in the proof. In particular, we use two variables, which are the noisy signals received at $A$ and $D_{1}$ through the cross links assuming the direct links were absent, namely

$$
\begin{aligned}
& \gamma_{1}=\sqrt{g_{12}} x_{2}+z_{1}^{\prime} \\
& \gamma_{2}=\sqrt{h_{12}} x_{2}^{\prime}+z_{1} .
\end{aligned}
$$

Note that $y_{1}^{\prime}=\sqrt{g_{11}} x_{1}+\gamma_{1}$ and $y_{1}=\sqrt{h_{11}} x_{1}^{\prime}+\gamma_{2}$.
Suppose that the rate pair $\left(R_{1}, R_{2}\right)$ is achieved with a small decoding error probability $\varepsilon_{\ell}$ using a code of length $\ell$. The following chains of inequalities provide upper bounds on the individual rates as well as the sum-rate. We again use Lemma 7 , which essentially captures the decodability requirements of the network.

The individual rate bounds in (GZZ-1)-(GZZ-4) have the same structure as the cut-set bound, although we derive them through a slightly different argument. However, the two sum-rate bounds in (GZZ-5) and (GZZ-6) are conceptually different than the cut-set bounds. These two bounds which are tighter than cut-set bounds are derived through a genie-aided argument; that is, we assume that the signal sent over the cross link of one layer is given by a genie to the receiver of the other layer (relay node $A$ in layer 1 and destination node $D_{1}$ in layer 2). Therefore, we present the proofs of (GZZ-5) and (GZZ-6) first. The more standard cut-set type bounds are provided later for completeness.

\section{1) Proof of the Genie-Aided Bounds}

- (GZZ-5)

$$
\begin{aligned}
R_{1}+R_{2} \leq \frac{1}{2} \log (1+ & \left.g_{11}+g_{12}\right) \\
& +\frac{1}{2} \log \left(1+\frac{g_{22}}{g_{12}}\right)+\frac{1}{2} \log \left(1+h_{12}\right):
\end{aligned}
$$

We start with the sum-rate inequality in Lemma 7 , and write

$$
\begin{aligned}
\ell & \left(R_{1}+R_{2}\right) \\
\leq & I\left(y_{1}^{\ell}, y_{2}^{\ell} ; x_{1}^{\ell}, x_{2}^{\ell}\right)+\ell \varepsilon_{\ell} \\
\leq & I\left(y_{1}^{\prime \ell}, y_{2}^{\prime \ell} ; x_{1}^{\ell}, x_{2}^{\ell}\right)+\ell \varepsilon_{\ell} \\
\leq & I\left(y_{1}^{\prime \ell}, y_{2}^{\prime \ell}, \gamma_{2}^{\ell} ; x_{1}^{\ell}, x_{2}^{\ell}\right)+\ell \varepsilon_{\ell} \\
= & I\left(y_{1}^{\prime \ell}, \gamma_{2}^{\ell} ; x_{1}^{\ell}, x_{2}^{\ell}\right)+I\left(y_{2}^{\prime \ell} ; x_{1}^{\ell}, x_{2}^{\ell} \mid y_{1}^{\prime \ell}, \gamma_{2}^{\ell}\right)+\ell \varepsilon_{\ell} \\
\leq & I\left(y_{1}^{\prime \ell}, \gamma_{2}^{\ell} ; x_{1}^{\ell}, x_{2}^{\ell}\right)+I\left(y_{2}^{\prime \ell}, \gamma_{1}^{\ell} ; x_{1}^{\ell}, x_{2}^{\ell} \mid y_{1}^{\prime \ell}, \gamma_{2}^{\ell}\right)+\ell \varepsilon_{\ell} \\
= & I\left(y_{1}^{\prime \ell}, \gamma_{2}^{\ell} ; x_{1}^{\ell}, x_{2}^{\ell}\right)+I\left(\gamma_{1}^{\ell} ; x_{1}^{\ell}, x_{2}^{\ell} \mid y_{1}^{\prime \ell}, \gamma_{2}^{\ell}\right) \\
& +I\left(y_{2}^{\prime \ell} ; x_{1}^{\ell}, x_{2}^{\ell} \mid y_{1}^{\prime \ell}, \gamma_{1}^{\ell}, \gamma_{2}^{\ell}\right)+\ell \varepsilon_{\ell} \\
= & I\left(y_{1}^{\prime \ell}, \gamma_{2}^{\ell} ; x_{1}^{\ell}, x_{2}^{\ell}\right)+I\left(\gamma_{1}^{\ell} ; x_{1}^{\ell}, x_{2}^{\ell} \mid y_{1}^{\prime \ell}, \gamma_{2}^{\ell}\right) \\
& +I\left(y_{2}^{\prime \ell} ; x_{2}^{\ell} \mid y_{1}^{\prime \ell}, \gamma_{1}^{\ell}, \gamma_{2}^{\ell}\right)+I\left(y_{2}^{\prime \ell} ; x_{1}^{\ell} \mid x_{2}^{\ell}, y_{1}^{\prime \ell}, \gamma_{1}^{\ell}, \gamma_{2}^{\ell}\right)+\ell \varepsilon_{\ell} .
\end{aligned}
$$

Each of the terms in (B.1) can be bounded as follows. In order to bound the first term, we can simply write

$$
\begin{aligned}
I\left(y_{1}^{\prime \ell}, \gamma_{2}^{\ell} ; x_{1}^{\ell}, x_{2}^{\ell}\right) & =I\left(\gamma_{2}^{\ell} ; x_{1}^{\ell}, x_{2}^{\ell}\right)+I\left(y_{1}^{\prime \ell} ; x_{1}^{\ell}, x_{2}^{\ell} \mid \gamma_{2}^{\ell}\right) \\
& =I\left(\gamma_{2}^{\ell} ; x_{1}^{\ell}, x_{2}^{\ell}\right)+h\left(y_{1}^{\prime \ell} \mid \gamma_{2}^{\ell}\right)-h\left(y_{1}^{\prime \ell} \mid x_{1}^{\ell}, x_{2}^{\ell}, \gamma_{2}^{\ell}\right) \\
& \leq I\left(\gamma_{2}^{\ell} ; x_{1}^{\ell}, x_{2}^{\ell}\right)+h\left(y_{1}^{\prime \ell}\right)-h\left(y_{1}^{\prime \ell} \mid x_{1}^{\ell}, x_{2}^{\ell}\right) \quad(\mathrm{B} .2) \\
& =I\left(\gamma_{2}^{\ell} ; x_{1}^{\ell}, x_{2}^{\ell}\right)+I\left(y_{1}^{\prime \ell} ; x_{1}^{\ell}, x_{2}^{\ell}\right) \\
& \leq I\left(\gamma_{2}^{\ell} ; x_{2}^{\prime \ell}\right)+I\left(y_{1}^{\prime \ell} ; x_{1}^{\ell}, x_{2}^{\ell}\right) \\
& =\frac{\ell}{2} \log \left(1+h_{12}\right)+\frac{\ell}{2} \log \left(1+g_{11}+g_{12}\right)
\end{aligned}
$$

where in (B.2) we have used the fact that conditioning decreases the entropy, and the Markov chain $\gamma_{2}^{\ell} \leftrightarrow x_{2}^{\ell \ell} \leftrightarrow$ 
$y_{2}^{\prime \ell} \leftrightarrow\left(x_{1}^{\ell}, x_{2}^{\ell}\right) \leftrightarrow y_{1}^{\prime \ell}$. Also (B.3) follows from the same Markov chain.

For the second term, we can write

$$
\begin{aligned}
I\left(\gamma_{1}^{\ell} ; x_{1}^{\ell}, x_{2}^{\ell} \mid y_{1}^{\prime \ell}, \gamma_{2}^{\ell}\right) & =I\left(y_{1}^{\prime \ell}-\gamma_{1}^{\ell} ; x_{1}^{\ell}, x_{2}^{\ell} \mid y_{1}^{\prime \ell}, \gamma_{2}^{\ell}\right) \\
& =I\left(\sqrt{g_{11}} x_{1}^{\ell} ; x_{1}^{\ell}, x_{2}^{\ell} \mid y_{1}^{\prime \ell}, \gamma_{2}^{\ell}\right) \\
& \leq I\left(W_{1} ; x_{1}^{\ell}, x_{2}^{\ell} \mid y_{1}^{\prime \ell}, \gamma_{2}^{\ell}\right) \\
& \leq H\left(W_{1} \mid y_{1}^{\prime \ell}, \gamma_{2}^{\ell}\right) \\
& \leq H\left(W_{1} \mid y_{1}^{\ell}\right) \leq \ell \varepsilon_{\ell}
\end{aligned}
$$

where the last inequality holds since $y_{1}^{\ell}=\sqrt{h_{11}} x_{1}^{\prime \ell}+\gamma_{2}^{\ell}=$ $f_{1}\left(y_{1}^{\prime \ell}\right)+\gamma_{2}^{\ell}=f_{2}\left(y_{1}^{\prime \ell}, \gamma_{2}^{\ell}\right)$.

In order to bound the third term in (B.1) we can write

$I\left(y_{2}^{\prime \ell} ; x_{2}^{\ell} \mid y_{1}^{\prime \ell}, \gamma_{1}^{\ell}, \gamma_{2}^{\ell}\right)$

$=h\left(x_{2}^{\ell} \mid y_{1}^{\prime \ell}, \gamma_{1}^{\ell}, \gamma_{2}^{\ell}\right)-h\left(x_{2}^{\ell} \mid y_{1}^{\prime \ell}, y_{2}^{\prime \ell}, \gamma_{1}^{\ell}, \gamma_{2}^{\ell}\right)$

$\leq h\left(x_{2}^{\ell} \mid \gamma_{1}^{\ell}\right)-h\left(x_{2}^{\ell} \mid y_{1}^{\prime \ell}, y_{2}^{\ell \ell}, \gamma_{1}^{\ell}, \gamma_{2}^{\ell}\right)$

$=h\left(x_{2}^{\ell} \mid \gamma_{1}^{\ell}\right)-\left[h\left(x_{2}^{\ell}, t_{1}^{\ell} \mid y_{1}^{\prime \ell}, y_{2}^{\prime \ell}, \gamma_{2}^{\ell}\right)-h\left(t_{1}^{\ell} \mid y_{1}^{\prime \ell}, y_{2}^{\prime \ell}, \gamma_{2}^{\ell}\right)\right]$

$=h\left(x_{2}^{\ell} \mid \gamma_{1}^{\ell}\right)-\left[h\left(x_{2}^{\ell}, t_{1}^{\ell} \mid y_{1}^{\prime \ell}, y_{2}^{\prime \ell}\right)-h\left(t_{1}^{\ell} \mid y_{1}^{\prime \ell}, y_{2}^{\prime \ell}\right)\right]$

$=h\left(x_{2}^{\ell} \mid \gamma_{1}^{\ell}\right)-h\left(x_{2}^{\ell} \mid y_{1}^{\prime \ell}, y_{2}^{\prime \ell}, \gamma_{1}^{\ell}\right)$

$=h\left(x_{2}^{\ell} \mid \gamma_{1}^{\ell}\right)-h\left(x_{2}^{\ell} \mid x_{1}^{\ell}, y_{2}^{\prime \ell}, \gamma_{1}^{\ell}\right)$

$=h\left(x_{2}^{\ell} \mid \gamma_{1}^{\ell}\right)-\left[h\left(x_{2}^{\ell} \mid y_{2}^{\prime \ell}, \gamma_{1}^{\ell}\right)+h\left(x_{1}^{\ell} \mid x_{2}^{\ell}, y_{2}^{\prime \ell}, \gamma_{1}^{\ell}\right)-h\left(x_{1}^{\ell} \mid y_{2}^{\prime \ell}, \gamma_{1}^{\ell}\right)\right]$

$=h\left(x_{2}^{\ell} \mid \gamma_{1}^{\ell}\right)-h\left(x_{2}^{\ell} \mid y_{2}^{\prime \ell}, \gamma_{1}^{\ell}\right)$

$=I\left(y_{2}^{\ell} ; x_{2}^{\ell} \mid \gamma_{1}^{\ell}\right)$

$=h\left(y_{2}^{\ell \ell} \mid \gamma_{1}^{\ell}\right)-h\left(y_{2}^{\ell} \mid \gamma_{1}^{\ell}, x_{2}^{\ell}\right)$

$=h\left(y_{2}^{\prime \ell} \mid \gamma_{1}^{\ell}\right)-h\left(y_{2}^{\prime \ell} \mid x_{2}^{\ell}\right)$

$=h\left(y_{2}^{\prime \ell}-\sqrt{\frac{g_{22}}{g_{12}}} \gamma_{1}^{\ell} \mid \gamma_{1}^{\ell}\right)-h\left(y_{2}^{\ell \ell}-\sqrt{g_{22}} x_{2}^{\ell} \mid x_{2}^{\ell}\right)$

$=h\left(z_{2}^{\prime \ell}-\sqrt{\frac{g_{22}}{g_{12}}} z_{1}^{\prime \ell} \mid \gamma_{1}^{\ell}\right)-h\left(z_{2}^{\prime \ell} \mid x_{2}^{\ell}\right)$

$\leq h\left(z_{2}^{\prime \ell}-\sqrt{\frac{g_{22}}{g_{12}}} z_{1}^{\prime \ell}\right)-h\left(z_{2}^{\prime \ell}\right)$

$=\frac{\ell}{2} \log \left(1+\frac{g_{22}}{g_{12}}\right)$

where in (B.6) we have used the fact that conditioning reduces the differential entropy, and (B.7) holds due to the Markov chain $\left(x_{2}^{\ell}, t_{1}^{\ell}\right) \leftrightarrow\left(y_{1}^{\prime \ell}, y_{2}^{\prime \ell}\right) \leftrightarrow \gamma_{2}^{\ell}$. Then in (B.8) we replaced $\left(y_{1}^{\prime \ell}, \gamma_{1}^{\ell}\right)$ by $\left(x_{1}^{\ell}, \gamma_{1}^{\ell}\right)$ since there is an one-to-one map, $y_{1}^{\prime \ell}=\sqrt{g_{11}} x_{1}^{\ell}+t_{1}^{\ell}$, between these joint variables, and in (B.9) we used the fact that $x_{1}^{\ell}$ is independent of $\left(x_{2}^{\ell}, y_{2}^{\prime \ell}, t_{1}^{\ell}\right)$ to conclude $h\left(x_{1}^{\ell} \mid x_{2}^{\ell}, y_{2}^{\prime \ell}, \gamma_{1}^{\ell}\right)=$ $h\left(x_{1}^{\ell} \mid y_{2}^{\prime \ell}, \gamma_{1}^{\ell}\right)=h(x)$. Also (B.10) holds due to the Markov chain $y_{2}^{\prime \ell} \leftrightarrow x_{2}^{\ell} \leftrightarrow \gamma_{1}^{\ell}$. Finally, (B.11) is true due to removing conditioning and the fact that $z_{2}^{\prime}$ is independent of $x_{2}$.

Finally for the last term in (B.1) we have

$$
\begin{aligned}
I\left(y_{2}^{\prime \ell} ; x_{1}^{\ell} \mid x_{2}^{\ell}, y_{1}^{\prime \ell}, \gamma_{1}^{\ell}, \gamma_{2}^{\ell}\right) & \leq I\left(y_{2}^{\prime \ell} ; W_{1} \mid x_{2}^{\ell}, y_{1}^{\prime \ell}, \gamma_{1}^{\ell}, \gamma_{2}^{\ell}\right) \\
& \leq H\left(W_{1} \mid x_{2}^{\ell}, y_{1}^{\prime \ell}, \gamma_{1}^{\ell}, \gamma_{2}^{\ell}\right) \\
& \leq H\left(W_{1} \mid y_{1}^{\prime \ell}, \gamma_{2}^{\ell}\right) \\
& \leq H\left(W_{1} \mid y_{1}^{\ell}\right) \\
& \leq \ell \varepsilon_{\ell}
\end{aligned}
$$

where (B.13) is due to the fact that $y_{1}^{\ell}=\sqrt{h_{11}} x_{1}^{\prime \ell}+\gamma_{2}^{\ell}=$ $f_{1}\left(y_{1}^{\prime \ell}\right)+\gamma_{2}^{\ell}=f_{2}\left(y_{1}^{\prime \ell}, \gamma_{2}^{\ell}\right)$ is a function of $\left(y_{1}^{\prime \ell}, \gamma_{2}^{\ell}\right)$, and (B.14) is just the Fano's inequality.

Replacing (B.4), (B.5), (B.12), and (B.14) in (B.1), we get

$$
\begin{aligned}
R_{1}+R_{2} & \leq \frac{1}{2} \log \left(1+g_{11}+g_{12}\right) \\
+ & \frac{1}{2} \log \left(1+\frac{g_{22}}{g_{12}}\right)+\frac{1}{2} \log \left(1+h_{12}\right)+3 \ell \varepsilon_{\ell} .
\end{aligned}
$$

- (GZZ-6) Before proving this inequality, we present a lemma which will be used in this proof. We will present the proof of this lemma later in Appendix C.

Lemma 8: Let $X_{1}$ and $X_{2}$ be two (arbitrarily correlated) random variables with variance constraints $\mathbb{E}\left[X_{1}^{2}\right]=\sigma_{1}^{2}$ and $\mathbb{E}\left[X_{2}^{2}\right]=\sigma_{2}^{2}$, which form a Markov chain $X_{1} \leftrightarrow \Gamma \leftrightarrow X_{2}$ for some random variable $\Gamma$. Also assume that $Z$ is a zero-mean unit variance Gaussian random variable independent of $X_{1}, X_{2}$ and $\Gamma$. Then the conditional differential entropy of $Y=X_{1}+X_{2}+Z$ is upper bounded by

$$
h(Y \mid \Gamma) \leq \frac{1}{2} \log 2 \pi e\left(1+\sigma_{1}^{2}+\sigma_{2}^{2}\right) .
$$

Now, in order to prove (GZZ-5), we start with Lemma 7

$$
\begin{aligned}
\ell\left(R_{1}+R_{2}\right) \leq & I\left(y_{1}^{\ell}, y_{2}^{\ell} ; x_{1}^{\ell}, x_{2}^{\ell}\right)+\ell \varepsilon_{\ell} \\
\leq & I\left(y_{1}^{\ell}, y_{2}^{\ell}, \gamma_{1}^{\ell} ; x_{1}^{\ell}, x_{2}^{\ell}\right)+\ell \varepsilon_{\ell} \\
= & I\left(y_{1}^{\ell}, \gamma_{1}^{\ell} ; x_{1}^{\ell}, x_{2}^{\ell}\right)+I\left(y_{2}^{\ell} ; x_{1}^{\ell}, x_{2}^{\ell} \mid y_{1}^{\ell}, \gamma_{1}^{\ell}\right)+\ell \varepsilon_{\ell} \\
= & I\left(\gamma_{1}^{\ell} ; x_{1}^{\ell}, x_{2}^{\ell}\right)+I\left(y_{1}^{\ell} ; x_{1}^{\ell}, x_{2}^{\ell} \mid \gamma_{1}^{\ell}\right) \\
& +I\left(y_{2}^{\ell} ; x_{1}^{\ell}, x_{2}^{\ell} \mid y_{1}^{\ell}, \gamma_{1}^{\ell}\right)+\ell \varepsilon_{\ell} .
\end{aligned}
$$

Since $\gamma_{1}^{\ell}$ is independent of $x_{1}^{\ell}$, the first term can be simply bounded as

$$
\begin{aligned}
I\left(\gamma_{1}^{\ell} ; x_{1}^{\ell}, x_{2}^{\ell}\right) & =I\left(\gamma_{1}^{\ell} ; x_{2}^{\ell}\right)+I\left(t^{\ell} ; x_{1}^{\ell} \mid x_{2}^{\ell}\right) \\
& =I\left(\gamma_{1}^{\ell} ; x_{2}^{\ell}\right)+I\left(z_{1}^{\ell} ; x_{1}^{\ell} \mid x_{2}^{\ell}\right) \leq \frac{\ell}{2} \log \left(1+g_{12}\right)
\end{aligned}
$$

For the second term we can write

$$
\begin{aligned}
I\left(y_{1}^{\ell} ; x_{1}^{\ell}, x_{2}^{\ell} \mid \gamma_{1}^{\ell}\right) & =h\left(y_{1}^{\ell} \mid \gamma_{1}^{\ell}\right)-h\left(y_{1}^{\ell} \mid x_{1}^{\ell}, x_{2}^{\ell}, \gamma_{1}^{\ell}\right) \\
& \leq h\left(y_{1}^{\ell} \mid \gamma_{1}^{\ell}\right)-h\left(y_{1}^{\ell} \mid x_{1}^{\prime \ell}, x_{2}^{\prime \ell}\right) \\
& =h\left(y_{1}^{\ell} \mid \gamma_{1}^{\ell}\right)-h\left(z_{1}^{\ell} \mid x_{1}^{\prime \ell}, x_{2}^{\prime \ell}\right) \\
& =h\left(\sqrt{h_{11}} x_{1}^{\prime \ell}+\sqrt{h_{12}} x_{2}^{\prime \ell}+z_{1}^{\ell} \mid \gamma_{1}^{\ell}\right)-h\left(z_{1}^{\ell}\right) \\
& \leq \frac{\ell}{2} \log \left(2 \pi e\left(1+h_{11}+h_{12}\right)\right)-\frac{\ell}{2} \log 2 \pi e \\
& =\frac{\ell}{2} \log \left(1+h_{11}+h_{12}\right)
\end{aligned}
$$

where (B.19) follows from the Markov chain $y_{1}^{\ell} \leftrightarrow\left(x_{1}^{\prime \ell}, x_{2}^{\prime \ell}\right) \leftrightarrow$ $\left(x_{1}^{\ell}, x_{2}^{\ell}, \gamma_{1}^{\ell}\right)$. In (B.20) we have used Lemma 8 for $\gamma_{1}^{\ell}, x_{1}^{\prime \ell}$ and $x_{2}^{\prime \ell}$ which form a Markov chain, since

$$
\begin{aligned}
I\left(x_{1}^{\prime \ell} ; x_{2}^{\prime \ell} \mid \gamma_{1}^{\ell}\right) & \leq I\left(y_{1}^{\prime \ell} ; y_{2}^{\prime \ell} \mid \gamma_{1}^{\ell}\right)=I\left(\frac{y_{1}^{\prime \ell}-\gamma_{1}^{\ell}}{\sqrt{g_{11}}} ; y_{2}^{\prime \ell} \mid \gamma_{1}^{\ell}\right) \\
& =I\left(x_{1}^{\ell} ; y_{2}^{\prime \ell} \mid \gamma_{1}^{\ell}\right)=h\left(x_{1}^{\ell} \mid \gamma_{1}^{\ell}\right)-h\left(x_{1}^{\ell} \mid \gamma_{1}^{\ell}, y_{2}^{\prime \ell}\right)=0 .
\end{aligned}
$$


The third term in (B.17) can be further upper bounded by

$$
\begin{aligned}
& I\left(y_{2}^{\ell} ; x_{1}^{\ell}, x_{2}^{\ell} \mid y_{1}^{\ell}, \gamma_{1}^{\ell}\right) \\
& =h\left(y_{2}^{\ell} \mid y_{1}^{\ell}, \gamma_{1}^{\ell}\right)-h\left(y_{2}^{\ell} \mid x_{1}^{\ell}, x_{2}^{\ell}, y_{1}^{\ell}, \gamma_{1}^{\ell}\right) \\
& \leq h\left(y_{2}^{\ell} \mid y_{1}^{\ell}, \gamma_{1}^{\ell}\right)-h\left(y_{2}^{\ell} \mid x_{2}^{\prime \ell}\right) \\
& \leq h\left(y_{2}^{\ell} \mid y_{1}^{\ell}, \gamma_{1}^{\ell}\right)-h\left(y_{2}^{\ell} \mid x_{2}^{\prime \ell}, x_{1}^{\prime \ell}, y_{1}^{\ell}, \gamma_{1}^{\ell}\right) \\
& =I\left(y_{2}^{\ell} ; x_{1}^{\prime \ell}, x_{2}^{\prime \ell} \mid y_{1}^{\ell}, \gamma_{1}^{\ell}\right) \\
& \leq I\left(y_{2}^{\ell}, \gamma_{2}^{\ell} ; x_{1}^{\prime \ell}, x_{2}^{\prime \ell} \mid y_{1}^{\ell}, \gamma_{1}^{\ell}\right) \\
& =I\left(\gamma_{2}^{\ell} ; x_{1}^{\prime \ell}, x_{2}^{\prime \ell} \mid y_{1}^{\ell}, \gamma_{1}^{\ell}\right)+I\left(y_{2}^{\ell} ; x_{1}^{\prime \ell}, x_{2}^{\prime \ell} \mid y_{1}^{\ell}, \gamma_{1}^{\ell}, \gamma_{2}^{\ell}\right)
\end{aligned}
$$

where both (B.22) and (B.23) follow from the Markov chain $y_{2}^{\ell} \leftrightarrow x_{2}^{\prime \ell} \leftrightarrow\left(x_{1}^{\ell}, y_{1}^{\ell}, \gamma_{1}^{\ell}\right)$. Now, we have

$$
\begin{aligned}
I\left(\gamma_{2}^{\ell} ; x_{1}^{\prime \ell}, x_{2}^{\prime \ell} \mid y_{1}^{\ell}, \gamma_{1}^{\ell}\right) & =I\left(y_{1}^{\ell}-\gamma_{2}^{\ell} ; x_{1}^{\prime \ell}, x_{2}^{\prime \ell} \mid y_{1}^{\ell}, \gamma_{1}^{\ell}\right) \\
& =I\left(\sqrt{h_{11}} x_{1}^{\prime \ell} ; x_{1}^{\prime \ell}, x_{2}^{\prime \ell} \mid y_{1}^{\ell}, \gamma_{1}^{\ell}\right) \\
& \leq I\left(y_{1}^{\prime \ell} ; x_{1}^{\prime \ell}, x_{2}^{\prime \ell} \mid y_{1}^{\ell}, \gamma_{1}^{\ell}\right) \\
& \leq I\left(y_{1}^{\prime \ell}-t_{1}^{\ell} ; x_{1}^{\prime \ell}, x_{2}^{\prime \ell} \mid y_{1}^{\ell}, \gamma_{1}^{\ell}\right) \\
& =I\left(\sqrt{g_{11}} x_{1}^{\ell} ; x_{1}^{\prime \ell}, x_{2}^{\prime \ell} \mid y_{1}^{\ell}, \gamma_{1}^{\ell}\right) \\
& \leq I\left(W_{1} ; x_{1}^{\prime \ell}, x_{2}^{\prime \ell} \mid y_{1}^{\ell}, \gamma_{1}^{\ell}\right) \\
& \leq H\left(W_{1} \mid y_{1}^{\ell}\right) \leq \ell \varepsilon_{\ell}
\end{aligned}
$$

where (B.25) follows from the fact that $x_{1}^{\prime \ell}$ is a function of $y_{1}^{\prime \ell}$. Finally

$$
\begin{aligned}
I & \left(y_{2}^{\ell} ; x_{1}^{\prime \ell}, x_{2}^{\prime \ell} \mid y_{1}^{\ell}, \gamma_{1}^{\ell}, \gamma_{2}^{\ell}\right) \\
= & h\left(y_{2}^{\ell} \mid y_{1}^{\ell}, \gamma_{1}^{\ell}, \gamma_{2}^{\ell}\right)-h\left(y_{2}^{\ell} \mid y_{1}^{\ell}, \gamma_{1}^{\ell}, \gamma_{2}^{\ell}, x_{1}^{\prime \ell}, x_{2}^{\prime \ell}\right) \\
= & h\left(y_{2}^{\ell}-\sqrt{\frac{h_{22}}{h_{12}}} \gamma_{2}^{\ell} \mid y_{1}^{\ell}, \gamma_{1}^{\ell}, \gamma_{2}^{\ell}\right) \\
& -h\left(y_{2}^{\ell}-\sqrt{h_{22}} x_{2}^{\prime \ell} \mid y_{1}^{\ell}, \gamma_{1}^{\ell}, \gamma_{2}^{\ell}, x_{1}^{\prime \ell}, x_{2}^{\prime \ell}\right) \\
= & h\left(z_{2}^{\ell}-\sqrt{\frac{h_{22}}{h_{12}}} z_{1}^{\ell} \mid y_{1}^{\ell}, \gamma_{1}^{\ell}, \gamma_{2}^{\ell}\right)-h\left(z_{2}^{\ell} \mid y_{1}^{\ell}, \gamma_{1}^{\ell}, \gamma_{2}^{\ell}, x_{1}^{\prime \ell}, x_{2}^{\prime \ell}\right) \\
\leq & h\left(z_{2}^{\ell}-\sqrt{\frac{h_{22}}{h_{12}}} z_{1}^{\ell}\right)-h\left(z_{2}^{\ell}\right) \\
= & \frac{\ell}{2} \log \left(1+\frac{h_{22}}{h_{12}}\right) .
\end{aligned}
$$

Here, in (B.27) we have used the fact that conditioning decreases the differential entropy, and the fact that $z_{2}^{\ell}$ is independent of $\left(y_{1}^{\ell}, \gamma_{1}^{\ell}, \gamma_{2}^{\ell}, x_{1}^{\prime \ell}, x_{2}^{\prime \ell}\right)$. Replacing (B.18), (B.21), (B.26), and (B.28) in (B.17), we will obtain the desired inequality.

\section{2) Proofs of Cut-Set Type Bounds}

- (GZZ-1) $R_{1} \leq \frac{1}{2} \log \left(1+g_{11}\right)$ : The individual rate bound can be simply obtained from

$$
\begin{aligned}
\ell R_{1} & =I\left(x_{1}^{\ell} ; y_{1}^{\ell}\right)+\ell \varepsilon_{\ell} \\
& \leq I\left(x_{1}^{\ell} ; y_{1}^{\prime \ell}, y_{2}^{\prime \ell}\right)+\ell \varepsilon_{\ell} \\
& =I\left(x_{1}^{\ell} ; y_{1}^{\prime \ell} \mid y_{2}^{\prime \ell}\right)+I\left(x_{1}^{\ell} ; y_{2}^{\prime \ell}\right)+\ell \varepsilon_{\ell} \\
& =h\left(y_{1}^{\prime \ell} \mid y_{2}^{\prime \ell}\right)-h\left(y_{1}^{\prime \ell} \mid x_{1}^{\ell}, y_{2}^{\prime \ell}\right) \\
& \leq h\left(y_{1}^{\prime \ell} \mid x_{2}^{\ell}\right)-h\left(z_{1}^{\prime \ell} \mid x_{1}^{\ell}, y_{2}^{\prime \ell}\right) \\
& =h\left(\sqrt{g_{11}} x_{1}^{\ell}+z_{1}^{\prime \ell}\right)-h\left(z_{1}^{\prime \ell}\right)+\ell \varepsilon_{\ell} \\
& \leq \frac{\ell}{2} \log \left(1+g_{11}\right)+\ell \varepsilon_{\ell}
\end{aligned}
$$

where (B.29) follows from the data processing inequality for the Markov chain $x_{1}^{\ell} \leftrightarrow\left(y_{1}^{\prime \ell}, y_{2}^{\prime \ell}\right) \leftrightarrow y_{1}^{\ell}$, and (B.30) follows from the Markov chain $\left.y_{1}^{\prime \ell} \leftrightarrow x_{2}^{\ell} \leftrightarrow y_{2}^{\prime \ell}\right)$. Note that $\varepsilon_{\ell} \rightarrow 0$ as $\ell$ grows. It is worth mentioning that this bound is similar to the cut-set bound for the cut $\Omega_{s}=\left\{S_{1}\right\}$ and $\Omega_{d}=\left\{S_{2}, A, B, D_{1}, D_{2}\right\}$.

- $(\mathrm{GZZ}-2) R_{2} \leq \frac{1}{2} \log \left(1+g_{22}\right)$ :

For the second rate bound, we can start with Lemma 7 and write

$\ell R_{2} \leq I\left(x_{2}^{\ell} ; y_{2}^{\ell}\right)+\ell \varepsilon_{\ell} \leq I\left(x_{2}^{\ell} ; y_{2}^{\prime \ell}\right)+\ell \varepsilon_{\ell} \leq \frac{\ell}{2} \log \left(1+g_{22}\right)+\ell \varepsilon_{\ell}$

where we have used the data processing inequality and the Markov chain $x_{2}^{\ell} \leftrightarrow y_{2}^{\prime \ell} \leftrightarrow x_{2}^{\prime \ell} \leftrightarrow y_{2}^{\ell}$ in the second inequality. Note that this bound captures the maximum flow of information through the cut specified by $\Omega_{s}=\left\{S_{2}\right\}$ and $\Omega_{d}=\left\{S_{1}, A, B, D_{1}, D_{2}\right\}$.

- (GZZ-3) $R_{1} \leq \frac{1}{2} \log \left(1+h_{11}\right)$ : In order to prove this upper bound, we use the cut-set bound for the cut $\Omega_{s}=$ $\left\{S_{1}, S_{2}, A, B, D_{2}\right\}$ and $\Omega_{d}=\left\{D_{1}\right\}$

$$
\begin{aligned}
\ell R_{1} & \leq I\left(x_{1}^{\prime \ell} ; y_{1}^{\ell} \mid x_{2}^{\prime \ell}\right)+\ell \varepsilon_{\ell} \\
& =h\left(y_{1}^{\ell} \mid x_{2}^{\prime \ell}\right)-h\left(y_{1}^{\ell} \mid x_{1}^{\prime \ell}, x_{2}^{\prime \ell}\right)+\ell \varepsilon_{\ell} \\
& =h\left(\sqrt{h_{11}} x_{1}^{\prime \ell}+z_{1}^{\ell} \mid x_{2}^{\prime \ell}\right)-h\left(z_{1}^{\ell} \mid x_{1}^{\prime \ell}, x_{2}^{\prime \ell}\right)+\ell \varepsilon_{\ell} \\
& \leq h\left(\sqrt{h_{11}} x_{1}^{\prime \ell}+z_{1}^{\ell}\right)-h\left(z_{1}^{\ell}\right)+\ell \varepsilon_{\ell} \\
& \leq \frac{\ell}{2} \log \left(1+h_{11}\right)+\ell \varepsilon_{\ell} .
\end{aligned}
$$

- (GZZ-4) $R_{2} \leq \frac{1}{2} \log \left(1+h_{22}\right)$ : Starting from Lemma 7, we can write

$$
\ell R_{2} \leq I\left(x_{2}^{\prime \ell} ; y_{2}^{\ell}\right)+\ell \varepsilon_{\ell} \leq I\left(x_{2}^{\prime \ell} ; y_{2}^{\ell}\right)+\ell \varepsilon_{\ell} \leq \frac{1}{2} \log \left(1+h_{22}\right)+\ell \varepsilon_{\ell}
$$

where the second inequality follows from the data processing inequality for the Markov chain $x_{2}^{\ell} \leftrightarrow y_{2}^{\prime \ell} \leftrightarrow$ $x_{2}^{\prime \ell} \leftrightarrow y_{2}^{\ell}$.

This shows that the rate region in Theorem 4 is an outer bound for the achievable region region of the Gaussian ZZ network.

B) Achievability Proof: In this section we present an encoding/decoding scheme, and derive an achieve rate region for this strategy. We then show that the gap between the boundary of this achievable rate region and that of the outer bound presented in Theorem 4 is upper bounded by a constant.

Similar to the Gaussian ZS network, we only consider the large channel gain case, where we assume that all the channel gains are lower bounded by 1 . A similar argument to that we used for the ZS network shows that for small channel gain cases the network is reduced to a simple one and its gap analysis is fairly simple.

We essentially use the result of Lemma 3 as an achievable rate region for the Z-neutralization network. We use notation $\left(\lambda_{g}, \mu_{g}\right)$ and $\left(\lambda_{h}, \mu_{h}\right)$ to distinguish between $\lambda$ and $\mu$ parameters for the first and the second layers of the network.

In the first layer of the network, each source node splits its message into two parts, namely, functional and private parts, $W_{1}=\left(U_{1}^{(0)}, U_{1}^{(1)}\right)$ and $W_{2}=\left(U_{2}^{(0)}, U_{2}^{(1)}\right)$, where the functional parts, have the same rate, i.e., $\Upsilon_{1,0}=\Upsilon_{2,0}=\Upsilon_{0}$. Both 
transmitters use a common lattice code to encode their functional sub-messages into $\mathbf{x}_{1,0}=\psi\left(U_{1}^{(0)}\right)$ and $\mathbf{x}_{2,0}=\psi\left(U_{2}^{(0)}\right)$, where $\psi$ is the one-to-one encoding map induced by the lattice code. We define the partial-invertible function by

$\phi\left(U_{1}^{(0)}, U_{2}^{(0)}\right)=\psi^{-1}\left(\psi\left(U_{1}^{(0)}\right)+\psi\left(U_{2}^{(0)}\right)\right)=\psi^{-1}\left(\mathbf{x}_{1,0}+\mathbf{x}_{2,0}\right)$.

We denote the rates of the private sub-messages by $\Upsilon_{1}$ and $\Upsilon_{2}$, where $\Upsilon_{i}=R_{i}-\Upsilon_{0}$, for $i=1,2$. The goal is to encode and forward messages to $A$ and $B$ in such a way that $A$ can decode $U_{1}^{(1)}$ and $\phi\left(U_{1}^{(0)}, U_{2}^{(0)}\right)$, and $B$ can decode $U_{2}^{(0)}$ and $U_{2}^{(1)}$. Based on Lemma 3, this can be done provided that

$$
\begin{aligned}
\Upsilon_{0} & \leq\left(\frac{1}{2} \log \left(\lambda_{g}\right)-\frac{1}{2}\right)^{+} \\
\Upsilon_{0}+\Upsilon_{1} & \leq\left(\frac{1}{2} \log \left(g_{11}\right)-1\right)^{+} \\
\Upsilon_{0}+\Upsilon_{2} & \leq\left(\frac{1}{2} \log \left(g_{22}\right)-1\right)^{+} \\
\Upsilon_{0}+\Upsilon_{1}+\Upsilon_{2} & \leq\left(\frac{1}{2} \log \left(\mu_{g}\right)-\frac{3}{2}\right)^{+} .
\end{aligned}
$$

The second layer of the network is another Z-neutralization network with transmitters $A$ and $B$, and receivers $D_{1}$ and $D_{2}$. We use $V_{1}^{(0)}=\psi^{-1}\left(\psi\left(U_{1}^{(0)}\right)+\psi\left(U_{2}^{(0)}\right)\right), V_{1}^{(1)}=U_{1}^{(1)}$ as the functional and private messages of the first relay node, and $V_{2}^{(0)}=\psi^{-1}\left(-\mathbf{x}_{2,0}\right)=\psi^{-1}\left(-\psi\left(U_{2}^{(0)}\right)\right)$ and $V_{2}^{(1)}=U_{2}^{(1)}$ for the functional and private messages of second relay. Denoting the corresponding rates by $\Theta_{0}, \Theta_{1}$, and $\Theta_{2}$, we have

$$
\Theta_{i}=\Upsilon_{i}, \quad i=0,1,2 .
$$

The goal is to encode and send these messages to the destinations, such that $D_{1}$ can decode $\phi\left(V_{1}^{(0)}, V_{2}^{(0)}\right)$ and $V_{1}^{(1)}$, and $D_{2}$ can decode $V_{2}^{(0)}$ and $V_{2}^{(1)}$. Again, we use the achievable rate region proposed in Lemma 3

$$
\begin{aligned}
\Theta_{0} & \leq\left(\frac{1}{2} \log \left(\lambda_{h}\right)-\frac{1}{2}\right)^{+} \\
\Theta_{0}+\Theta_{1} & \leq\left(\frac{1}{2} \log \left(h_{11}\right)-1\right)^{+} \\
\Theta_{0}+\Theta_{2} & \leq\left(\frac{1}{2} \log \left(h_{22}\right)-1\right)^{+} \\
\Theta_{0}+\Theta_{1}+\Theta_{2} & \leq\left(\frac{1}{2} \log \left(\mu_{h}\right)-\frac{3}{2}\right)^{+} .
\end{aligned}
$$

Note that the first destination observes $\phi\left(V_{1}^{(0)}, V_{2}^{(0)}\right)$, which is equivalent to

$$
\begin{aligned}
\phi\left(V_{1}^{(0)}, V_{2}^{(0)}\right) & =\psi^{-1}\left(\psi\left(V_{1}^{(0)}\right)+\psi\left(V_{2}^{(0)}\right)\right) \\
& =\psi^{-1}\left(\psi\left(U_{1}^{(0)}\right)+\psi\left(U_{2}^{(0)}\right)-\psi\left(U_{2}^{(0)}\right)\right) \\
& =U_{1}^{(0)} .
\end{aligned}
$$

Therefore, combining it with $V_{1}^{(1)}=U_{1}^{(1)}$, the first destination node can decode $W_{1}$. The second destination node $D_{2}$ has $V_{2}^{(0)}$ and $V_{2}^{(1)}=U_{2}^{(1)}$, and can compute

$$
\psi^{-1}\left(-\psi\left(V_{2}^{(0)}\right)\right)=U_{2}^{(0)}
$$

and hence, it decodes $W_{2}$.

This scheme can reliably transmit the messages with rate pair in

$$
\begin{aligned}
& \mathcal{R}_{\text {ach }}^{\mathrm{GZZ}}=\left\{\left(R_{1}, R_{2}\right): \exists \Upsilon_{0}, \Upsilon_{1}, \Upsilon_{2}, \Theta_{0}, \Theta_{1}, \Theta_{2} \geq 0\right. \\
& R_{1}=\Theta_{0}+\Theta_{1} \\
& R_{2}=\Theta_{0}+\Theta_{2} \\
& \Theta_{i}=\Upsilon_{i}, \quad i=0,1,2 \\
& \Upsilon_{0} \leq\left(\frac{1}{2} \log \left(\lambda_{g}\right)-\frac{1}{2}\right)^{+} \\
& \Upsilon_{0}+\Upsilon_{1} \leq\left(\frac{1}{2} \log \left(g_{11}\right)-1\right)^{+} \\
& \Upsilon_{0}+\Upsilon_{2} \leq\left(\frac{1}{2} \log \left(g_{22}\right)-1\right)^{+} \\
& \Upsilon_{0}+\Upsilon_{1}+\Upsilon_{2} \leq\left(\frac{1}{2} \log \left(\mu_{g}\right)-\frac{3}{2}\right)^{+} \\
& \Theta_{0} \leq\left(\frac{1}{2} \log \left(\lambda_{h}\right)-\frac{1}{2}\right)^{+} \\
& \Theta_{0}+\Theta_{1} \leq\left(\frac{1}{2} \log \left(h_{11}\right)-1\right)^{+} \\
& \Theta_{0}+\Theta_{2} \leq\left(\frac{1}{2} \log \left(h_{22}\right)-1\right)^{+} \\
& \left.\Theta_{0}+\Theta_{1}+\Theta_{2} \leq\left(\frac{1}{2} \log \left(\mu_{h}\right)-\frac{3}{2}\right)^{+}\right\} \text {. }
\end{aligned}
$$

It only remains to apply Fourier-Motzkin elimination to project this region onto $\left(R_{1}, R_{2}\right)$ space. This gives us

$$
\begin{aligned}
& \mathcal{R}_{\mathrm{ach}}^{\mathrm{GZZ}}=\left\{\left(R_{1}, R_{2}\right)\right.: R_{1} \leq\left(\frac{1}{2} \log \left(g_{11}\right)-1\right)^{+} \\
& R_{1} \leq\left(\frac{1}{2} \log \left(h_{11}\right)-1\right)^{+}{ }^{+} \\
& R_{2} \leq\left(\frac{1}{2} \log \left(g_{22}\right)-1\right)^{+} \\
& R_{2} \leq\left(\frac{1}{2} \log \left(h_{22}\right)-1\right)^{+} \\
& R_{1}+R_{2} \leq\left(\frac{1}{2} \log \left(\mu_{g}\right)+\frac{1}{2} \log \left(\lambda_{h}\right)-\frac{3}{2}\right)^{+} \\
&\left.R_{1}+R_{2} \leq\left(\frac{1}{2} \log \left(\mu_{h}\right)+\frac{1}{2} \log \left(\lambda_{g}\right)-\frac{3}{2}\right)^{+}\right\} .
\end{aligned}
$$

Note that the RHS's of the sum-rate bounds depend on the order of the channel gains. For most of possible orderings, these two inequalities would be consequences of the individual rate bounds. For example, if $\lambda_{h}=h_{22}$, then the last bound is 
implied by the first and fourth bounds, since $\mu_{g} \geq g_{11}$. It can be shown in general that $\mathcal{R}_{\mathrm{ach}}^{\mathrm{GZZ}}$ is equivalent to

$$
\begin{aligned}
\mathcal{R}_{\mathrm{ach}}^{\mathrm{GZZ}}=\left\{\left(R_{1}, R_{2}\right)\right. & : R_{1} \leq\left(\frac{1}{2} \log \left(g_{11}\right)-1\right)^{+} \\
R_{1} & \leq\left(\frac{1}{2} \log \left(g_{22}\right)-1\right)^{+} \\
R_{2} & \leq\left(\frac{1}{2} \log \left(h_{11}\right)-1\right)^{+} \\
R_{2} & \leq\left(\frac{1}{2} \log \left(h_{22}\right)-1\right)^{+} \\
R_{1}+R_{2} & \leq\left(\frac{1}{2} \log \left(\mu_{g}\right)+\frac{1}{2} \log \left(h_{12}\right)-\frac{3}{2}\right)^{+} \\
R_{1}+R_{2} & \left.\leq\left(\frac{1}{2} \log \left(\mu_{h}\right)+\frac{1}{2} \log \left(g_{12}\right)-\frac{3}{2}\right)^{+}\right\} .
\end{aligned}
$$

Now, note that $g_{11} \geq 1, g_{12} \geq 1$, and $g_{22} \geq 1$. These imply

$$
\begin{aligned}
\frac{1}{2} \log & \left(1+g_{11}+g_{12}\right)+\frac{1}{2} \log \left(1+\frac{g_{22}}{g_{12}}\right) \\
& \leq \frac{1}{2} \log \left(3 \max \left\{g_{11}, g_{12}\right\}\right)+\frac{1}{2} \log \left(\frac{2 \max \left\{g_{12}, g_{22}\right\}}{g_{12}}\right) \\
& \leq \frac{1}{2} \log \left(\frac{\max \left\{g_{11}, g_{12}\right\} \cdot \max \left\{g_{22}, g_{12}\right\}}{g_{12}}\right)+\frac{1}{2} \log 6 \\
& \leq \frac{1}{2} \log \left(\mu_{h}\right)+\frac{1}{2} \log 6 .
\end{aligned}
$$

We also have

$$
\frac{1}{2} \log (1+x) \leq \frac{1}{2} \log (x)+\frac{1}{2}
$$

for all $x \geq 1$. Applying (B.38) and (B.39), we obtain the following achievable rate region, which is a subset of $\mathcal{R}_{\text {ach }}^{\mathrm{GZZ}}$.

$$
\begin{aligned}
& \mathcal{R}_{\mathrm{ach}, 2}^{\mathrm{GZZ}}=\left\{\left(R_{1}, R_{2}\right): R_{1} \leq\left(\frac{1}{2} \log \left(1+g_{11}\right)-\frac{3}{2}\right)^{+}\right. \\
& R_{2} \leq\left(\frac{1}{2} \log \left(1+g_{22}\right)-\frac{3}{2}\right)^{+} \\
& R_{1} \leq\left(\frac{1}{2} \log \left(1+h_{11}\right)-\frac{3}{2}\right)^{+} \\
& R_{2} \leq\left(\frac{1}{2} \log \left(1+h_{22}\right)-\frac{3}{2}\right)^{+} \\
& R_{1}+R_{2} \leq\left(\frac{1}{2} \log \left(1+g_{11}+g_{12}\right)\right. \\
&\left.+\frac{1}{2} \log \left(1+\frac{g_{22}}{g_{12}}\right)+\frac{1}{2} \log \left(1+h_{12}\right)-\frac{7}{2}\right)^{+} \\
& R_{1}+R_{2} \leq\left(\frac{1}{2} \log \left(1+h_{11}+h_{12}\right)\right. \\
&\left.\left.+\frac{1}{2} \log \left(1+\frac{h_{22}}{h_{12}}\right)+\frac{1}{2} \log \left(1+g_{12}\right)-\frac{7}{2}\right)^{+}\right\} .
\end{aligned}
$$

Therefore, for any rate pair $\left(R_{1}, R_{2}\right) \in \mathcal{R}^{\mathrm{GZZ}}$, the rate pair ( $R_{1}-\frac{7}{4}, R_{2}-\frac{7}{4}$ ) belongs to $\mathcal{R}_{\mathrm{ach}, 2}^{\mathrm{GZZ}}$ and, therefore, can be achieved using the proposed encoding scheme.

\section{APPENDIX C}

PROOF OF LEMMAS

A) Discussion of Example 4 in Section IV: The converse proof is fairly simple and follows from a similar argument we used to prove (GZS-1), (GZS-2), and (GZS-3) in Appendix A.

In the following we will present an encoding strategy which guarantees to achieve rate pair $\left(R_{1}-\frac{1}{2}, R_{2}-\frac{1}{2}\right)$, provided that $\left(R_{1}, R_{2}\right) \in \mathcal{R}^{Z}$. This gives us an approximate capacity characterization for the Gaussian $\mathrm{Z}$ network. In order to do this, we consider the following two cases.

Case A: $g_{12} \geq g_{22}$ : Assume $\left(R_{1}, R_{2}\right)$ be an achievable rate pair. Then, the first receiver $G_{1}$ is able to decode $W_{1}$ sent at rate $R_{1}$, and remove the signal associated to $W_{1}$ from its received signal. The remaining signal provides a higher SNR to decode $W_{2}$ than the signal received at $G_{2}$. Therefore, in this particular regime, the first receive would be able to decode both messages. Hence, we have a Gaussian multiple access channel from $F_{1}$ and $F_{2}$ to $G_{1}$, combined with a line network from $F_{2}$ to $G_{2}$. Therefore, the intersection of the rate regions of the Gaussian MAC and the line networks is simply achievable. That is

$$
\begin{aligned}
& \mathcal{R}_{\mathrm{ach}, \mathrm{A}}^{\mathrm{Z}}=\left\{\left(R_{1}, R_{2}\right): R_{1} \leq \frac{1}{2} \log \left(1+g_{11}\right)\right. \\
& R_{2} \leq\left.\frac{1}{2} \log \left(1+g_{12}\right), R_{1}+R_{2} \leq \frac{1}{2} \log \left(1+g_{11}+g_{12}\right)\right\} \\
& \bigcap \\
&\left\{\left(R_{1}, R_{2}\right): R_{2} \leq \frac{1}{2} \log \left(1+g_{22}\right)\right\} \\
&=\left\{\left(R_{1}, R_{2}\right): R_{1} \leq \frac{1}{2} \log \left(1+g_{11}\right)\right. \\
&\left.R_{2} \leq \frac{1}{2} \log \left(1+g_{22}\right), R_{1}+R_{2} \leq \frac{1}{2} \log \left(1+g_{11}+g_{12}\right)\right\} .
\end{aligned}
$$

Note that the individual rate bounds in $\mathcal{R}^{\mathrm{Z}}$ and $\mathcal{R}_{\text {ach,A }}^{\mathrm{Z}}$ are the same. Moreover, the difference between the sum rate bounds is bounded by

$$
\frac{1}{2} \log \left(1+\frac{g_{22}}{g_{12}}\right) \leq \frac{1}{2} \log (1+1)=\frac{1}{2}
$$

Therefore, the gap between each boundary point of $\mathcal{R}^{\mathrm{Z}}$ and $\mathcal{R}_{\text {ach,A }}^{\mathrm{Z}}$ is at most $\frac{1}{2}$ bit.

Case B: $g_{12} \leq g_{22}$ : The encoding scheme we introduce for this case is similar to Han-Kobayashi's scheme for 2-user interference channel. We first split the second message $W_{2}$ into the common and private parts, $W_{2}=\left(W_{2}^{c}, W_{2}^{p}\right)$, with rates $R_{2}^{c}$ and $R_{2}^{p}$, respectively, where $W_{2}^{c}$ can be decoded at both receivers and $W_{2}^{p}$ is only decodable at $G_{2}$. Sub-messages $W_{1}$, $W_{2}^{c}$, and $W_{2}^{p}$ are encoded by corresponding randomly generated Gaussian codes to $\mathbf{x}_{1}, \mathbf{x}_{2}^{c}$ and $\mathbf{x}_{2}^{p}$, and the resulting codewords are sent over the channel. 


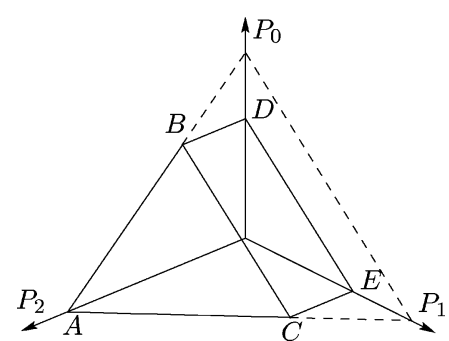

Fig. 18. Achievable rate region of the Z-neutralization network when $\eta=g_{11}$.

We allocate $\alpha_{p}=1 / g_{12}$ fraction of the transmission power available at $F_{2}$ to $W_{2}^{p}$, and the remaining power $\alpha_{c}=1-\alpha_{p}$ is allocated to $W_{2}^{c}$. Therefore, we have

$$
\mathbf{x}_{2}=\sqrt{\alpha_{c}} \mathbf{x}_{2}^{p}+\sqrt{\alpha_{c}} \mathbf{x}_{2}^{p}
$$

The first receiver, $G_{1}$, decodes $W_{1}$ and $W_{2}^{c}$ treating $W_{2}^{p}$ as noise. Therefore, the effective noise power received at $G_{1}$ would be $\mathbb{E}\left[\sqrt{g_{12} \alpha_{p}} x_{p}+z_{1}\right]^{2}=2$. According to the capacity region of Gaussian multiple access channel, this can be done provided that

$$
\begin{aligned}
R_{1} & \leq \frac{1}{2} \log \left(1+\frac{g_{11}}{2}\right) \\
R_{2}^{c} & \leq \frac{1}{2} \log \left(\frac{1+g_{12}}{2}\right) \\
R_{1}+R_{2}^{c} & \leq \frac{1}{2} \log \left(\frac{1+g_{11}+g_{12}}{2}\right) .
\end{aligned}
$$

The second decoder first decodes $W_{2}^{c}$ treating $W_{2}^{p}$ as noise. It then removes the corresponding codeword from the received signal, and decodes $W_{2}^{p}$. This can be done as long as

$$
\begin{aligned}
R_{2}^{c} & \leq \frac{1}{2} \log \left(\frac{1+g_{22}}{1+g_{22} / g_{12}}\right) \\
R_{2}^{p} & \leq \frac{1}{2} \log \left(1+\frac{g_{22}}{g_{12}}\right) .
\end{aligned}
$$

Note that we have two upper bounds for $R_{2}^{c}$. However, it is easy to show that $\frac{1+g_{22}}{1+g_{22} / g_{12}} \geq \frac{1+g_{12}}{2}$, for $1 \leq g_{12} \leq g_{22}$, and, therefore, the first bound dominates the second one. Using Fourier-Motzkin elimination to write the achievable region in terms of $R_{1}$ and $R_{2}=R_{2}^{c}+R_{2}^{p}$, and after some simplification, we get that the region

$$
\begin{aligned}
\mathcal{R}_{\text {ach, } \mathrm{B}}^{\mathrm{Z}} & =\left\{\left(R_{1}, R_{2}\right): R_{1} \leq \frac{1}{2} \log \left(1+g_{11}\right)-\frac{1}{2},\right. \\
R_{2} & \leq \frac{1}{2} \log \left(1+g_{22}\right)-\frac{1}{2}, \\
R_{1}+R_{2} & \left.\leq \frac{1}{2} \log \left(1+g_{11}+g_{12}\right)+\frac{1}{2} \log \left(1+\frac{g_{22}}{g_{12}}\right)-\frac{1}{2} \cdot\right\} .
\end{aligned}
$$

is achievable. Therefore, if $\left(R_{1}, R_{2}\right) \in \mathcal{R}^{\mathrm{Z}}$, then $\left(R_{1}-\frac{1}{2}, R_{2}-\right.$ $\left.\frac{1}{2}\right)$ is achievable.

B) Proof of Lemma 1 in Section V: The following achievability scheme simply uses superposition encoding of sub-messages at $F_{2}$, and a successively decode and cancel strategy at $G_{1}$ and $G_{2}$. We use a random codebook with a proper number of codewords, generated according to a zero-mean unit-variance Gaussian distribution for each message. A proper power allocation for the messages at the transmitters allow the decoders to apply a decode and cancel strategy. We denote the codeword corresponding to the message $U_{i}^{(j)}$ by $\mathbf{x}_{i, j}$, and the power allocated to this message by $\alpha_{i, j}$.

The available power at $F_{2}$ can be arbitrarily allocated to its sub-messages. In particular, we choose the power coefficients so that they satisfy $\alpha_{2,2} \leq 1 / g_{22}, \alpha_{2,3} \leq 1 / g_{12}$, and $\alpha_{2,1}=1-$ $\alpha_{2,2}-\alpha_{2,3}$. In the decoding part, $G_{1}$ and $G_{2}$ treat $U_{2}^{(3)}$ and $U_{2}^{(2)}$, respectively, as noise. Therefore, the total noise at $G_{1}$ and $G_{2}$ would be $\tilde{\mathbf{z}}_{1}=\sqrt{g_{12} \alpha_{2,3}} \mathbf{x}_{2,3}+\mathbf{z}_{1}$ and $\tilde{\mathbf{z}}_{2}=\sqrt{g_{22} \alpha_{2,2}} \mathbf{x}_{2,2}+$ $\mathbf{z}_{2}$. However, the effective noise power cannot exceed 2 since $\mathbb{E}\left[g_{12} \alpha_{2,3}+1\right] \leq 2$ and $\mathbb{E}\left[g_{22} \alpha_{2,2}+1\right] \leq 2$.

The receiver $F_{1}$ observes a Gaussian multiple access channel (with noise power upper bounded by 2 ), where $U_{1}^{(1)}$ is sent by one user, and $\left(U_{2}^{(1)}, U_{2}^{(2)}\right)$ is sent by the other user. The bounds in (11)-(14) guarantee that these rates are achievable over the multiple access channel.

On the other hand, the channel from $F_{2}$ to $G_{2}$ is Gaussian point-to-point channel with modified additive noise. Therefore, any total rate not exceeding its capacity can be reliably transmitted. This is condition is fulfilled here since $\Upsilon_{2,1}+\Upsilon_{2,3}$ satisfies (16). Finally, the bound on the power allocated to $U_{2}^{(3)}$ upper bounds its rate as in (15).

C) Proof of Lemma 2 in Section V: Again, the achievability scheme we propose for the Gaussian $S$ interference network (illustrated in Fig. 11) is based on superposition coding, and a successively decode and cancel decoding strategy, such that the requirements of the problem are fulfilled. A proper power allocation is required to guarantee achievability of the rate tuples mentioned in this lemma.

Note that $G_{1}$ does not decode $V_{2}^{(2)}$ and $V_{2}^{(4)}$, and treats them as noise. We choose the total fraction of power allocated to $V_{2}^{(2)}$ and $V_{2}^{(4)}$ to be at most $1 / h_{11}$, that is $\alpha_{2,2}+\alpha_{2,4} \leq 1 / h_{11}$. Therefore, the total noise power received at $G_{1}$ is upper bounded as $\mathbb{E}\left[h_{11}\left(\alpha_{2,2}+\alpha_{2,4}\right)+1\right] \leq 2$.

Similarly, $V_{1}^{(2)}$ is treated as noise at $G_{2}$. By bounding the fraction of power allocated to this sub-message, we can upper bound the effective noise power observed at $G_{2}$ by $\mathbb{E}\left[h_{11}\left(\alpha_{2,2}+\alpha_{2,4}\right)+1\right] \leq 2$.

The point-to-point Gaussian channel from $F_{1}$ to $G_{1}$ can support any sum-rate below its capacity as in (17). Moreover, $\Theta_{1,2}$ is bounded above since its allocated power does not exceed $1 / h_{12}$.

On the other hand, we have a Gaussian multiple access channel from $F_{1}$ and $F_{2}$ to $G_{2}$, with total noise power not exceeding 2. The bounds in (19)-(22) guarantee that the desired rates belong to the capacity region of this channel, and, therefore, they are achievable. We skip the details of power allocation here, but we point out that the achievability of the region is a consequence of the Gaussian multiple access rate region achievability.

D) Proof of Lemma 3 in Section V: In this part we show that any rate tuple satisfying (25)-(28) is achievable. The main idea of this proof can be summarized as follows. 
- Use a common codebook with group structure, such as lattice codes, for $W_{1}^{(0)}$ and $W_{2}^{(0)}$, which maps them to $\mathbf{x}_{1,0}$ and $\mathbf{x}_{2,0}$

- Choose a proper power allocation for $\mathbf{x}_{1,0}$ and $\mathbf{x}_{2,0}$ such that they get received at $G_{1}$ at the same power level; More precisely, denoting their power allocation by $\alpha_{0}$ and $\beta_{0}$, they should satisfy $g_{11} \alpha_{0}=g_{12} \beta_{0}$. This condition guarantees that the two lattice points get scaled by the same factor, and, therefore, the result is still a lattice point on the scaled lattice and can be decoded as long as enough signal to noise ratio is provided.

- Use random Gaussian codebooks to encode the private sub-messages to $\mathbf{x}_{1,1}$ and $\mathbf{x}_{1,2}$, and use proper power allocation, $\alpha_{1}$ and $\beta_{1}$.

The first receiver $G_{1}$ needs to decode the partial-invertible $\phi$ which we define as

$$
\begin{aligned}
\phi\left(W_{1}^{(0)}, W_{2}^{(0)}\right) & =\psi^{-1}\left(\psi\left(W_{1}^{(0)}\right)+\psi\left(W_{1}^{(0)}\right)\right) \\
& =\psi^{-1}\left(\mathbf{x}_{1,0}+\mathbf{x}_{2,0}\right)
\end{aligned}
$$

where $\psi$ is the one-to-one encoding function which maps the functional messages to the common lattice codebook. Note that the group structure of the code impels that $\mathbf{x}_{1,0}+\mathbf{x}_{2,0}$ is still a valid codeword. It is easy to check that this function is partialinvertible.

Let us define

$$
\eta=\min \left\{g_{11}, g_{12}, g_{22}, \frac{g_{11} g_{22}}{g_{12}}\right\} \text {. }
$$

Depending on the minimizer in $\eta$, we identify four cases. In each case, the achievable rate region is a polytope, with a certain number of corner points. It suffices to show the achievability only for the cornet points, since a standard time-sharing argument guarantees achievability for the rest of the region.

The proof details for each corner point includes message splitting, and power allocation for sub-messages such that the decoders be able to decode corresponding messages. In the following we describe this strategy in details for the case where $\eta=g_{11}$. The extension of this method for other cases is straight-forward, and, therefore, we skip it here to sake of brevity.

Case I. $\eta=g_{11}$ : It is clear from the definition of $\eta$ that in this case $g_{11} \leq g_{12} \leq g_{22}$, and, therefore, $\lambda=g_{11}$ and $\mu=g_{22}$. Hence, the desired region is characterized by all non-negative rate tuples $\left(P_{0}, P_{1}, P_{2}\right)$ satisfying

$$
\begin{aligned}
P_{0}+P_{1} & \leq \frac{1}{2} \log \left(g_{11}\right) \\
P_{0}+P_{1}+P_{2} & \leq \frac{1}{2} \log \left(g_{22}\right) .
\end{aligned}
$$

This rate region is illustrated in Fig. 18. It suffices to show that the corner points $A, B$ and $C$ are achievable, since the points $D$ and $E$ are degenerated from $B$ and $C$, respectively.

- $A:\left(P_{0}, P_{1}, P_{2}\right)=\left(0,0, \frac{1}{2} \log \left(g_{22}\right)-\frac{3}{2}\right)$

The encoding strategy for this corner point is fairly simple. The second transmitter uses all its available power to send $W_{2}^{(1)}$, while the first transmitter keeps silent. That is, $\mathbf{x}_{1}=$
0 and $\mathbf{x}_{2}=\mathbf{x}_{2,1}$. The first decoder has nothing to decode, and the second one can decode $\mathbf{x}_{2}$ from $\mathbf{y}_{2}$ as long as $P_{2} \leq \frac{1}{2} \log \left(1+g_{22}\right)$. It is clear that in particular $P_{2}=$ $\frac{1}{2} \log \left(g_{22}\right)-\frac{3}{2}$ is achievable.

- $B:\left(P_{0}, P_{1}, P_{2}\right)=\left(\frac{1}{2} \log \left(g_{11}\right)-1,0, \frac{1}{2} \log \left(g_{22}\right)-\frac{1}{2} \log \left(g_{11}\right)-\frac{1}{2}\right)$ The first encoder sends its lattice codeword with power allocation $\alpha_{0}=\left(g_{11}-1\right) / g_{11}$. The second encoder splits its private message into $W_{2}^{(1)}=\left(W_{2}^{(1,1)}, W_{2}^{(1,2)}\right)$ of rates $P_{2,1}$ and $P_{2,2}$ where $P_{2}=P_{2,1}+P_{2,2}$. Then it sends

$$
\mathbf{x}_{2}=\sqrt{\beta_{1,1}} \mathbf{x}_{2,1,1}+\sqrt{\beta_{0}} \mathbf{x}_{2,0}+\sqrt{\beta_{1,2}} \mathbf{x}_{2,1,2}
$$

where the power allocation coefficients are fixed to be $\beta_{1,2}=1 / g_{12}, \beta_{0}=\left(g_{11}-1\right) / g_{12}$, and $\beta_{1,1}=1-\beta_{0}-\beta_{1,2}$. The signal received at the destinations are

$$
\begin{aligned}
\mathbf{y}_{1}= & \sqrt{g_{11}} \mathbf{x}_{1}+\sqrt{g_{12}} \mathbf{x}_{2}+\mathbf{z}_{1} \\
= & \sqrt{g_{12}-g_{11}} \mathbf{x}_{2,1,1}+\sqrt{g_{11}-1}\left[\mathbf{x}_{1,0}+\mathbf{x}_{2,0}\right] \\
& +\mathbf{x}_{2,1,2}+\mathbf{z}_{1} \\
\mathbf{y}_{2}= & \sqrt{g_{22}} \mathbf{x}_{2}+\mathbf{z}_{2} \\
= & \sqrt{\frac{g_{22}\left(g_{12}-g_{11}\right)}{g_{12}}} \mathbf{x}_{2,1,1}+\sqrt{\frac{g_{22}\left(g_{11}-1\right)}{g_{12}}} \mathbf{x}_{2,0} \\
& +\sqrt{\frac{g_{22}}{g_{12}}} \mathbf{x}_{2,1,2}+\mathbf{z}_{2} .
\end{aligned}
$$

The first node decode and cancel $\mathbf{x}_{2,1,1}, \tilde{\mathbf{x}}_{0}=\mathbf{x}_{1,0}+\mathbf{x}_{2,0}$, and $\mathbf{x}_{2,1,2}$ in order, while the second one performs the same decoding for $\mathbf{x}_{2,1,1}, \mathbf{x}_{2,0}$, and $\mathbf{x}_{2,1,2}$. It is easy to show that the rates $P_{2,1}=\frac{1}{2} \log \left(g_{12} / g_{11}\right)-0.5, P_{0}=$ $\frac{1}{2} \log \left(g_{11}\right)-1$, and $P_{2,2}=\frac{1}{2} \log \left(g_{22} / g_{12}\right)$ are achievable, which implies the private rates $P_{2}=P_{2,1}+P_{2,2}=$ $\frac{1}{2} \log \left(g_{22} / g_{11}\right)-\frac{1}{2}$ for the second transmitter.

- $\mathrm{C}$ $\left(0, \frac{1}{2} \log \left(g_{11}\right)-1, \frac{1}{2} \log \left(g_{22}\right)-\frac{1}{2} \log \left(g_{11}\right)-\frac{1}{2}\right)$

For this rate tuple, the rate of the functional message is zero. The second transmitter splits its private message similar to that of corner point $B$. The transmission power is distributed between among the sub-message as $\alpha_{0}=0$, $\alpha_{1}=1, \beta_{1,2}=1 / g_{12}, \beta_{0}=0$, and $\beta_{1,1}=1-\beta_{1,2}$. A similar argument to that of corner point $B$ shows that the rates $P_{2,1}=\frac{1}{2} \log \left(g_{12} / g_{11}\right)-0.5, P_{1}=\frac{1}{2} \log \left(g_{11}\right)-1$, and $P_{2,2}=\frac{1}{2} \log \left(g_{22} / g_{12}\right)$ are achievable, which implies the achievability of the rate point $C$.

E) Proof of Lemma 4 in Section VI-A: The entropy of any random variable can be simply upper bounded by the logarithm of its alphabet size. In particular, we have

$$
H\left(G_{1} X\right) \leq \log \left|\left\{G_{1} X: X \in \mathbb{F}_{2}^{q}\right\}\right|=\log 2^{\operatorname{rank}\left(G_{1}\right)}=\operatorname{rank}\left(G_{1}\right) .
$$

Note that this bound is tight and can be achieved by uniform distribution on $X$. In order to prove the second inequality, we can write

$$
\begin{aligned}
H\left(G_{1} X \mid G_{2} X\right) & =\sum_{x \in \mathbb{F}_{2}^{q}} \operatorname{Pr}\left(G_{2} X=x\right) H\left(G_{1} X \mid G_{2} X=x\right) \\
& \leq \max _{x \in \operatorname{img}\left(G_{2}\right)} H\left(G_{1} X \mid G_{2} X=x\right)
\end{aligned}
$$


where $\operatorname{img}\left(G_{2}\right)$ is the image space of the matrix $G_{2}$. The linear structure of the underlying spaces implies that $H\left(G_{1} X \mid G_{2} X=\right.$ $x)$ is the same for every $x \in \operatorname{img}\left(G_{2}\right)$. Therefore

$$
\begin{aligned}
H\left(G_{1} X \mid G_{2} X\right) & \leq H\left(G_{1} X \mid G_{2} X=0\right) \\
& \leq \log \left|\left\{G_{1} X: G_{2} X=0\right\}\right| \\
& =\left|\operatorname{ker}\left(G_{2}\right)\right|-\left|\operatorname{ker}\left(G_{1}\right) \cap \operatorname{ker}\left(G_{2}\right)\right| \\
& =\operatorname{rank}\left[\begin{array}{l}
G_{1} \\
G_{2}
\end{array}\right]-\operatorname{rank}\left(G_{2}\right) .
\end{aligned}
$$

where $\operatorname{ker}\left(G_{1}\right)=\left\{X: G_{1} X=0\right\}$ is the kernel space of $G_{1}$.

F) Proof of Lemma 5 in Section VI-B: Let $\left(R_{1}, R_{2}\right) \in \mathcal{R}^{\mathrm{DZS}}$ be an arbitrary rate pair which satisfies (DZS-1)-(DZS-10). In particular $R_{1} \leq \min \left\{m_{11}, n_{11}\right\}$. We claim that $\left(R_{1}, R_{2}\right) \in \mathcal{R}_{1}^{\mathrm{DZS}}(t) \times \mathcal{R}_{2}^{\mathrm{DZS}}(t)$ for $t=R_{1}$, and, therefore, $\left(R_{1}, R_{2}\right)$ is achievable using network decomposition. In order to do this we have to show that any $R_{2}$ satisfying (DZS-1)-(DZS-10), fulfills the constraints in the definition of $\mathcal{R}_{2}^{\mathrm{DZS}}\left(R_{1}\right)$.

Using (DZS-2) and (DZS-3), we have

$R_{2}$

$\leq \min \left(\max \left(m_{11}, m_{12}\right)+\left(m_{22}-m_{12}\right)^{+}-R_{1}, \max \left(m_{12}, m_{22}\right)\right)$

$=\min \left(\max \left(m_{11}, m_{12}\right)-R_{1}, m_{12}\right)+\left(m_{22}-m_{12}\right)^{+}$

$=m_{12}^{\prime}\left(R_{1}\right)+\left(m_{22}-m_{12}\right)^{+}$

$\leq m_{12}^{\prime}\left(R_{1}\right)+\left(m_{22}^{\prime}\left(R_{1}\right)-m_{12}^{\prime}\left(R_{1}\right)\right)^{+}$

$=\max \left(m_{12}^{\prime}\left(R_{1}\right), m_{22}^{\prime}\left(R_{1}\right)\right)$

where in (C.11) we have used the fact that

$$
(\min (a, b)-\min (c, d))^{+} \geq \min \left((a-c)^{+},(b-d)^{+}\right) .
$$

Moreover, since $R_{2}$ satisfies (DZS-3), (DZS-5), and (DZS-8), we have

$$
\begin{aligned}
R_{2} \leq & \min \left(\max \left(m_{11}, m_{12}\right)+\left(m_{22}-m_{12}\right)^{+}-R_{1}\right. \\
& \left.m_{22}+\max \left(n_{11}, n_{21}\right)-R_{1}, m_{22}+n_{21}\right) \\
\leq & \min \left(\max \left(m_{11}, m_{12}\right)+\left(m_{22}-m_{12}\right)^{+}-R_{1}, m_{22}\right) \\
& +\min \left(\max \left(n_{11}, n_{21}\right)-R_{1}, n_{21}\right) \\
= & m_{22}^{\prime}\left(R_{1}\right)+n_{21}^{\prime}\left(R_{1}\right)
\end{aligned}
$$

where (C.13) holds since

$$
\min (a, b)+\min (c, d) \geq \min (a, b+c, b+d)
$$

for non-negative $a, b, c$, and $d$.

In order to show that the third constraint is satisfied, we can start with (DZS-4), (DZS-6), and (DZS-10)

$$
\begin{aligned}
R_{2} \leq & \min \left(\max \left(m_{11}, m_{12}\right)+n_{22}+-R_{1}, m_{12}+n_{22}\right. \\
& \left.\max \left(n_{11}, n_{21}\right)+\left(n_{22}-n_{21}\right)^{+}-R_{1}\right) \\
\leq & \min \left(\max \left(m_{11}, m_{12}\right)+-R_{1}, m_{12}\right) \\
& +\min \left(\max \left(n_{11}, n_{21}\right)+\left(n_{22}-n_{21}\right)^{+}-R_{1}, n_{22}\right) \\
= & m_{12}^{\prime}\left(r_{1}\right)+n_{22}^{\prime}\left(r_{1}\right) .
\end{aligned}
$$

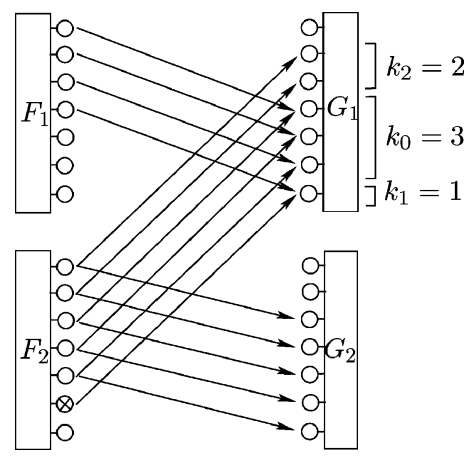

Fig. 19. Deterministic Z-neutralization network. The upper 2 sub-nodes in $G_{1}$ are only connected to $F_{2}$, and, therefore $k_{2}=2$. The next 3 sub-nodes receive information from both $F_{1}$ and $F_{2}$, and hence, $k_{0}=3$. Although the lowest sub-node is also connected to both transmitters, it only receives information from $F_{1}$ since $F_{2}$ keeps silent on its sub-nodes below $n_{22}$.

Finally, using (DZS-8) and (DZS-10), we have

$$
\begin{aligned}
R_{2} & \leq \min \left(\max \left(n_{11}, n_{21}\right)+\left(n_{22}-n_{21}\right)^{+}-r_{1}, \max \left(n_{21}, n_{22}\right)\right) \\
& =\min \left(\max \left(n_{11}, n_{21}\right)-r_{1}, n_{21}\right)+\left(n_{22}-n_{21}\right)^{+} \\
& =n_{21}^{\prime}\left(r_{1}\right)+\left(n_{22}-n_{21}\right)^{+} \\
& \leq n_{21}^{\prime}\left(r_{1}\right)+\left(n_{22}^{\prime}\left(r_{1}\right)-n_{21}^{\prime}\left(r_{1}\right)\right)^{+} \\
& =\max \left(n_{21}^{\prime}\left(r_{1}\right), n_{22}^{\prime}\left(r_{1}\right)\right) .
\end{aligned}
$$

Putting inequalities in (C.12) and (C.14)-(C.16) together shows that $R_{2} \in \mathcal{R}_{2}^{\mathrm{DZS}}\left(R_{1}\right)$, and completes the proof.

G) Proof of Lemma 6 in Section VII-B: The coding strategy we present here is based a network decomposition, where the sub-nodes and the links of the deterministic Z-interference network are partitioned into two disjoint sets. We analyze the rate region of each network, and derive an achievable rate region for the original network based on this analysis.

We just point out here that in this coding strategy, the second sender $F_{2}$, never sends a bit on a sub-node which is not received at $G_{2}$, even if $n_{12}>n_{22}$.

The first partition of the network $\mathcal{N}_{1}$, consists of those subnodes in $G_{1}$ which are connected to one of the top $m_{11}$ subnodes of $F_{1}$ and one of the top $m_{22}$ sub-nodes of $F_{2}$. All the subnodes in the network which are related to (see Definition 3 ) any of these sub-nodes also belong to the first network partition. The remaining nodes and link form the second part of the network $\mathcal{N}_{2}$. It is clear that these two networks are node-disjoint, and do not cause interference on each other.

We first characterize the number sub-nodes in $G_{1}$ which belong to $\mathcal{N}_{1}$, by determining whether each of them can receive a bit from $F_{1}, F_{2}$, or both of them. We denote the number of levels in $G_{1}$ which are only connected to a transmitting level in $F_{1}$ by $k_{1}$. Similarly, the number of those only connected to a a transmitting level (the top $\min \left(n_{12}, n_{22}\right)$ ) in $F_{2}$ by $k_{2}$. Finally, $k_{0}$ denotes the number of levels which are connected to transmitting levels of both $F_{1}$ and $F_{2}$ (see Fig. 19).

First, we derive $k_{0}$. Enumerate the levels of $G_{1}$ from 1 (for the highest) to $q$ (for the lowest). Let $j$ be the index of a sub-node in $G_{1}$ belong to $\mathcal{N}_{1}$, i.e., it receives bits from both $F_{1}$ and $F_{2}$. Its neighbors in $F_{1}$ and $F_{2}$ (if there is any) are indexed by $j+n_{11}-q$ and $j+n_{12}-q$, respectively. Therefore, $j$ belongs to $\mathcal{N}_{1}$ if 
and only if $1 \leq j+n_{11}-q \leq n_{11}$ and $1 \leq j+n_{12}-$ $q \leq \min \left(n_{12}, n_{22}\right)$. Therefore, the number of such sub-nodes is given by

$$
\begin{aligned}
k_{0} & =\left[\min \left\{q, q-n_{12}+n_{22}\right\}-\max \left\{q-n_{11}, q-n_{12}\right\}\right]^{+} \\
& =\min \left\{n_{11}, n_{12}, n_{22},\left(n_{11}+n_{22}-n_{12}\right)^{+}\right\} .
\end{aligned}
$$

It is clear from the definition of $k_{0}$ that the remaining $n_{11}-k_{0}$ lowest levels of $G_{1}$ are only connected to sub-nodes of $F_{1}$, and hence, $k_{1}=n_{11}-k_{0}$. Similarly, $\min \left\{n_{12}, n_{22}\right\}$ sub-nodes in $G_{1}$ are receiving information from $F_{2}$, where $k_{0}$ of them are also connected to $F_{1}$. Therefore, the remaining sub-nodes are only connected to $G_{2}$. Thus, $k_{2}=\min \left\{n_{12}, n_{22}\right\}-k_{0}$.

We partition the network into two parts: The first part consists of the $k_{0}$ sub-nodes of $G_{1}$ connected to both $F_{1}$ and $F_{2}$, and subnodes connected to them. The remaining sub-nodes form the second partition of the network. We characterize the achievable tuples for each, denoted by $\left(Q_{0}^{\prime}, Q_{1}^{\prime}, Q_{2}^{\prime}\right)$ and $\left(Q_{0}^{\prime \prime}, Q_{1}^{\prime \prime}, Q_{2}^{\prime \prime}\right)$, respectively. The fact that these two partitions are isolated allows us to conclude that the summation of such achievable tuples is also achievable for the original network.

Consider the first partition of the network. It is clear that any of the $k_{0}$ levels of $G_{1}$ connected to both $F_{1}$ and $F_{2}$ and can be used to communicate a functional bit, since $G_{1}$ naturally receives the xor of the transmitting bits. On the other hand, such sub-node can be used to communicate one private bit from any of $F_{1}$ or $F_{2}$ to $G_{1}$ by keeping the other one silent. Therefore, any rate tuple satisfying

$$
Q_{0}^{\prime}+Q_{1}^{\prime}+Q_{2}^{\prime} \leq k_{0}
$$

is achievable.

The non-interfered links of the second partition of the network can be used to send private bits from the transmitters to $G_{1}$ simultaneously. Moreover, each transmitter can use one of its non-interfering sub-nodes to send a functional bit to $G_{1}$, and then, $G_{1}$ computes their xor, after receiving them separately. This can provide up to $\min \left\{k_{1}, k_{2}\right\}$ new functional bits for $G_{1}$. Moreover, the lower $\left(n_{22}-n_{12}\right)^{+}$sub-nodes of $F_{2}$ which are connected to $G_{2}$ but not to $G_{1}$ can be used to send private bits to $G_{2}$ without causing any interference at $G_{1}$.

Hence, this strategy can transmit any rate tuple satisfying

$$
\begin{aligned}
Q_{0}^{\prime \prime} & \leq \min \left\{k_{1}, k_{2}\right\} \\
Q_{0}^{\prime \prime}+Q_{1}^{\prime \prime} & \leq k_{1} \\
Q_{0}^{\prime \prime}+Q_{2}^{\prime \prime} & \leq k_{2}+\left(n_{22}-n_{12}\right)^{+} .
\end{aligned}
$$

Summing up the rates achieved on each partition of the network, we have arrive at $Q_{i}=Q_{i}^{\prime}+Q_{i}^{\prime \prime}$ for $i=0,1,2$, where $\left(Q_{0}^{\prime}, Q_{1}^{\prime}, Q_{2}^{\prime}\right)$ 's and $\left(Q_{0}^{\prime \prime}, Q_{1}^{\prime \prime}, Q_{2}^{\prime \prime}\right)$ satisfy (C.18) and (C.19), respectively. It only remains to apply the Fourier-Motzkin elimination to project the rate region on the $\left(Q_{0}, Q_{1}, Q_{2}\right)$ space. This gives us

$$
\begin{aligned}
Q_{0} & \leq k_{0}+\min \left\{k_{1}, k_{2}\right\} \\
Q_{0}+Q_{1} & \leq k_{0}+k_{1} \\
Q_{0}+Q_{2} & \leq k_{0}+k_{2}+\left(n_{22}-n_{12}\right)^{+} \\
Q_{0}+Q_{1}+Q_{2} & \leq k_{0}+k_{1}+k_{2}+\left(n_{22}-n_{12}\right)^{+} \text {.(C.20) }
\end{aligned}
$$

Some simple manipulations show that the RHS's of the inequalities in (C.20) are the same as that claimed in the lemma.

H) Proof of Lemma 7 in Appendix A.1: As mentioned before, we will use the Fano's inequality in order to prove this lemma. We have

$$
\begin{aligned}
\ell R_{1} & =H\left(W_{1}\right)=I\left(W_{1} ; y_{1}^{\ell}\right)+H\left(W_{1} \mid y_{1}^{\ell}\right) \\
& \leq I\left(W_{1} ; y_{1}^{\ell}\right)+\ell e_{\ell} \\
& \leq I\left(x_{1}^{\ell} ; y_{1}^{\ell}\right)+\ell e_{\ell},
\end{aligned}
$$

where (C.21) is implied by the Fano's inequality, and in (C.22) we used the data processing inequality for the Markov chain $W_{1} \leftrightarrow x_{1}^{\ell} \leftrightarrow y_{1}^{\ell}$. Note that where $\varepsilon_{\ell} \rightarrow 0$ as $\ell$ grows. The proofs of the other two inequalities follow the same lines, and we skip them to sake of brevity.

I) Proof of Lemma 8 in Appendix B.1: Note that $Z$ is independent of everything else, and $X_{1}$ and $X_{2}$ are conditionally independent. Without loss of generality we can also assume that $\mu_{i}(\gamma)=\mathbb{E}\left[X_{i} \mid \Gamma=\gamma\right]=0$ for $\forall \gamma$ (otherwise for any given $\Gamma=\gamma$, we can shift $X_{i}$ by $\mu_{i}(\gamma)$, while the entropy does not change). Let $\mathbb{E}\left[X_{i}^{2} \mid \Gamma=\gamma\right]=\sigma_{i}^{2}(\gamma)$ for $i=1,2$. Therefore, the conditional variance of $Y$ can be bounded as

$\mathbb{E}\left[Y^{2} \mid \Gamma=\gamma\right]=\mathbb{E}\left[\left(X_{1}+X_{2}+Z\right)^{2} \mid \Gamma=\gamma\right]=\sigma_{1}^{2}(\gamma)+\sigma_{2}^{2}(\gamma)+1$.

Therefore

$$
\begin{aligned}
h(Y \mid \Gamma) & =\mathbb{E}_{\Gamma}[h(Y \mid \Gamma=\gamma)]=\mathbb{E}_{\Gamma}\left[h\left(X_{1}+X_{2}+Z \mid \Gamma=\gamma\right)\right] \\
& \leq \mathbb{E}_{\Gamma}\left[\log 2 \pi e\left(\sigma_{1}^{2}(\gamma)+\sigma_{2}^{2}(\gamma)+1\right)\right] \\
& \leq \log 2 \pi e\left(\mathbb{E}_{\Gamma}\left[\sigma_{1}^{2}(\gamma)+\sigma_{2}^{2}(\gamma)+1\right]\right) \\
& =\log 2 \pi e\left(\sigma_{1}^{2}+\sigma_{2}^{2}+1\right)
\end{aligned}
$$

where in (C.24) we have used the fact that Gaussian random variable has the maximum differential entropy among all random variables with the same variance, and (C.25) follows from the concavity of the function $\log (\cdot)$. Finally, (C.26) is just the tower property, $\mathbb{E}_{\Gamma}\left[\mathbb{E}\left[X_{i}^{2} \mid \Gamma\right]\right]=\mathbb{E}\left[X_{i}^{2}\right]$.

\section{REFERENCES}

[1] A. Schrijver, Theory of Linear and Integer Programming. Hoboken, NJ: Wiley, 1998.

[2] T. S. Han and K. Kobayashi, "A new achievable rate region for the interference channel," IEEE Trans. Inf. Theory, vol. IT-27, no. 1, pp. 49-60, Jan. 1981.

[3] R. H. Etkin, D. Tse, and H. Wang, "Gaussian interference channel capacity to within one bit," IEEE Trans. Inf. Theory, vol. 54, no. 12, pp. 5534-5562, Dec. 2008

[4] A. S. Avestimehr, S. N. Diggavi, and D. N. C. Tse, "Wireless network information flow: A deterministic approach," IEEE Trans. Inf. Theory, to be published.

[5] T. Cover and A. E. Gamal, "Capacity theorems for the relay channel," IEEE Trans. Inf. Theory, vol. IT-25, no. 5, pp. 572-584, Sep. 1979.

[6] A. Avestimehr, S. Diggavi, and D. Tse, "A deterministic approach to wireless relay networks," presented at the Allerton Conf. Communication, Control, and Computing, Sep. 2007.

[7] K. Gomadam and S. A. Jafar, "The effect of noise correlation in amplifyand-forward relay networks," IEEE Trans. Inf. Theory, vol. 55, no. 2, pp. 731-745, Feb. 2009.

[8] P. Gupta and P. Kumar, "The capacity of wireless networks," IEEE Trans. Inf. Theory, vol. 46, no. 2, pp. 388-404, Mar. 2000.

[9] A. Ozgur, O. Leveque, and D. Tse, "Hierarchical cooperation achieves optimal capacity scaling in ad hoc networks," IEEE Trans. Inf. Theory, vol. 53, no. 10, pp. 3549-3572, Oct. 2007. 
[10] M. Franceschetti, M. Migliore, and P. Minero, "The capacity of wireless networks: Information-theoretic and physical limits," IEEE Trans. Inf. Theory, vol. 55, no. 8, pp. 3413-3424, Aug. 2009.

[11] V. Prabhakaran and P. Viswanath, "Interference channel with destination cooperation," presented at the IEEE Int. Symp. Information Theory (ISIT), Seoul, Korea, Jun. 2009.

[12] C. Suh and D. Tse, "Symmetric feedback capacity of the gaussian interference channel to within one bit," presented at the IEEE Int. Symp. Information Theory (ISIT), Seoul, Korea, Jun. 2009.

[13] G. Bresler and D. Tse, "The two-user Gaussian interference channel: A deterministic view," Eur. Trans. Telecommun., vol. 19, pp. 333-354, Jun. 2008.

[14] V. Cadambe and S. Jafar, "Interference alignment and degrees of freedom of the K-user interference channel," IEEE Trans. Inf. Theory, vol. 54, no. 8, pp. 3425-3441, Aug. 2008.

[15] M. Maddah-Ali, A. Motahari, and A. Khandani, "Communication over MIMO X channels: Interference alignment, decomposition, and performance analysis," IEEE Trans. Inf. Theory, vol. 54, no. 8, pp. 3457-3470, Aug. 2008.

[16] T. M. Cover and J. Thomas, Elements of Information Theory. Hoboken, NJ: Wiley, 1991.

[17] S. Mohajer, S. N. Diggavi, C. Fragouli, and D. N. C. Tse, "Transmission techniques for relay-interference networks," presented at the Allerton Conf. Communication, Control, and Computing, Sep. 2008.

[18] A. Avestimehr, S. Diggavi, and D. Tse, "Wireless network information flow," presented at the Allerton Conf. Communication, Control, and Computing, Sep. 2007.

[19] S. Mohajer, S. Diggavi, C. Fragouli, and D. N. C. Tse, "Capacity of deterministic z-chain relay-interference network," in Proc. IEEE Information Theory Workshop, Volos, Greece, 2009, pp. 331-335.

Soheil Mohajer (M'10) received the B.S. degree in electrical engineering from the Sharif University of Technology, Tehran, Iran, in 2004. He received the M.S. degree in communication systems in 2005 and the Ph.D. degree in 2010, both from Ecole Polytechnique Fédérale de Lausanne (EPFL), Lausanne, Switzerland.

Since October 2010, he has been a Postdoctoral Researcher at Princeton University, Princeton, NJ. His fields of interests are multiuser information theory, network coding theory, and wireless communication.

Suhas N. Diggavi (S'93-M'98) received the B.Tech. degree in electrical engineering from the Indian Institute of Technology, Delhi, India, and the Ph.D. degree in electrical engineering from Stanford University, Stanford, CA, in 1998.

After completing the Ph.D. degree, he was a Principal Member Technical Staff in the Information Sciences Center, AT\&T Shannon Laboratories, Florham Park, NJ. After that, he was on the faculty at the School of Computer and Communication Sciences, EPFL, where he directed the Laboratory for Information and Communication Systems (LICOS). He is currently a Professor in the Department of Electrical Engineering, University of California, Los Angeles. His research interests include wireless communications networks, information theory, network data compression, and network algorithms.

Dr. Diggavi is a recipient of the 2006 IEEE Donald Fink prize paper award, the 2005 IEEE Vehicular Technology Conference best paper award, and the Okawa foundation research award. He is currently an editor for ACM/IEEE TRANSACTIONS ON NETWORKING and the IEEE TRANSACTIONS ON INFORMATION THEORY. He has eight issued patents.
Christina Fragouli (M'08) received the B.S. degree in electrical engineering from the National Technical University of Athens, Athens, Greece, in 1996, and the M.Sc. and Ph.D. degrees in electrical engineering from the University of California, Los Angeles, in 1998 and 2000, respectively.

She is a tenure-track Assistant Professor with the School of Computer and Communication Sciences, Ecole Polytechnique Fédérale de Lausanne (EPFL), Lausanne, Switzerland. She has been with the Information Sciences Center, AT\&T Shannon Labs, Florham Park, NJ, and the National University of Athens. She also visited Bell Laboratories, Murray Hill, NJ, and DIMACS, Rutgers University. From 2006 to 2007, she was an FNS Assistant Professor with the School of Computer and Communication Sciences, EPFL, Switzerland. Her research interests are in network information flow theory and algorithms, network coding, and connections between communications and computer science.

Dr. Fragouli served as an editor for IEEE COMMUNICATIONS LETTERS. She is currently serving as an editor for IEEE TRANSACTIONS ON INFORMATION THEORY, IEEE TRANSACTIONS ON COMMUNICATIONS, Elsevier Computer Communications, and the IEEE TRANSACTIONS ON MOBILE COMPUTING. She was the Technical Co-Chair for the 2009 Network Coding Symposium in Lausanne, Switzerland, and has served on program committees of several conferences. She received the Fulbright Fellowship for her graduate studies, the Outstanding Ph.D. Student Award 2000-2001, UCLA, Electrical Engineering Department, the Zonta award 2008 in Switzerland, and the Young Investigator ERC starting grant in 2009.

David N. C. Tse (M'96-SM'907-F'09) received the B.A.Sc. degree in systems design engineering from the University of Waterloo, Waterloo, ON, Canada, in 1989, and the M.S. and Ph.D. degrees in electrical engineering from the Massachusetts Institute of Technology, Cambridge, in 1991 and 1994, respectively.

From 1994 to 1995, he was a postdoctoral member of technical staff at AT \& T Bell Laboratories. Since 1995, he has been with the Department of Electrical Engineering and Computer Sciences, University of California at Berkeley, where he is currently a Professor.

Dr. Tse received a 1967 NSERC 4-year graduate fellowship from the government of Canada in 1989, a NSF CAREER award in 1998, the Best Paper Awards at the Infocom 1998 and Infocom 2001 conferences, the Erlang Prize in 2000 from the INFORMS Applied Probability Society, the IEEE Communications and Information Theory Society Joint Paper Award in 2001, the Information Theory Society Paper Award in 2003, and the 2009 Frederick Emmons Terman Award from the American Society for Engineering Education. He has given plenary talks at international conferences such as ICASSP in 2006, MobiCom in 2007, CISS in 2008, and ISIT in 2009. He was the Technical Program co-chair of the International Symposium on Information Theory in 2004 and was an Associate Editor of the IEEE TRANSACTIONS ON INFORMATION THEORY from 2001 to 2003. He is a coauthor, with P. Viswanath, of the text Fundamentals of Wireless Communication, which has been used in over 60 institutions around the world. 